\title{
CONFINEMENT MODELS AT FINITE TEMPERATURE AND DENSITY
}

\author{
by \\ Pok Man Lo \\ B.S. in Physics, The Chinese University of Hong Kong, 2005 \\ M.S. in Physics, University of Pittsburgh, 2007
}

Submitted to the Graduate Faculty of

Arts and Sciences in partial fulfillment

of the requirements for the degree of

Doctor of Philosophy

University of Pittsburgh

2011 


\section{UNIVERSITY OF PITTSBURGH DEPARTMENT OF PHYSICS AND ASTRONOMY}

This dissertation was presented

by

Pok Man Lo

It was defended on

August 4, 2011

and approved by

Eric Swanson, $\mathrm{PhD}$

Daniel Boyanovsky, PhD

Adam Leibovich, $\mathrm{PhD}$

Vladimir Savinov, PhD

Roy Briere, $\mathrm{PhD}$

Dissertation Director: Eric Swanson, PhD 


\title{
CONFINEMENT MODELS AT FINITE TEMPERATURE AND DENSITY
}

\author{
Pok Man Lo, PhD \\ University of Pittsburgh, 2011
}

This work presents a non-perturbative study of dynamical chiral symmetry breaking in various confinement models at finite temperature and density. The tool of choice is the Schwinger-Dyson equations. We shall discuss (i) the inadequacy of static confinement models in explaining thermal properties of QCD (ii) dynamical chiral and parity symmetry breaking of three dimensional QED with two-component fermions (iii) endemic infrared divergences in QED3 at finite temperature and the attempt to solve the system self-consistently. 


\section{TABLE OF CONTENTS}

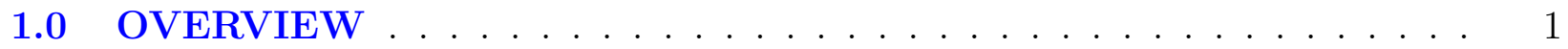

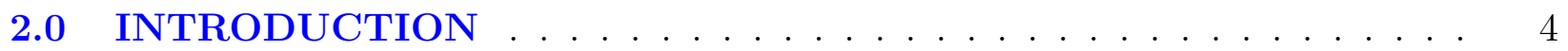

2.1 FUNCTIONAL METHOD IN QFT AND SCHWINGER-DYSON EQUA-

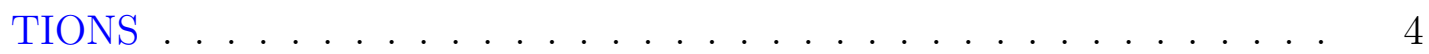

2.1.1 GENERATING FUNCTIONALS . . . . . . . . . . . . 4

2.1.2 SCHWINGER-DYSON EQUATIONS . . . . . . . . . . 6

2.2 GAUGE SYMMETRY AND WARD IDENTITY . . . . . . . . . . 8

2.2.1 MASTER EQUATIONS FOR GAUGE TRANSFORMATION . . 8

2.2.2 WARD IDENTITY FOR THE VERTEX FUNCTION $\ldots \ldots$

2.2.3 WARD IDENTITY FOR THE PHOTON PROPAGATOR . . . . 11

2.3 INTRODUCTION TO FINITE TEMPERATURE FIELD THEORY . . . 11

2.4 SYMMETRY BREAKING . . . . . . . . . . . . . . . . . . . . 13

2.4.1 CLASSICAL SYMMETRY AND NOETHER'S THEOREM . . . 13

2.4.2 CONSEQUENCE OF SYMMETRY IN QUANTUM THEORY . . 14

2.4 .3 GOLDSTONE BOSONS . . . . . . . . . . . . . . 15

2.4.3.1 NON-TRIVIAL VACUUM . . . . . . . . . . . . . 15

2.4.3.2 FORMAL DERIVATION OF THE GOLDSTONE THEO-

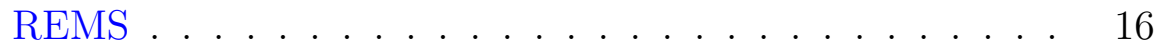

2.4.4 HIGGS MECHANISM IN ELECTROWEAK SPONTANEOUS SYMMETRY BREAKING . . . . . . . . . . . . . . . 17

2.4.5 A NOTE ON TECHNICOLOR . . . . . . . . . . . . 20

2.4.6 VECTOR AND AXIAL VECTOR SYMMETRY IN QCD . . . . 21 
2.5 CHIRAL SYMMETRY IN QCD . . . . . . . . . . . . . . 23

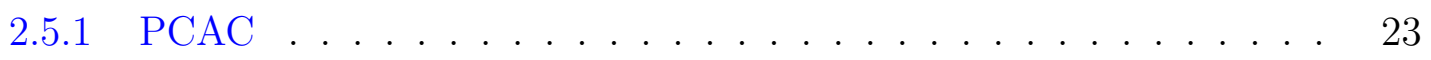

2.5.2 SOFT PION THEOREM AND GELL-MANN-OAKES-RENNER

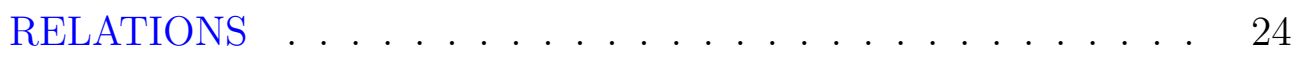

2.5.3 GOLDBERGER-TREIMAN RELATION . . . . . . . . . 26

2.6 ORDER PARAMETER, PHASE TRANSITION AND SYMMETRY BREAK-

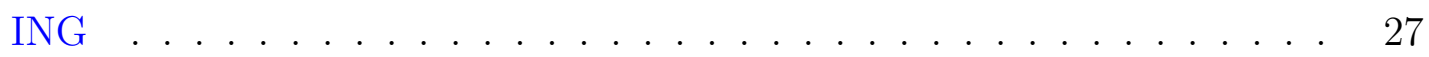

2.6.1 ORDER PARAMETER . . . . . . . . . . . . . . 27

2.6.2 PHASE TRANSITION AND SYMMETRY BREAKING . . . . 29

2.6.3 LANDAU THEORY FORMULATION OF THE CHIRAL SYMMETRY BREAKING . . . . . . . . . . . . . . . . . . 29

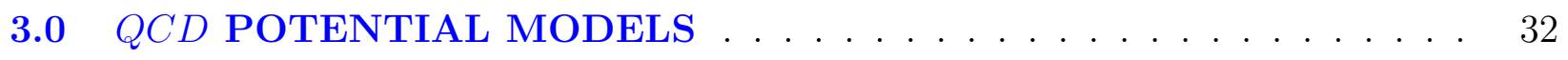

3.1 CONFINEMENT MODELS . . . . . . . . . . . . . . 33

3.1 .1 LINEAR AND CONTACT MODELS . . . . . . . . . . . . 33

3.1.2 THE GAP EQUATION AT ZERO TEMPERATURE . . . . . . 35

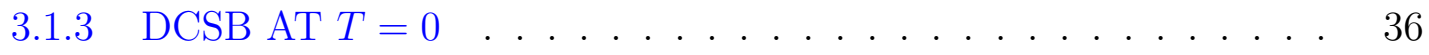

3.1.3.1 THE CONDENSATE . . . . . . . . . . . . 36

3.1.3.2 CONTACT MODEL AT T $=0 \ldots \ldots \ldots \ldots$

3.1.3.3 LINEAR MODEL AT $T=0 \ldots \ldots \ldots$

3.2 FINITE TEMPERATURE . . . . . . . . . . . . . . . . . . . . . 39

3.2.1 GAP EQUATIONS AT FINITE TEMPERATURE . . . . . . . 39

3.2.2 INFRARED DIVERGENCE IN THE GAP EQUATIONS $\ldots . .41$

3.2 .3 POLARIZATION EFFECTS . . . . . . . . . . . . . . . 41

3.2.3.1 RING APPROXIMATION . . . . . . . . . . . 41

3.2.3.2 EXPRESSION OF THE RING . . . . . . . . . . 43

3.3 PHASE STRUCTURE OF QCD . . . . . . . . . . . . . . . 47

3.4 PHASE STRUCTURE OF POTENTIAL MODELS . . . . . . . . . . 48

3.4.1 RESULTS: CONTACT POTENTIAL AT T $>0 \ldots \ldots \ldots$

3.4 .1 .1 PHASE MAP . . . . . . . . . . . . . . . . . 49 
3.4.1.2 ORDER OF PHASE TRANSITION AND THE EXISTENCE OF MULTIPLE SOLUTIONS . . . . . . . . . . 51

3.4.1.3 PHASE MAPPING BY DIFFERENTIAL EQUATION . . 53

3.4.1.4 THE EFFECT OF $\lambda \ldots \ldots \ldots \ldots \ldots$

3.4.1.5 EFFECTS OF RING . . . . . . . . . . . 56

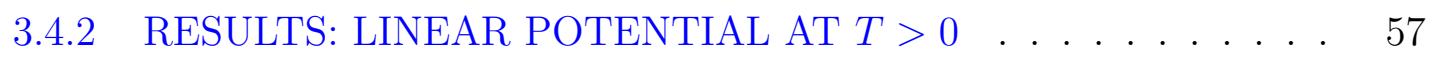

3.4.2.1 PHASE MAP AND PHASE DIAGRAM . . . . . . . 57

3.4 .2 .2 EFFECTS OF RING . . . . . . . . . . . . . 59

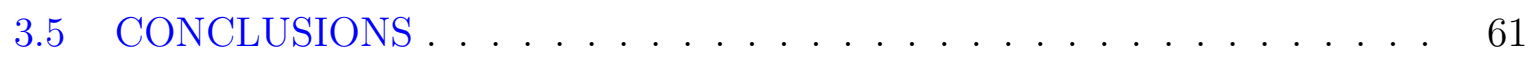

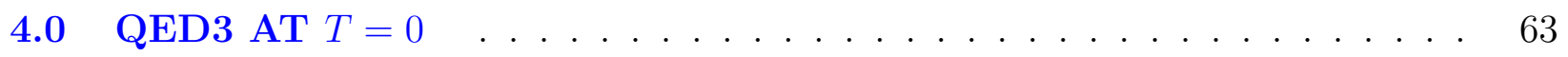

4.1 LAGRANGIAN . . . . . . . . . . . . . . . . . . 64

4.2 2-SPINOR FORMALISM . . . . . . . . . . . . . . . . . . 64

4.3 SCHWINGER-DYSON EQUATIONS . . . . . . . . . . . . 66

4.3.1 WARD IDENTITY AND TRUNCATION . . . . . . . . 66

4.3.2 GAP EQUATION FOR THE FERMIONS . . . . . . . . . . . 68

4.3 .3 PHOTON PROPAGATOR . . . . . . . . . . . . . . 70

4.3 .3 .1 INTRODUCTION . . . . . . . . . . . . . . 70

4.3.3.2 TENSOR PROPERTIES . . . . . . . . . . . . 71

4.4 PERTURBATIVE LIMIT OF THE POLARIZATION SCALARS . . . . 74

4.5 COLEMAN-HILL THEOREM . . . . . . . . . . . . . 76

4.6 NUMERICS AND RESULTS . . . . . . . . . . . . . . . . 78

4.6.1 STRATEGY OF SOLVING SDES . . . . . . . . . . . 78

4.6.2 PARITY SYMMETRY BREAKING _ . . . . . . . . . . . . 79

4.6 .3 CHIRAL SYMMETRY BREAKING . . . . . . . . . . . 81

4.6.3.1 NOTES ON CHIRAL PHASE RESTORATION . . . . . . 85

4.6.4 THE COLEMAN-HILL CONSTRAINT . . . . . . . . . . . 88

4.6 .5 NUMERICAL ASIDES . . . . . . . . . . . . . . . . 88

4.6.5.1 INTERPOLATOR . . . . . . . . . . . . . 88

4.6.5.2 NUMERICAL METHODS . . . . . . . . . . . . . . . 90

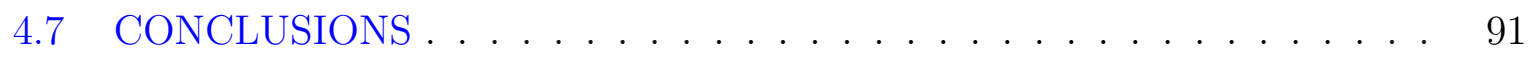


5.0 QED3 AT FINITE TEMPERATURE AND DENSITY . . . . . . . . 93

5.1 TENSOR STRUCTURES AND POLARIZATION SCALARS IN FINITE

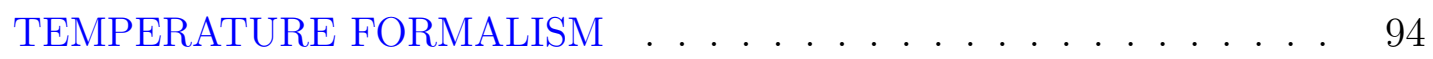

5.2 THE PHOTON PROPAGATOR . . . . . . . . . . . . . . . . 96

5.2.1 QED3 IN 4-SPINOR REPRESENTATION . . . . . . . . . . . 99

5.2 .2 QED3 IN 2-SPINOR REPRESENTATION . . . . . . . . . . 100

5.3 ENDEMIC INFRARED DIVERGENCE IN QED3 . . . . . . . . . . 101

5.3.1 PERTURBATIVE STUDY OF THE POLARIZATION TENSOR 102

5.3.2 ABSENCE OF MAGNETIC MASS IN QED3 . . . . . . . 107

5.3 .3 ENDEMIC INFRARED DIVERGENCE . . . . . . . . . . 108

5.4 SCHWINGER-DYSON EQUATIONS . . . . . . . . . . . . . . . . 111

5.4 .1 THE GAP EQUATIONS . . . . . . . . . . . . . . . . 111

5.4.1.1 FERMIONS . . . . . . . . . . . . . . . . . . . . . 111

$5.4 .2 \quad$ PHOTONS . . . . . . . . . . . . . . . . . . 113

5.4.3 A NOTE FOR ZERO TEMPERATURE AND DENSITY . . . . . 118

5.5 ASPECTS OF ELECTRIC SCREENING . . . . . . . . . . . . . . 119

5.5 .1 DEFINITION . . . . . . . . . . . . . . . . . . . 119

5.6 NUMERICS AND RESULTS . . . . . . . . . . . . . . . . . . 122

5.6 .1 QUENCHED CASE . . . . . . . . . . . . . . . . . . 123

5.6 .2 UNQUENCHED CASE . . . . . . . . . . . . . . . . . . 124

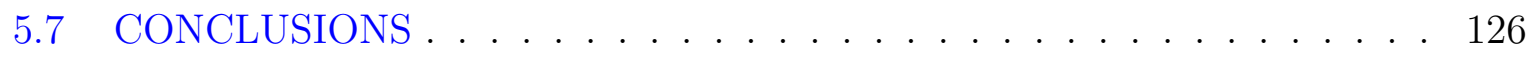

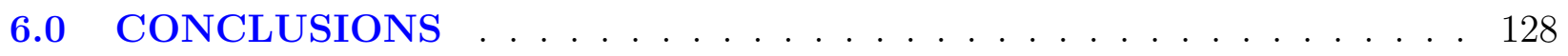

APPENDIX A. CONVENTIONS . . . . . . . . . . . . . . . . . 131

A.1 METRICS AND GAMMA MATRICES . . . . . . . . . . . . . . 131

A.2 EUCLIDEAN SPACE CONVENTIONS . . . . . . . . . . . . . . 133

APPENDIX B. FUNCTIONAL METHODS IN QFT . . . . . . . . . . 134

B.1 CENTRAL IDENTITIES OF QFT . . . . . . . . . . . . . . 135

B.2 FUNCTIONAL APPROACH TO FREE FIELD THEORY . . . . . . . 136

B.2.1 SCALARS . . . . . . . . . . . . . . . 136

B.2.2 FERMIONS . . . . . . . . . . . . . . . . . . . . 138 
B.2.3 GAUGE FIELDS . . . . . . . . . . . . . . . . . . . . . . . . 140

B.3 GAUGE FIXING FOR YANG-MILLS THEORY . . . . . . . . . . . . . . 141

B.3.1 COULOMB GAUGE IN QED . . . . . . . . . . . . . . 143

B.3.2 COULOMB GAUGE IN QCD . . . . . . . . . . . . . 146

APPENDIX C. NOTES ON PERFORMING MATSUBARA SUM . . . 150

APPENDIX D. GAP EQUATIONS FOR QED3 AT $T=0 \ldots \ldots \ldots \ldots$

D.1 BALL-CHU VERTEX . . . . . . . . . . . . . . . . . . . . . 156

D.2 CURTIS-PENNINGTON VERTEX . . . . . . . . . . . . . . . . . . 161

BIBLIOGRAPHY . . . . . . . . . . . . . . . . . . . . . . . . . 169 


\section{LIST OF TABLES}

1 Multiplication relations for the set of tensors. The six transverse tensors form a closed space. . . . . . . . . . . . . . . . . . . . . . . . . . . 95 


\section{LIST OF FIGURES}

1 Phase diagram for a ferromagnet. The surface of the equation of state is defined by the magnetization $M$ (the order parameter), the external magnetic field $B$ and the temperature $T \ldots \ldots \ldots \ldots \ldots$

2 Schwinger-Dyson equation for the full fermion propagator. The instantaneous potential is represented by the vertical gluon line. . . . . . . . . . . . . . 35

3 Solution to the gap equation for contact potential at $\lambda=3 \lambda_{c}$. At $m=0$, non-trivial solution exists $(M=1.9)$. All quantities in units of $\Lambda$. . . . . 38

4 Solution to the gap equation for linear potential. All quantities in units of $\mathrm{GeV}$. Current mass $m$ is set to zero. . . . . . . . . . . . . . . . 39

5 Gap equations with polarization insertions. . . . . . . . . . . . . . 42

6 The origin of ring contribution in the gap equations: the four-point function. For comparison, see Figure 2. . . . . . . . . . . . . . . . . . . 43

7 The schematic phase diagram of QCD with 2 massless quarks. The dashed line (red) represents second order transition, the solid line (blue) is the first order transition. The point $\mathrm{P}$ is tricritical. $T_{c} \approx 0.17 \mathrm{GeV}$ from lattice calculations.

8 Dynamical mass versus temperature and density for bare contact model. $\lambda=3 \lambda_{c} 50$

9 Typical $\mathcal{F}[M]$ function for small $\mu$. The graph shows that the phase transition is second order in nature. . . . . . . . . . . . . . . . . . . 52

10 Typical $\mathcal{F}[M]$ function for large $\mu$. The diagram shows that the phase transition is first order in nature . . . . . . . . . . . . . . . . . 
11 The phase diagram for bare contact interaction at various couplings. $\mu^{\star}$ signifies the point at which the order of phase transition goes from second order to first order. . . . . . . . . . . . . . . . . . . .

12 Dynamical mass versus temperature and density for the contact model with: (i) bare potential (red) (ii) static, zero momentum ring (green) (iii) static, momentum dependent ring (blue). For all cases, $\lambda=1.5 \lambda_{c} \ldots \ldots$. . . .

13 Dynamical mass versus temperature and density for bare linear model (AAL). All quantities in GeV. . . . . . . . . . . . . . . . .

14 Phase diagram for bare linear model (AAL). The red and green dots correspond to second and first order phase transition respectively. All quantities in GeV.

15 Dynamical mass versus temperature and density for linear potential with matter part of ring function under static, long wavelength limit. All quantities in $\mathrm{GeV}$.

16 Schwinger-Dyson equations for fermions. . . . . . . . . . . . . 68

17 Schwinger-Dyson equations for the photon. . . . . . . . . . . . . . 70

18 Two-loop corrections to $\mathcal{F}[k]$. By Coleman-Hill theorem, the sum of their contributions to $\mathcal{F}[0]$ should be exactly zero. . . . . . . . . . . . . . . 77

19 Parity-breaking solution vs $\eta$. Pluses are RL and $\mathrm{X}$ s are CBC. . . . . . . . 80

$20 A$ vs. $p$ for various $N_{f}(\mathrm{~L})$. These curves are obtained in the CBC truncation and correspond to $N_{f}=1,2,3,4,5$, and 5.75 from top to bottom. . . . . . 81

$21 B$ vs. $p$ for various $N_{f}(\mathrm{R})$. These curves are obtained in the CBC truncation and correspond to $N_{f}=1,2,3,4,5$, and 5.75 from top to bottom. . . . . .

22 Vacuum polarization $\left(\Pi=\frac{F}{k^{2}}\right)$ vs. momentum for various $N_{f}$. These curves are obtained in the CBC truncation and correspond to $N_{f}=1,2,3,4,5$, and 5.75 from bottom to top. . . . . . . . . . . . . . . . . .

23 The condensate for (top to bottom) BC, CP, RL, and CBC models, with fit functions (described in the text). . . . . . . . . . . . . . 84

$24 \tilde{\Pi}(0)$ vs. $N_{f}$ for various models. At $N_{f}=5$, from top to bottom, these are: $\mathrm{RL}, \mathrm{CBC}, \mathrm{CP}, \mathrm{BC} . \ldots \ldots \ldots$

25 The computation of $F$ for modeled $B, A$ under various interpolating schemes. 
26 Phase diagram for quenched QED3 in RL approximation. The phase transitions are 2 nd order in nature. $\epsilon_{I R}=0.1 \ldots \ldots \ldots \ldots \ldots$

27 Phase diagram for QED3 with static perturbative ring. The phase transitions are 2 nd order in nature. $\epsilon_{I R}=0.1 \ldots \ldots \ldots \ldots$

28 Phase diagram for QED3 in RL approximation. The phase transitions are 2nd

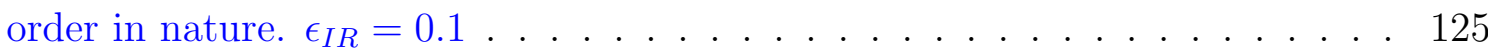

29 Illustration of gauge orbits and gauge condition: The gauge condition selects one representative element from each gauge orbit. . . . . . . . . . . . . 142 


\section{ACKNOWLEDGMENTS}

Six years is a long period of time, but it ends far too soon. In the course of my graduate studies, I am surrounded by love and support from faculty, friends and family members. It is my pleasure to express my gratitude to these individuals.

It is advised that scientific writing should be clear, concise and be purged of personal emotions. However, behind every page of cold logic is a sentimental being, reaching out to understand and be understood. Whether readers would judge this style of writing as inappropriate for a theoretical physics dissertation, or accept the balance of this work as good scientific practice, in their unamended and uncensored form, these pages must show.

Behind every good student there is a good teacher. What if the student is a slow learner, is bad at expressing himself and is constantly plagued by self-doubt? He would need a patient teacher, a caring guardian, and a supportive friend. I would like to thank my adviser, Eric Swanson, for being all three of them and in addition so much more. I am proud to be his student. Consciously and unconsciously, he has shown me how good physics is done. I appreciate all his time and efforts, his ideas and funding, his encouragement and patience during the tough times in my pursuit of $\mathrm{PhD}$. Throughout my venture into the world of non-perturbative physics, he carefully guides my study so that each transition is challenging but not intimidating. Eric also cares about my social networking in the professional circle. He has always taken time to introduce me to other people in the field. I am forever grateful for everything he has taught me, and will never stop learning from him.

This thesis could not have been accomplished without the assistance of the members of

my dissertation committee. Eric has been supportive throughout the whole process of writing and revising. I would like to thank Daniel Boyanovsky for his lectures and discussions on finite temperature field theory. He has been a very patient teacher and always explains 
physical concepts clearly. The techniques are essential for my research and I look forward to learn more from him. My sincere thanks are also due to Roy Briere, who taught a legendary course on "introduction to particle physics" in my second year of study. The final examination of that course remains among my most memorable. Roy spent a lot of extra time to discuss physics with me after class, he demonstrates how to view physics from an experimentalist's perspective. He also emphasizes the importance of understanding physicists' jokes, which I still have a lot to work on. I wish to thank Vladimir Savinov for his supporting emails and careful reading of the draft of this work, especially for the "colorful comments" he made in the text. His encouraging email put a smile on my face, while I was making the last slide of my presentation twenty minutes before the $\mathrm{PhD}$ defense. My sincere thanks also go to Adam Leibovich. Adam offers helpful advice on improving the text. I appreciate all the critical comments and the enlightening discussion in the meeting.

I would like to thank all the great staffs in the physics department. I have always enjoyed the lovely and friendly environment. I am happy to be your Bagel Boy, and I wish the replacement will do a better job than me. Special thanks go to Leyla, who, in addition to all her tedious administrative tasks, takes time in organizing department parties and preparing food for the rest of us.

I also gratefully acknowledge the institutional support that I have received while working on the project. In particular, I thank the University of Pittsburgh and the Andrew W. Mellon Foundation for supporting me with generous fellowships.

I would like to thank all my friends in Pittsburgh, who helped me to get through lots of troubles. I am especially grateful to Alice, David and Cecilia. I appreciate the time we spent together.

My warm thanks are due to professor Kwok Leung Liu in the Chinese University of Hong Kong. When I was an undergraduate in physics, he forewarned me about how careful one must be when calculating Feynman diagrams. Now every time I make a sign mistake or miss a factor of two in the process, I think of him and his lectures.

I am thankful to my friend, Jonathan Yip, in the University of Warwick, Coventry, UK. I am constantly amazed by the range of topics of our discussions: from Platonic solids to Beethoven's "Muss es sein", from the Spartans in 300 to the nasty cafeteria food in school. 
I wish him all the best in his studies.

I thank all my friends in Hong Kong for their international emotional support, which is both free of charge and professional. The twelve hours time difference between us makes it possible for me to obtain real-time support from the chat rooms, which helps me to get through the numerous long and lonely nights in the office. I really appreciate all the healthy distractions, especially those from Frankie and Ivan, though sometimes they can get a little bit too engaging! Special thanks to Mau, who lifts me up every time I fall; to Kiu, who sometimes understands me better than myself; to Carl Wong, who has been supporting me from the very beginning; and to Vivian, who has to endure my long hours of science talk, and probably needs to do so for the years to come.

I most want to thank my parents for their endless love and support. Mum and Dad, I love you so much, and I'm coming home!

I also credit my soon-to-be-two-year-old cousin, Beatrice, for inspiring and motivating me every day. Her sweet smile makes it all worthwhile. It is my hope that someday I can tell her about the wonders of this natural world. In the meantime, I will try my best to learn and explore more about it.

This thesis is dedicated to the memory of my beloved primary school teacher K.C. Jiu, who noticed when I was twelve that "this kid loves maths and science more than other subjects". Yes I did, and I still do. 


\subsection{OVERVIEW}

Dynamical chiral symmetry breaking (DCSB) provides a natural theoretical framework for the generation of masses for both the gauge bosons and the constituent fermions. In the case of low energy Quantum Chromodynamics (QCD), it successfully explains the origin of constituent-quark masses. It also establishes the connection between the partial conservation of axial-vector current and the smallness of pion mass by identifying pions as the Goldstone bosons of the hidden symmetry. The idea is also closely relevant in the building of Beyond Standard Model (BSM) physics. For example, the extended technicolor (ETC) model, aiming to solve the gauge hierarchy problem in the Standard Model, is based on the concept of dynamical mass generation.

Given the non-perturbative nature of the problem, one must use an appropriate tool to study DCSB. The tool of choice is the Schwinger-Dyson equations (SDEs), which forms an infinite tower of coupled integral equations relating the various Green's functions of the theory. With a suitable choice of truncation scheme, which is usually designed under some symmetry principles, one can extract non-perturbative information of the theory through a self-consistent calculation. Particularly, information about chiral symmetry breaking is encoded in the equation for the two-point function of the fermions, also known as the gap equation.

It is widely believed that non-perturbative phenomena like the formation of relativistic bound states from quarks and gluons (hadrons), confinement and DCSB can be adequately explained in the present framework of QCD. Lattice gauge theory, which has evolved into a powerful tool in the past decades with the advance of computing power, together with other methods such as potential models, effective field theory and DSEs, are successfully applied to QCD in the strong coupling regime. Through the combined efforts of different approaches, 
valuable insights have been gained on various important topics such as the vacuum structure, long range interaction, gluonics, quark matter and nucleon structure.

Armed with a reasonable theory of the strong interactions, it is natural to explore the properties of quark and hadronic matter under extreme conditions: high temperature and high density. Such novel environments may make their real-world appearances in

- the interior of neutron star, where the density can get several times higher than the nuclear density,

- heavy-ion collision experiments at high energies, where the conditions of both high temperature and density may be attained,

- the universe at $10^{-5} \mathrm{~s}$ after the Big Bang, where the temperature was of the same order as the nucleon rest mass.

By extending the theory to explain phenomena in a setting which is far-removed from that of its original creation, we can deepen our understanding of its theoretical structure, test for its consistency and possibly refine and improve the existing model in cases where discrepancy arises.

This work presents a non-perturbative study of dynamical chiral symmetry breaking in various confinement models at finite temperature and density. With the Schwinger-Dyson equations and the imaginary time formalism of finite temperature field theory, we study the phase structure and compute the order parameter as a function of temperature and density.

In the next chapter we start with a review on some relevant theoretical topics. Based on the functional method of quantum field theory we derive the Schwinger-Dyson equations and demonstrate how symmetries relate the various Green's functions with the example of the Ward identity in Quantum Electrodynamics (QED). The calculation scheme will be extended to finite temperature and density. We will then discuss symmetry and symmetry breaking in detail, with special focus on the case of hidden symmetry. The familiar Higgs mechanism for electroweak symmetry breaking and the extended technicolor model will be covered to illustrate both spontaneous and dynamical aspects of symmetry breaking. Lastly, we will introduce the basic notions of phase transitions such as order parameter, order of phase transition and phase diagram. The connection between phase transition and symmetry 
breaking will be clarified.

Chapter 3 focuses on in-medium chiral symmetry breaking for static potential models motivated by Coulomb gauge QCD. We shall examine the problem of infrared divergence at finite temperature in the linear confinement model, and demonstrate how summing ring diagrams resolves the issue. The phase diagrams for various models will be investigated while paying special attention to how polarization effects alter observables and the phase structure of the model systems.

In chapter 4 , we study the dynamical chiral and parity symmetry breaking of quantum electrodynamics in three dimensional spacetime (QED3). The theory is interesting in many respects. First, it serves as a model for QCD at high temperature in the large $N_{f}$ limit ${ }^{1}$. In addition, the capability of large scale dynamical masses generation makes it particularly relevant in the modeling of beyond Standard Model physics. QED3 has also been used as a model field theory for condensed matter systems such as high $T_{c}$ superconductors and graphene.

One interesting feature of the theory is that it is possible to dynamically generate a topological photon mass, which breaks both time reversal and parity symmetries. We shall examine the system with the Schwinger-Dyson equations and demonstrate the existence of a parity-violating solution. Various truncation schemes will be employed, especially the BallChu vertex, which preserves the Ward identity. The efficacy of the truncation scheme is checked with the Coleman-Hill theorem.

In chapter 5, we extend the study of QED3 to finite temperature and density. We shall see that the theory suffers from an endemic infrared divergence. A full field-theoretical calculation, with frequency and momentum dependent gauge boson propagators, will be attempted. The issue of non-perturbative renormalization associated with the electric screening mass will be addressed. We present the first self-consistent solution of the system and display the phase structure of dynamical chiral symmetry breaking.

Chapter 6 is our conclusion where we will summarize our key findings, and suggest possible directions for further study.

\footnotetext{
${ }^{1}$ At high temperature, QCD can be represented as the dimensionally reduced QCD3. Furthermore, if the number of quark flavors is large, the non-abelian nature of the theory is suppressed. These observations justify the use of QED3 as an approximation to QCD at those limits.
} 


\subsection{INTRODUCTION}

In this chapter, we review the basics. Starting with functional methods in field theory, we will derive the Schwinger-Dyson equations and demonstrate how symmetries dictate the relations among various Green's functions, as in the Ward identity in QED. The formalism will be extended to finite temperature and density. The spontaneous and dynamical aspects of symmetry breaking will be illustrated with the example of the Higgs mechanism and the ETC model. Lastly, we will introduce the basic notions of phase transitions such as order parameter and phase diagram, and highlight the connection between phase transitions and symmetry breaking. These concepts lay the foundation for our subsequent studies.

\subsection{FUNCTIONAL METHOD IN QFT AND SCHWINGER-DYSON EQUATIONS}

\subsubsection{GENERATING FUNCTIONALS}

The generating functional of a quantum field theory $[1,2,3,4,5]$ is given by

$$
Z=\int D \phi e^{i \int \mathcal{L}+j \phi}
$$

where $j(x)$ is the classical source coupled to field $\phi, \mathcal{L}$ is the Lagrangian density ${ }^{1}$.

\footnotetext{
${ }^{1}$ When there is no danger of confusion, the spacetime differential symbol $d^{N} x$ in the integral is suppressed to simplify notations.
} 
One can probe the theory by studying its response to the source. All the matrix elements required to describe physical processes can be obtained by successive functional differentiation

$$
\left\langle 0\left|T\left\{\phi_{1} \phi_{2} \ldots \phi_{n}\right\}\right| 0\right\rangle=\left.\frac{1}{Z[0]} \frac{(-i)^{n} \delta^{n}}{\delta j_{1} \ldots \delta j_{n}} Z\right|_{j \rightarrow 0}
$$

The amplitude above defines an $n$-point function, which includes both connected and disconnected contributions. For our study, it is more useful to define the generating functional for connected Green's functions

$$
W[j]=-i \ln Z
$$

Through a functional Legendre transformation, we define

$$
\Gamma[\phi]=W[j[\phi]]-\int j[\phi] \phi
$$

Note that $j[\phi]$ is treated as a functional of $\phi$ related by

$$
\phi[j]=\frac{\delta W}{\delta j},
$$

and the canonical relation

$$
j[\phi]=-\frac{\delta \Gamma}{\delta \phi}
$$

$\Gamma$ is the generating functional of the one particle irreducible (1PI) Green's function. The 1PI amplitudes are the minimal set of diagrams that generate the diversity of interactions in the QFT. The $\Gamma$ functional, together with the Schwinger-Dyson equations, provide a powerful method to extract non-perturbative information from a given theory. 


\subsubsection{SCHWINGER-DYSON EQUATIONS}

The Schwinger-Dyson equations begin with the observation that

$$
0=\int D \phi \frac{\delta}{\delta \phi} e^{i \int \mathcal{L}+j \phi}
$$

From this, one obtains the master equation

$$
\left\{\frac{\delta S}{\delta \phi}\left[\phi \rightarrow-i \frac{\delta}{\delta j}\right]+j\right\} Z[j]=0 .
$$

The corresponding equation for the generating functional of the connected Green's functions $W[j]$ is given by

$$
\left\{\frac{\delta S}{\delta \phi}\left[\phi \rightarrow\left[-i \frac{\delta}{\delta j}+\frac{\delta W}{\delta j}\right]\right]\right\} \mathbb{1}+j=0
$$

Note that to arrive at the above equation, we have used the identity

$$
\begin{aligned}
-i \frac{\delta}{\delta j}[\mathcal{F} Z[j]] & =Z\left(-i \frac{\delta \mathcal{F}}{\delta j}\right)+\mathcal{F}\left(-i \frac{\delta Z}{\delta j}\right) \\
& =e^{i W}\left\{-i \frac{\delta}{\delta j}+\frac{\delta W}{\delta j}\right\} \mathcal{F} .
\end{aligned}
$$

Effectively, any equation in the $Z$-picture of the form

$$
\mathcal{F}\left[-i \frac{\delta}{\delta j}\right] Z=0
$$

can be translated into the $W$-picture as

$$
\mathcal{F}\left[-i \frac{\delta}{\delta j}+\frac{\delta W}{\delta j}\right] \mathbb{1}=0 .
$$

Similarly, one can translate the above master equation into the $\Gamma$-picture by identifying 


$$
\begin{aligned}
& j[\phi] \longleftrightarrow-\frac{\delta \Gamma}{\delta \phi} \\
& \phi[j] \longleftrightarrow \frac{\delta W}{\delta j}
\end{aligned}
$$

and the use of the functional chain rule

$$
\begin{aligned}
\frac{\delta}{\delta j} \frac{\delta^{n} \Gamma}{\delta \phi_{1} \delta \phi_{2} \ldots \delta \phi_{n}} & =\int \frac{\delta \Phi^{\prime}}{\delta j} \frac{\delta}{\delta \Phi^{\prime}} \frac{\delta^{n} \Gamma}{\delta \phi_{1} \delta \phi_{2} \ldots \delta \phi_{n}} \\
& =\int\left(-\frac{\delta^{2} \Gamma}{\delta \phi_{j} \delta \Phi^{\prime}}\right)^{-1} \frac{\delta}{\delta \Phi^{\prime}} \Gamma^{(n)}
\end{aligned}
$$

where the second line follows from

$$
\delta=\frac{\delta j}{\delta j^{\prime}}=\int \frac{\delta \phi^{\prime}}{\delta j^{\prime}} \frac{\delta}{\delta \phi^{\prime}}\left(-\frac{\delta \Gamma}{\delta \phi_{j}}\right)=\int \frac{\delta^{2} W}{\delta j^{\prime} \delta j_{\phi^{\prime}}}\left(-\frac{\delta^{2} \Gamma}{\delta \phi^{\prime} \delta \phi_{j}}\right) .
$$

As a simple illustration, consider the scalar field theory

$$
\mathcal{L}=\frac{1}{2}\left[(\partial \phi)^{2}-m^{2} \phi^{2}\right]-V[\phi]
$$

The master equation of Schwinger-Dyson equation in $Z$-picture reads

$$
\left.\left\{-\left(\partial^{2}+m^{2}\right) \phi-\frac{\delta V}{\delta \phi}+j\right\}\right|_{\phi \rightarrow-i \frac{\delta}{\delta j}} Z=0
$$

or the corresponding $W$-picture expression

$$
-\left(\partial^{2}+m^{2}\right) \frac{\delta W}{\delta j}-\frac{\delta V}{\delta \phi}\left[\phi \rightarrow\left(-i \frac{\delta}{\delta j}+\frac{\delta W}{\delta j}\right)\right]+j=0
$$

For the case of free scalar field, performing another functional differentiation and setting the sources to zero gives the Schwinger Dyson equations for the 2-point function 


$$
\left(\partial^{2}+m^{2}\right) \frac{1}{Z[0]} \frac{\delta^{2} Z}{\delta j \delta j}=i \delta
$$

This clearly establishes the connection to the free propagator

$$
\langle 0|T\{\phi, \phi\}| 0\rangle=-\frac{1}{Z[0]} \frac{\delta^{2} Z}{\delta j \delta j}=i \Delta_{F}=i \int \frac{d^{4} p}{(2 \pi)^{4}} \frac{1}{p^{2}-m^{2}} e^{-i p \cdot x}
$$

Similarly one finds

$$
\begin{aligned}
& W^{(2)} \longleftrightarrow \frac{-1}{p^{2}-m^{2}} \\
& \Gamma^{(2)} \longleftrightarrow p^{2}-m^{2} .
\end{aligned}
$$

Generally, we interpret $W^{(2)}$ as the fully-dressed propagator and $\Gamma^{(2)}$ as the full inverse propagator.

\subsection{GAUGE SYMMETRY AND WARD IDENTITY}

\subsubsection{MASTER EQUATIONS FOR GAUGE TRANSFORMATION}

The functional approach provides a systematic way to determine the effect of a symmetry on the various N-point functions [3]. Consider a symmetry operation $\phi \rightarrow \phi+\delta \phi$

$$
\begin{aligned}
0 & =\int D \phi[\delta \mathcal{L}+j \delta \phi] e^{i \int \mathcal{L}+j \phi} \\
& =\left[\delta \mathcal{L}\left[\phi \rightarrow-i \frac{\delta}{\delta j}\right]+j \delta \phi\left[\phi \rightarrow-i \frac{\delta}{\delta j}\right]\right] Z
\end{aligned}
$$

By performing successive functional differentiation, one can derive various powerful nonperturbative restrictions on the N-point functions imposed by the symmetry. We shall illustrate how the gauge symmetry leads directly to the Ward Identity in $Q E D$. 
Consider

$$
\mathcal{L}_{Q E D}=\bar{\psi}(i \not \partial-m-g A) \psi-\frac{1}{4} F^{2}-\frac{1}{2 \xi}(\partial A)^{2},
$$

where $F_{\mu \nu}=\partial_{\mu} A_{\nu}-\partial_{\nu} A_{\mu}$ and we have included explicitly the gauge fixing term $-\frac{1}{2 \xi}(\partial A)^{2}$. Under the gauge transformation

$$
\begin{aligned}
& \psi \longrightarrow e^{-i g \alpha} \psi \approx \psi-i g \alpha \psi \\
& \bar{\psi} \longrightarrow \bar{\psi} e^{+i g \alpha} \approx \bar{\psi}+\bar{\psi} i g \alpha \\
& A \longrightarrow A+\partial \alpha
\end{aligned}
$$

we obtain

$$
\delta \mathcal{L}=-\frac{1}{\xi}\left(\partial_{\mu} A^{\mu}\right) \partial^{2} \alpha
$$

As expected, the gauge fixing term is the only term that violates gauge invariance explicitly.

To study the effect of gauge symmetry in the functional approach, consider the generating functional:

$$
Z=\int D \bar{\psi} D \psi D A e^{i \int \mathcal{L}_{Q E D}+\bar{\psi} \eta+\bar{\eta} \psi+j \cdot A}
$$

where we have introduced the Grassmann valued sources $\bar{\eta}$ and $\eta$ for the lepton fields and $j^{\mu}$ for the photon field.

One obtains the master equation for the gauge symmetry transformation

$$
0=\int D \bar{\psi} D \psi D A\left\{\frac{-1}{\xi} \partial^{2}\left(\partial_{\mu} A^{\mu}\right) \alpha+\bar{\eta}(-i g \alpha \psi)+\bar{\psi}(i g \alpha \eta)+j_{\mu} \partial^{\mu} \alpha\right\} e^{i \int \mathcal{L}_{Q E D}+\bar{\psi} \eta+\bar{\eta} \psi+j \cdot A}
$$

With the canonical relations 


$$
\begin{gathered}
\bar{\eta} \longrightarrow \frac{\delta \Gamma}{\delta \psi} \\
\eta \longrightarrow-\frac{\delta \Gamma}{\delta \bar{\psi}} \\
j \longrightarrow-\frac{\delta \Gamma}{\delta A},
\end{gathered}
$$

one obtains the equivalent master equation for gauge symmetry in $\Gamma$-picture at spacetime point $z$

$$
\frac{-1}{\xi} \partial^{2}\left(\partial_{\mu} A_{z}^{\mu}\right)+(-i g)\left(\frac{\delta \Gamma}{\delta \psi_{z}} \psi_{z}+\bar{\psi}_{z}\left(-\frac{\delta \Gamma}{\delta \bar{\psi}_{z}}\right)\right)=\partial_{z}^{\mu}\left(-\frac{\delta \Gamma}{\delta A_{z}^{\mu}}\right) .
$$

\subsubsection{WARD IDENTITY FOR THE VERTEX FUNCTION}

First, we investigate how the gauge symmetry imposes a non-trivial restriction on the longitudinal part of the vertex function.

Performing two more functional differentiations $\left(\frac{\delta^{2}}{\delta \bar{\psi}_{x I} \psi_{y J}}\right)$ on both sides of the master equations, and taking the zero field limit gives

$$
-\partial_{z}^{\mu} \frac{\delta^{3} \Gamma}{\delta A_{z}^{\mu} \delta \bar{\psi}_{x I} \delta \psi_{y J}}=(-i g)\left(\left(-\frac{\delta^{2} \Gamma}{\delta \bar{\psi}_{x I} \delta \psi_{z J}}\right) \delta_{y z}-\left(-\frac{\delta^{2} \Gamma}{\delta \bar{\psi}_{z I} \delta \psi_{y J}}\right) \delta_{x z}\right)
$$

Introducing the momentum space one particle irreducible vertex function

$$
\Gamma\left[x_{1}, \ldots, x_{n}\right]=\int \frac{d^{4} p_{1}}{(2 \pi)^{4}} \cdots \frac{d^{4} p_{n}}{(2 \pi)^{4}} e^{-i p_{1} \cdot x_{1} \ldots-i p_{n} \cdot x_{n}}(2 \pi)^{4} \delta\left[p_{1}+p_{2} \ldots p_{n}\right] \Gamma\left[p_{1}, p_{2}, \ldots p_{n}\right],
$$

one obtains

$$
-(q-p) \cdot \Gamma_{\bar{\psi} \psi A}^{(3)}[p, q]=g\left[S_{p}^{-1}-S_{q}^{-1}\right] .
$$

The longitudinal piece of the fermion-photon vertex is related to the fermionic propagator, as dictated by gauge symmetry. 


\subsubsection{WARD IDENTITY FOR THE PHOTON PROPAGATOR}

Secondly, starting with the master equation, if we perform the functional differentiation $\frac{\delta}{\delta A_{\nu}}$ and set the fields to zero, one obtains

$$
\frac{-1}{\xi} \partial^{2}\left(\partial_{\mu} \delta g^{\mu \nu}\right)=\partial_{z}^{\mu}\left(-\frac{\delta^{2} \Gamma}{\delta A^{\mu} \delta A_{\nu}}\right)
$$

The gauge symmetry prevents the longitudinal part of the photon propagator from receiving any contribution from the interaction.

\subsection{INTRODUCTION TO FINITE TEMPERATURE FIELD THEORY}

In finite temperature field theory $[6,7]$, one is concerned with the calculation of the thermal average of an observable. The partition function is defined as

$$
Z=\operatorname{Tr}\left[e^{-\beta H}\right]
$$

The imaginary time formalism allows one to write down the functional integral representation of the partition function analogous to that for the generating function at zero temperature, only that the time component is analytically continued to imaginary time $\tau=i t$ with restricted range $\left[0, \beta=\frac{1}{k T}\right]$.

$$
\begin{aligned}
& Z=\int_{B . C} D \phi e^{-S} \\
& S=\int_{0}^{\beta} d \tau \int d^{3} x \mathcal{L}[\phi[\tau, \vec{x}], \partial \phi[\tau, \vec{x}]] .
\end{aligned}
$$

The boundary condition is periodic for bosonic fields: $\phi[0, \vec{x}]=\phi[\beta, \vec{x}]$, and anti-periodic for fermionic fields: $\psi[0, \vec{x}]=-\psi[\beta, \vec{x}]$. This suggests the following Fourier expansion for the fields: 


$$
\begin{aligned}
& \phi[\tau, \vec{x}]=\frac{1}{\beta} \sum_{\omega_{n}=\frac{2 n \pi}{\beta}} \int \frac{d^{3} k}{(2 \pi)^{3}} e^{-i \omega_{n} \tau+i \vec{k} \cdot \vec{x}} \phi\left[\omega_{n}, \vec{k}\right] \\
& \psi[\tau, \vec{x}]=\frac{1}{\beta} \sum_{\omega_{n}=\frac{(2 n+1) \pi}{\beta}} \int \frac{d^{3} k}{(2 \pi)^{3}} e^{-i \omega_{n} \tau+i \vec{k} \cdot \vec{x}} \psi\left[\omega_{n}, \vec{k}\right]
\end{aligned}
$$

The thermal average of any operator $\mathcal{O}$ is defined as

$$
\langle\langle\mathcal{O}\rangle\rangle=\frac{\operatorname{Tr}\left[\mathcal{O} e^{-\beta H}\right]}{\operatorname{Tr}\left[e^{-\beta H}\right]} .
$$

The various functional techniques at zero temperature is expected to apply similarly at finite temperature field theory. Simply stated, the imaginary time formalism of finite temperature field theory amounts to replacing

$$
\begin{aligned}
\int d k^{0} & \rightarrow i \frac{1}{\beta} \sum_{\omega_{n}} \\
k^{0} & \rightarrow i \omega_{n}
\end{aligned}
$$

with $\omega_{n}$ being $\frac{2 n \pi}{\beta}$ or $\frac{(2 n+1) \pi}{\beta}$, depending on the statistics of the particle, the Matsubara sum then provides the required thermodynamics.

We can illustrate the above procedure using the case of free scalar field theory. The propagator at zero temperature is

$$
\begin{aligned}
\langle 0|T\{\phi, \phi\}| 0\rangle & =\int \frac{d^{4} k}{(2 \pi)^{4}} \frac{i}{k^{2}-m^{2}+i \epsilon} e^{-i k \cdot x} \\
& =\int \frac{d^{3} k}{(2 \pi)^{3}} \frac{1}{2 E[\vec{k}]} e^{-i(E[\vec{k}] t-\vec{k} \cdot \vec{x})}
\end{aligned}
$$

where $E[\vec{k}]=\sqrt{\vec{k}^{2}+m^{2}}$, and we have assumed $t>0$ when evaluating the $k^{0}$ integral analytically.

In the imaginary time formalism, the corresponding finite temperature expression reads $(\tau=i t)$ 


$$
\begin{aligned}
\langle\langle T\{\phi, \phi\}\rangle\rangle & =\frac{i}{\beta} \sum_{\omega_{n}=\frac{2 n \pi}{\beta}} \int \frac{d^{3} k}{(2 \pi)^{3}} \frac{-i}{\omega_{n}^{2}+\vec{k}^{2}+m^{2}} e^{-i \omega_{n} \tau+i \vec{k} \cdot \vec{x}} \\
& =\int \frac{d^{3} k}{(2 \pi)^{3}} \frac{1}{2 E[\vec{k}]}\left[n e^{-E[\vec{k}] \tau}+(1+n) e^{E[\vec{k}] \tau}\right] e^{i \vec{k} \cdot \vec{x}}
\end{aligned}
$$

where $n=\frac{1}{e^{\beta E[\vec{k}]}-1}$ is the expected thermal weight function. Notice how the two expressions agree at the zero temperature limit $^{2}$.

In perturbation theory, the various Matsubara sums arise can usually be computed analytically by means of residues theorem. An outline of the technique including the case for finite temperature and density is presented in the Appendix C.

\subsection{SYMMETRY BREAKING}

\subsubsection{CLASSICAL SYMMETRY AND NOETHER'S THEOREM}

In a classical field theory, if a certain field transformation leaves the action unchanged, one can associate a conserved Noether current with the corresponding symmetry:

$$
\begin{gathered}
\phi \longrightarrow e^{-i T^{a} \alpha^{a}} \phi \\
\frac{\delta \mathcal{L}}{\delta \alpha^{a}}=0 \longrightarrow \partial_{\mu} \frac{\delta \mathcal{L}}{\delta \partial_{\mu} \alpha^{a}}=0 .
\end{gathered}
$$

The Noether current can be identified:

$$
j_{a}^{\mu}=\frac{\delta \mathcal{L}}{\delta \partial_{\mu} \alpha^{a}} .
$$

With this, one can form the charge

\footnotetext{
${ }^{2}$ The final expression of the Matsubara sum depends crucially on the sign of $\tau$. The result shown here is for $\tau<0$. The relevant details can be found in Appendix C.
} 


$$
Q=\int d^{3} x j^{0}
$$

which is time independent, i.e conserved

$$
\frac{d Q}{d t}=0
$$

\subsubsection{CONSEQUENCE OF SYMMETRY IN QUANTUM THEORY}

Identifying the Noether current is only the first step towards understanding the consequence of the symmetry in a quantum theory. Here we give a list of possible scenarios of how the symmetry is actually realized in the quantum field theory:

- The symmetry remains exact at the quantum level.

Example: $U(1)_{E M}$ in QED.

- An anomaly induced at the quantum level breaks the symmetry explicitly. Example: axial $U(1)_{A}$.

- The symmetry is only approximate, and is explicitly broken by terms in the Lagrangian.

Example: the mass term $-\bar{\psi} M \psi$, which is not invariant under the axial vector transformation

$\psi \rightarrow e^{-i \alpha^{a} T^{a} \gamma_{5}} \psi$

where $T^{a}$ 's are the generator of the $S U\left(N_{f}\right)$ flavor transformation.

- The symmetry is hidden.

This can be further divided into the following two cases:

- Spontaneous breaking.

Typically it involves a scalar field taking on a non-trivial vacuum expectation value, this is the case for the Higgs mechanism for electroweak symmetry breaking. 
- Dynamical breaking.

The dynamics itself generate the breaking without the need for a scalar particle, the effect is usually not visible in perturbation theory. This is the case for $S U\left(N_{f}\right)_{A}$ axial vector transformation in QCD

In both the spontaneous and dynamical symmetry breaking, the symmetry is actually hidden instead of broken, in the sense that the vacuum does not respect the symmetry while the Lagrangian does, this leads to the peculiar situation where the symmetry is not visible in the mass spectrum of symmetry partners while the corresponding Noether current remains conserved. The next few sections will be devoted to examining the various interesting features of a hidden symmetry in a quantum theory.

\subsubsection{GOLDSTONE BOSONS}

2.4.3.1 NON-TRIVIAL VACUUM Recall the charge of the symmetry transformation is formed by

$$
Q=\int d^{3} x j^{0}
$$

which satisfies $\frac{d Q}{d t}=0$ and hence the commutation relation

$$
[Q, H]=0
$$

Usually the vacuum is invariant under the unitary transformation $e^{-i \alpha Q}|0\rangle=|0\rangle$, meaning

$$
Q|0\rangle=0
$$

This leads to the normal degeneracy in the mass spectrum for the eigenstates of the symmetry partners, for example, $M_{N} \approx M_{P}$ is expected as the neutron and protons are related by the isospin transformation.

In the case of hidden symmetry, on the contrary, we have 


$$
Q|0\rangle \neq 0
$$

hence, we have $|\alpha\rangle=e^{-i \alpha Q}|0\rangle \neq|0\rangle$. Since $[Q, H]=0$, it is clear that $Q|0\rangle$ has the same energy as the vacuum.

In some sense, $|\alpha\rangle$ represents a kind of excitation that does not cost energy to create, as we shall see. The massless excitations are the Goldstone bosons which carry the same quantum number as $Q$.

\subsubsection{FORMAL DERIVATION OF THE GOLDSTONE THEOREMS Con-}

sider $\mathcal{L}[\vec{\phi}, \partial \vec{\phi}]$ and a transformation $\delta \vec{\phi} \rightarrow \vec{\Delta}[\vec{\phi}]$ such that $\delta \mathcal{L} \rightarrow 0$, in the functional formalism, it means

$$
\int D \vec{\phi} \vec{j} \cdot \vec{\Delta}[\vec{\phi}] e^{i \int \mathcal{L}+\vec{j} \cdot \vec{\phi}}=0 .
$$

In terms of the 1PI generating functional ( $\Gamma$-picture),

$$
-\frac{\delta \Gamma}{\delta \vec{\phi}} \cdot \vec{\Delta}[\vec{\phi}]=0
$$

Taking one more functional derivative with respect to the field $\vec{\phi}$, and noting $\frac{\delta \Gamma}{\delta \vec{\phi}}=0$ as $\vec{\phi}$ reaches its vacuum expectation value $\overrightarrow{\phi_{0}}$, we arrive at the following:

$$
\int \frac{\delta^{2} \Gamma}{\delta \overrightarrow{\phi_{0}} \delta \overrightarrow{\phi_{0}}} \cdot \vec{\Delta}[\vec{\phi}]=0
$$

Recall the momentum space representation of the two point function is given by $p^{2}-m_{\phi}^{2}$, the above equation suggests that

$$
\left.\frac{\delta^{2} \Gamma}{\delta \overrightarrow{\phi_{0}} \delta \overrightarrow{\phi_{0}}}\right|_{p^{2} \rightarrow 0}=-m_{\phi}^{2}=0
$$


whenever $\vec{\Delta}[\vec{\phi}] \neq 0$, that is, the existence of a massless boson [3].

A simple illustration of the above is in the $S U(2)$ linear sigma model. Consider $\vec{\phi}=$ $\left(\begin{array}{c}\sigma \\ \vec{\pi}\end{array}\right)$ and the symmetry transformation:

$$
\begin{gathered}
\Delta[\sigma]=\epsilon_{5}^{a} \pi^{a} \\
\Delta\left[\pi^{a}\right]=-\epsilon_{5}^{a} \sigma
\end{gathered}
$$

if the $\vec{\phi}$ takes on non-trivial vacuum expectation value: $\left(\begin{array}{c}\sigma_{0} \\ \overrightarrow{0}\end{array}\right)$, then we see that

$$
\Delta\left[\pi^{a}\right] \neq 0
$$

and the corresponding two point function

$$
\left.\frac{\delta^{2} \Gamma}{\delta \overrightarrow{\pi_{i}} \delta \vec{\pi}_{j}}\right|_{p^{2} \rightarrow 0}=-m_{\pi}^{2} \delta_{i j}=0
$$

The pions are the three massless Goldstone bosons associated with the non-trivial vacuum.

\subsubsection{HIGGS MECHANISM IN ELECTROWEAK SPONTANEOUS SYM- METRY BREAKING}

We review the famous example of electroweak spontaneous symmetry breaking through Higgs mechanism.

For the $S U(2) \times U(1)$ symmetry, we introduce the bosonic gauge fields: $W_{1}, W_{2}, W_{3}, B$, and the Lagrangian in the gauge boson sector:

$$
\mathcal{L}_{G}=-\frac{1}{4} \tilde{W}^{2}-\frac{1}{4} B^{2}
$$

where 


$$
\begin{aligned}
D_{\mu} & =\partial_{\mu}+i g_{1} B_{\mu} \frac{1}{2}+i g_{2} W_{\mu}^{i} \tau^{i} \\
\tilde{W}_{\mu \nu}^{i} & =\partial_{\mu} W_{\nu}^{i}-\partial_{\nu} W_{\mu}^{i}-g_{2} \epsilon_{i j k} W_{\mu}^{j} W_{\nu}^{k} \\
B_{\mu \nu} & =\partial_{\mu} B_{\nu}-\partial_{\nu} B_{\mu} .
\end{aligned}
$$

Note that $\vec{\tau}=\frac{1}{2} \vec{\sigma}, \vec{\sigma}$ being the three Pauli's matrices. $D_{\mu}$ can be written as

$$
D=\partial+i\left(\begin{array}{cc}
\frac{1}{2} g_{1} B+\frac{1}{2} g_{2} W^{3} & \frac{1}{2} g_{2}\left(W^{1}-i W^{2}\right) \\
\frac{1}{2} g_{2}\left(W^{1}+i W^{2}\right) & \frac{1}{2} g_{1} B-\frac{1}{2} g_{2} W^{3}
\end{array}\right)
$$

A complex scalar field $\phi=\left(\begin{array}{c}\phi^{+} \\ \phi^{0}\end{array}\right)$ is also introduced, interacting with the gauge fields through the Lagrangian

$$
\mathcal{L}_{H G}=|D \phi|^{2}-V[\phi]
$$

with

$$
V[\phi]=-\mu^{2}|\phi|^{2}+\lambda\left[|\phi|^{2}\right]^{2}
$$

The symmetry is spontaneously broken as the Higgs scalar field takes on the non-trivial vacuum configuration $\langle\phi\rangle=\left(\begin{array}{c}0 \\ \frac{v}{\sqrt{2}}\end{array}\right)$, where

$$
v=\sqrt{\frac{\mu^{2}}{\lambda}}
$$

One consequence is that some gauge bosons acquire a mass. To see this, investigate the corresponding terms in the Lagrangian

$$
|D\langle\phi\rangle|^{2}=\left(\frac{v g_{2}}{2}\right)^{2} W^{+} W^{-}+\frac{1}{8} v^{2}\left(g_{1}^{2}+g_{2}^{2}\right)\left[\frac{g_{2}}{\sqrt{g_{1}^{2}+g_{2}^{2}}} W^{3}-\frac{g_{1}}{\sqrt{g_{1}^{2}+g_{2}^{2}}} B\right]^{2}
$$


where

$$
W^{ \pm}=\frac{1}{\sqrt{2}}\left[W^{1} \mp i W^{2}\right]
$$

We highlight two important observations. First, the spontaneous symmetry breaking leads to the mixing of the bosons in the form of

$$
\left[\frac{g_{2}}{\sqrt{g_{1}^{2}+g_{2}^{2}}} W^{3}-\frac{g_{1}}{\sqrt{g_{1}^{2}+g_{2}^{2}}} B\right]
$$

naturally defining the Weinberg angle

$$
\frac{g_{1}}{g_{2}}=\tan \theta_{W}
$$

and we can associate $Z^{0}$ and photon as the physical degrees of freedom:

$$
\begin{gathered}
Z^{0} \longleftrightarrow \cos \theta_{W} W^{3}-\sin \theta_{W} B \\
\gamma \longleftrightarrow \sin \theta_{W} W^{3}+\cos \theta_{W} B .
\end{gathered}
$$

Second, the spontaneous symmetry breaking generates masses for the gauge bosons (as can be read off from the Lagrangian)

$$
\begin{aligned}
M_{W} & =v \frac{g_{2}}{2} \\
M_{Z} & =\frac{1}{2} v \sqrt{g_{1}^{2}+g_{2}^{2}} \\
\frac{M_{W}}{M_{Z}} & =\cos \theta_{W} \\
M_{\gamma} & =0 .
\end{aligned}
$$

The Fermi constant $G_{F}$ is related to $g_{2}$ and $M_{W}$ through

$$
\frac{G_{F}}{\sqrt{2}}=\frac{g_{2}^{2}}{8 M_{W}^{2}} .
$$


This identifies

$$
v=\sqrt{\frac{1}{\sqrt{2} G_{F}}} \approx 246 \mathrm{GeV} .
$$

As a by-product, fermions in the Standard Model also acquire masses $f_{l} \frac{v}{\sqrt{2}}$ from the symmetry breaking, where $f_{l}$ is the Yukawa coupling for the fermions.

It is useful to compare the electroweak spontaneous $S U(2) \times U(1)$ breaking with the QCD $S U(3)_{A}$ symmetry breaking. In the latter case, no scalar particle is required, and the effect is generally not visible in perturbation theory.

\subsubsection{A NOTE ON TECHNICOLOR}

The Higgs mechanism fails to address the following issues. First, the potential for the Higgs is put in by hand, there is no natural explanation for the negative sign of the $\mu^{2}|\phi|^{2}$ term. Second, the resultant Higgs masses $M_{H}=2 \lambda v^{2}$ are unstable against radiative corrections from the much higher energy scales. This is the gauge hierarchy problem. Extreme fine tuning is required to keep the Higgs mass to be within the electroweak scale. Third, the mechanism also fails to provide a satisfactory explanation for the broad ratios of scales in the masses of Standard Model fermions [8]. An attractive alternative is a dynamical approach to the electroweak symmetry breaking.

If the electroweak gauge bosons consume Goldstone bosons from dynamical chiral symmetry breaking instead, the resulting masses would be of order $g f_{\pi}$. Basically everything in the Higgs mechanism follows except $v \rightarrow f_{\pi}$. However, since $f_{\pi} \approx 93 \mathrm{MeV}$, the resulting $M_{W}$ would be 1000 times smaller than required. Therefore one cannot obtain the correct phenomenology from QCD flavor dynamical chiral symmetry breaking.

The technicolor model adopts the same ideas from QCD to a different setting. Starting with Weinberg's model of hypercolor [9], which is a TeV-scale version of QCD, the dynamical

chiral symmetry breaking generates the hyper-Goldstone bosons that are then eaten by the $W$ 's and $Z$ electroweak bosons. However, the model suffers from the problem that there is no mechanism to generate the fermion mass and the existence of light hyper-Goldstone bosons. 
Extended technicolor, postulated by Dimopoulos and Susskind [10] solves the above problems by introducing extra gauge interactions $S U(N)_{E T C}$, which is broken at $\Lambda_{E T C}$, to raise the fermion and hyper-Goldstone boson masses. Unfortunately, ETC introduces the problem of large flavor changing neutral currents, which are strongly suppressed in nature. This implies $\Lambda_{E T C} \sim 1 \mathrm{TeV}$, forcing the fermion masses generated to be smaller than expected. A possible rescue to the situation suggested by Holdom [11] is the scenario of walking technicolor: if the technicolor coupling ran sufficiently slowly it would enhance the value of condensate while keeping the technipion decay constant stable. As illustrated above, it is $f_{\pi}$ that set the $E W$ scale and controls the masses of $W$ 's and $Z$. A comprehensive review on this topic can be found in Hill and Simmons [12].

It is clear that a more complete understanding of dynamical mass generation is essential in the path to investigate Beyond Standard Model Physics [13].

\subsubsection{VECTOR AND AXIAL VECTOR SYMMETRY IN QCD}

This work will mainly focus on the dynamical breaking of the chiral symmetry. It is useful to review the various related symmetries in QCD.

$$
\mathcal{L}_{Q C D}=\bar{\psi}(i \not \supset-M-g \not) \psi-\frac{1}{4} G^{2}
$$

with

$$
\begin{aligned}
& M=\left[\begin{array}{llll}
m_{u} & & & \\
& m_{d} & & \\
& & m_{s} & \\
& & & \ddots
\end{array}\right] \\
& {\left[T^{a}, T^{b}\right]=i f^{a b c} T^{c}} \\
& A=T^{a} A^{a} \\
& G_{\mu \nu}^{a}=\partial_{\mu} A_{\nu}^{a}-\partial_{\nu} A_{\mu}^{a}-g f^{a b c} A_{\mu}^{b} A_{\nu}^{c} .
\end{aligned}
$$


- $U(1)_{V}$

- Transformation: $\psi \rightarrow e^{-i \alpha} \psi$

- Noether current: $j_{V}^{\mu}=\bar{\psi} \gamma^{\mu} \psi$

- Conservation: $\partial_{\mu} j^{\mu}=0$

- $U(1)_{A}$

- Transformation: $\psi \rightarrow e^{-i \alpha \gamma_{5}} \psi$

- Noether current: $j_{A}^{\mu}=\bar{\psi} \gamma^{\mu} \gamma_{5} \psi$

- Conservation: broken explicitly by

mass term: $-\bar{\psi} M \psi$,

axial anomaly.

$\partial_{\mu} j_{A}^{\mu}=2 i \bar{\psi} \gamma_{5} M \psi+\frac{N_{f} \alpha_{s}}{8 \pi} F_{\mu \nu}^{a} F_{\rho \sigma}^{a} \epsilon^{\mu \nu \rho \sigma}$

- $S U\left(N_{f}\right)_{V}$

- Transformation: $\psi \rightarrow e^{-i \alpha^{a} T^{a}} \psi$ with $T^{a}$ 's being the generators satisfying $\left[T^{a}, T^{b}\right]=$ if ${ }^{a b c} T^{c}$

- Noether current: $V_{a}^{\mu}=\bar{\psi} \gamma^{\mu} T^{a} \psi$

- Conservation: broken explicitly by the mass term $-\bar{\psi} M \psi$

$\partial_{\mu} V_{a}^{\mu}=-i \bar{\psi}\left[T^{a}, M\right] \psi$

e.g for $S U(2)_{V}$, if $m_{u}=m_{d}$, then the vector current is conserved

- $S U\left(N_{f}\right)_{A}$

- Transformation: $\psi \rightarrow e^{-i \alpha^{a} T^{a} \gamma_{5}} \psi$

- Noether current: $A_{a}^{\mu}=\bar{\psi} \gamma^{\mu} T^{a} \gamma_{5} \psi$

- Conservation: broken explicitly by the mass term $-\bar{\psi} M \psi$

$\partial_{\mu} A_{a}^{\mu}=-i \bar{\psi}\left\{T^{a}, M\right\} \psi$

e.g for $S U(2)_{A}$, for $a=3$, we get $\partial_{\mu} A_{3}^{\mu}=2 i\left[m_{u} \bar{u} \gamma_{5} u-m_{d} \bar{d} \gamma_{5} d\right]$ only when $m_{u}, m_{d} \rightarrow 0$ we will have a conserved axial current. 


\subsection{CHIRAL SYMMETRY IN QCD}

\subsubsection{PCAC}

The axial vector symmetry in QCD is broken explicitly by the mass term in the Lagrangian. Since the current quark masses for the light flavors are small relative to the hadronic scale,

we expect the axial vector symmetry is partially conserved. It turns out the symmetry is hidden, meaning the vacuum does not realize the symmetry, while the axial vector current remains conserved to high degree.

From the Noether current $A_{a}^{\mu}=\bar{\psi} \gamma^{0} \gamma_{5} T^{a} \psi$, one can form the charge

$$
Q_{5}^{a}=\int d^{3} x \bar{\psi} \gamma^{0} \gamma_{5} T^{a} \psi
$$

for which we expect

$$
\left[Q_{5}^{a}, H\right] \approx 0
$$

while

$$
Q_{5}^{a}|0\rangle \neq 0
$$

The current algebra technique in partial conservation of the axial current (PCAC) [14] is based on the direct identification of the pion field operator with the axial vector:

$$
\partial_{\mu} A_{a}^{\mu}[x] \sim \Phi_{\pi}^{a}
$$

such that

$$
\begin{aligned}
\left\langle 0\left|\Phi_{\pi}^{a}[x]\right| \pi^{b}[p]\right\rangle & =\delta_{a b} e^{-i p \cdot x} \\
\left\langle 0\left|A_{\mu}^{a}\right| \pi^{b}[p]\right\rangle & =-i p_{\mu} f_{\pi} \delta_{a b} e^{-i p \cdot x} \\
\left\langle 0\left|\partial_{\mu} A_{a}^{\mu}\right| \pi^{b}[p]\right\rangle & =-m_{\pi}^{2} f_{\pi} \delta_{a b} e^{-i p \cdot x} .
\end{aligned}
$$


We identify

$$
\begin{aligned}
& \Phi_{\pi}^{a} \longleftrightarrow \frac{-\partial_{\mu} A_{a}^{\mu}}{f_{\pi} m_{\pi}^{2}} \\
& A_{\mu}^{a} \longleftrightarrow f_{\pi} \partial_{\mu} \Phi_{\pi}^{a} .
\end{aligned}
$$

The PCAC essentially relates the smallness of pion mass to the approximate conservation of axial current.

\subsubsection{SOFT PION THEOREM AND GELL-MANN-OAKES-RENNER RELA- TIONS}

Having identified the pion field from PCAC, one can derive the soft pion theorem, which expresses the relation between a state $|\alpha\rangle$ and the degenerate state $|\alpha \pi(q \rightarrow 0)\rangle$ due to chiral symmetry.

The proof starts with the LSZ formula [15]:

$$
\begin{aligned}
\left\langle\pi^{a}(q) \beta|\hat{\mathcal{O}}[0]| \alpha\right\rangle & =i\left[\partial^{2}+m_{\pi}^{2}\right] \int d^{4} x^{\prime} e^{i q \cdot x^{\prime}}\left\langle\beta\left|T\left\{\Phi_{\pi}^{a}, \hat{\mathcal{O}}[0]\right\}\right| \alpha\right\rangle \\
& =i\left[\partial^{2}+m_{\pi}^{2}\right] \int d^{4} x^{\prime} e^{i q \cdot x^{\prime}}\left\langle\beta\left|T\left\{\frac{-\partial_{\mu} A_{a}^{\mu}}{f_{\pi} m_{\pi}^{2}}, \hat{\mathcal{O}}[0]\right\}\right| \alpha\right\rangle \\
=i\left[-q^{2}+m_{\pi}^{2}\right] \int d^{4} x^{\prime} e^{i q \cdot x^{\prime}} \frac{-1}{m_{\pi}^{2} f_{\pi}}\left[-i q_{\mu}\left\langle\beta\left|T\left\{A^{\mu}\left[x^{\prime}\right], \hat{\mathcal{O}}[0]\right\}\right| \alpha\right\rangle-\right. & \left.\delta\left[x^{\prime 0}\right]\left[A_{x^{\prime}}^{0}, \hat{O}[0]\right]\right]
\end{aligned}
$$

at the soft momentum limit $(q \rightarrow 0)$ :

$$
\left\langle\pi^{a}(q) \beta|\hat{\mathcal{O}}[0]| \alpha\right\rangle=\frac{i}{f_{\pi}}\left\langle\beta\left|\left[Q_{5}^{a}, \hat{\mathcal{O}}[0]\right]\right| \alpha\right\rangle
$$

The Gell-Mann-Oakes-Renner relations (G.O.R) follows directly from the soft pion theorem as will be recounted here.

Start with the observation: 


$$
m_{\pi}^{2}=\langle\pi|\mathcal{H}| \pi\rangle
$$

with the soft pion theorem, one obtains

$$
m_{\pi}^{2}=\frac{-1}{f_{\pi}^{2}}\left\langle 0\left|\left[Q_{5}^{b},\left[Q_{5}^{a}, \mathcal{H}\right]\right]\right| 0\right\rangle .
$$

Noting the fact that $Q_{5}^{a}$ is the generator for the chiral transformation, for any local operator, we can write

$$
\left[Q_{5}^{a}, \mathcal{O}\right]=-i \frac{\delta}{\delta \alpha^{a}} \mathcal{O}
$$

with the corresponding symmetry transformation

$$
\psi \rightarrow e^{-i \alpha^{a} T^{a} \gamma_{5}} \psi
$$

This gives us a shortcut to perform the commutator: effecting the transformation on $\mathcal{O}$ and extract the piece that is linear with $\alpha^{a}$ !

One can then obtain, with relative ease,

$$
\begin{aligned}
{\left[Q_{5}^{a}, \mathcal{H}\right] } & =\left[Q_{5}^{a}, \bar{\psi} M \psi\right] \\
& =-\bar{\psi} \gamma_{5}\left\{T^{a}, M\right\} \psi \\
{\left[Q_{5}^{b},\left[Q_{5}^{a}, \mathcal{H}\right]\right] } & =\bar{\psi}\left\{T^{b},\left\{T^{a}, M\right\}\right\} \psi
\end{aligned}
$$

and finally reaches:

$$
m_{\pi}^{2}=-\frac{1}{f_{\pi}^{2}}\left\langle 0\left|\bar{\psi}\left\{T^{b},\left\{T^{a}, M\right\}\right\} \psi\right| 0\right\rangle .
$$

For example, the case for $S U(2)_{A}$, consider $\pi^{0}$, we have

$$
m_{\pi}^{2}=-\frac{1}{f_{\pi}^{2}}\left(m_{u}+m_{d}\right)\langle 0|\bar{q} q| 0\rangle
$$

where $\bar{q} q$ is either $\bar{u} u$ or $\bar{d} d$ 


\subsubsection{GOLDBERGER-TREIMAN RELATION}

Another application of PCAC is the derivation of Goldberger-Treiman relation [14].

Consider the axial current of the nucleon:

$$
A_{\mu N}^{a}=g \overline{\psi_{N}} \gamma_{\mu} \gamma_{5} \tau^{a} \psi_{N}
$$

where $\psi_{N}=\left(\begin{array}{l}p \\ n\end{array}\right)$ is the iso-spinor representing proton and neutron.

With PCAC, the pionic contribution to the axial current can be written as:

$$
A_{\mu \text { pion }}^{a}=f_{\pi} \partial_{\mu} \Phi_{\pi}^{a}
$$

Assuming the total axial current is given by the sum of the above, we obtain:

$$
\partial^{\mu} A_{\mu}^{a}=-f_{\pi} m_{\pi}^{2} \Phi_{\pi}^{a}=i g \overline{\psi_{N}} \gamma_{5}\left\{\tau^{a}, M\right\} \psi_{N}+f_{\pi} \partial^{2} \Phi_{\pi}^{a}
$$

In the case of identical nucleon masses $M_{N}$ :

$$
\left(\partial^{2}+m_{\pi}^{2}\right) \Phi_{\pi}^{a}=-i \frac{g}{f_{\pi}} M_{N} \overline{\psi_{N}} \gamma_{5}\left(2 \tau^{a}\right) \psi_{N}
$$

This formally identify $g_{\pi N N}=\frac{g M_{N}}{f_{\pi}}$, commonly known as the Goldberger-Treiman relation.

In application, $g=1.25$ instead of 1 due to the fact that the axial current of the nucleon is renormalized, then

$$
g_{\pi N N}=g \frac{M_{N}}{f_{\pi}} \approx 12.6
$$

compared with the experimental value extracted from pion-nucleon scattering experiments [16] 


$$
\begin{aligned}
& \frac{g_{\pi N N}^{2}}{4 \pi}=14.11 \pm 0.05(\text { statistical }) \pm 0.19(\text { systematic }) \\
\Rightarrow & g_{\pi N N}^{e x p} \approx 13.3
\end{aligned}
$$

which is in remarkably close agreement.

We see that the equation relates the quantities of weak interaction to the coupling of the strong pion nucleon interaction. The reason behind its success is due to the fact that the two phenomena are related by a symmetry, namely, the chiral symmetry.

\subsection{ORDER PARAMETER, PHASE TRANSITION AND SYMMETRY BREAKING}

\subsubsection{ORDER PARAMETER}

In a ferromagnetic system, in addition to temperature and external magnetic field, the state of the system is characterized by a thermodynamic variable that is conjugate to the external field: the magnetization. This useful variable specifies the phase of the system and is termed the order parameter $[2,17]$ :

Definition: The order parameter for a phase transition is defined as a quantity which vanishes in one phase (the disordered phase) while being non-zero in the other phase (the ordered phase)

The order parameter, temperature and the external thermodynamic field together define a surface of the equation of state of the system. Shown below is a typical graph for a ferromagnetic system. 


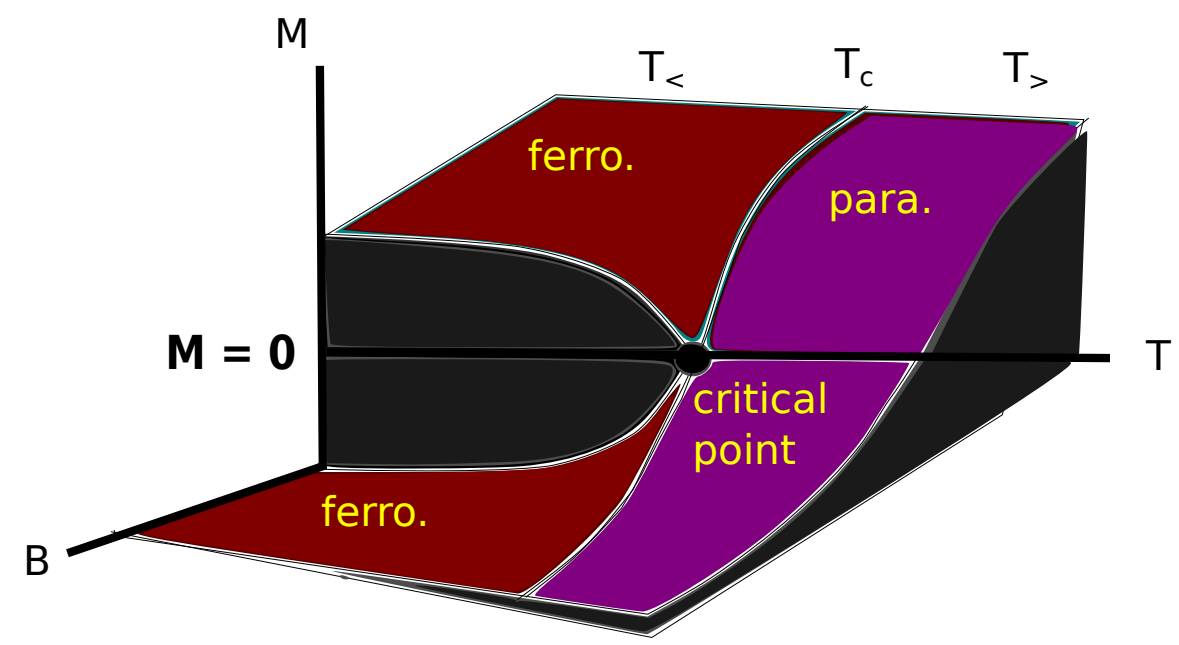

Figure 1: Phase diagram for a ferromagnet. The surface of the equation of state is defined by the magnetization $M$ (the order parameter), the external magnetic field $B$ and the temperature $T$

As the system undergoes a phase transition, the order parameter changes along the way. We call the phase transition

- first order if the change in order parameter is discontinuous,

- second order if the change is continuous.

In general, the definition of the order parameter is not unique. One can usually associate it with the thermal average (or vacuum expectation value) of an observable. Famous examples include the magnetization per spin $m$ in a ferromagnet, density difference $\rho_{\text {liquid }}-\rho_{\text {gas }}$ in the liquid-gas phase transition of a fluid, the condensate $\langle\bar{\psi} \psi\rangle$ in the chiral phase transition, etc. 


\subsubsection{PHASE TRANSITION AND SYMMETRY BREAKING}

A Phase transition usually manifests itself in the breaking of a symmetry ${ }^{3}$, for example, in the case of zero field ferromagnetic system, it involves the spontaneous breaking of the rotational symmetry: when the magnetization selects a certain direction in space. The breaking of the symmetry, in turn, is reflected in the behavior of the order parameter. The magnitude of the order parameter tells us how badly the symmetry is broken, while the sign or direction of it tells us the nature of the breaking.

In this work we will focus on the study of dynamical chiral symmetry breaking at finite temperature and density. The current mass of the fermion is the external thermodynamic field variable, while the order parameter conjugate to the field is the quark condensate. We are mainly interested in the zero current mass limit, which is analogous to the zero magnetic field limit of a ferromagnetic system. We shall plot the condensate as a function of temperature and chemical potential to study the nature of phase transition for various systems at finite temperature and density.

\subsubsection{LANDAU THEORY FORMULATION OF THE CHIRAL SYMMETRY BREAKING}

Here we make an attempt to illustrate the connection between the functional approach of the quantum field theory and the Landau formulation in studying symmetry breaking. We will restrict ourselves to consider the following Nambu-Jona-Lasinio-type model [18]:

$$
Z=\int D \bar{\psi} D \psi e^{i \int \bar{\psi}(i \not \partial-m) \psi+G\left[(\bar{\psi} \psi)^{2}+\left(\bar{\psi} i \gamma_{5} \psi\right)^{2}\right]}
$$

In the Landau formulation, the order parameter is singled out as the collective variable representing the degree of freedom of a phase transition.

Casting in the functional integral formalism:

\footnotetext{
${ }^{3}$ It is, however, not always the case. The liquid-gas phase transition, for example, is not associated with spontaneous symmetry breaking.
} 


$$
Z=e^{-\beta F[T, m]}=\int d \mu e^{-\beta \mathcal{L F}[\mu, T, m]}
$$

where $\mathcal{L} \mathcal{F}[\mu, T, m]$ is the Landau function.

The state of the system is specified by

$$
\left.\frac{\partial \mathcal{L} \mathcal{F}}{\partial \mu}\right|_{\mu \rightarrow \mu_{c}}=0
$$

with $\mu_{c}$ being the value of the order parameter of the system.

Observe that

$$
\begin{aligned}
e^{i G \int(\bar{\psi} \psi)^{2}} & =\int D \sigma e^{i \int\left[-\sigma \bar{\psi} \psi-\frac{1}{4 G} \sigma^{2}\right]} \\
e^{i G \int\left(\bar{\psi} i \gamma_{5} \psi\right)^{2}} & =\int D \pi e^{i \int\left[-\pi \bar{\psi} i \gamma_{5} \psi-\frac{1}{4 G} \pi^{2}\right]}
\end{aligned}
$$

One can rewrite the original generating functional as:

$$
\begin{aligned}
Z & =\int D \bar{\psi} \psi D \sigma D \pi e^{i \int \bar{\psi}\left(i \not \partial-m-\sigma-i \gamma_{5} \pi\right) \psi-\frac{1}{4 G}\left[\sigma^{2}+\pi^{2}\right]} \\
& =\int D \sigma D \pi e^{i\left[-i \operatorname{Tr} \ln \left[\left(i \not \partial-m-\sigma-i \gamma_{5} \pi\right)\right]-\int \frac{1}{4 G}\left[\sigma^{2}+\pi^{2}\right]\right]}
\end{aligned}
$$

Assuming only $\sigma$ has a non-vanishing vacuum expectation value $\sigma_{0}$, and identify:

$$
\mathcal{L} \mathcal{F}[\sigma, m]=-i \operatorname{Tr} \ln [(i \not \partial-m-\sigma)]-\int \frac{1}{4 G} \sigma^{2}
$$

We determine

$$
-i \operatorname{Tr} \frac{-1}{\left(i \not \partial-m-\sigma_{0}\right)}=\frac{\sigma_{0}}{2 G}
$$

In the vacuum limit: $Z \approx e^{i \mathcal{L F}\left[\sigma_{0}, m\right]}$

one gets: 


$$
-i \operatorname{Tr} \frac{-1}{\left(i \not \partial-m-\sigma_{0}\right)}=\frac{\sigma_{0}}{2 G}=-\frac{\partial}{\partial m}(-i \ln Z)=\langle\bar{\psi} \psi\rangle
$$

The same result can be obtained from the Schwinger-Dyson equation or mean field method under Hartree approximation. One can see how the condensate takes the role as an order parameter and the non-perturbative feature (the existence of $\frac{1}{G}$ factor) of the symmetry breaking. 


\section{0 $Q C D$ POTENTIAL MODELS}

In this chapter ${ }^{1}$, we will examine the in-medium chiral symmetry breaking in confining potential models of QCD. The knowledge of QCD at finite temperature and density is essential to understand physical systems such as neutron stars, the early universe and the heavy-ion collision experiments at RHIC and LHC [20, 21]. Many of the interesting features are nonperturbative, in fact, even the hope that QCD at large temperature is perturbative may be misplaced as the resulting theory maps to a confining three dimensional version of QCD. Therefore, it is important to develop non-perturbative methods for the analysis of QCD and the relating models.

We shall mainly focus on two static potential models motivated by Coulomb gauge QCD: contact and linear potential. The former is structurally similar to the Nambu-Jona-Lasinio models [18], while the latter is more physically motivated as it incorporates the phenomenology of confinement in the strong interactions. The infrared divergence problem in the linear model will be discussed, and the inclusion of polarization effects is proposed as a resolution. Schwinger-Dyson equations will be employed to extract the phase diagrams of the models; we are especially interested in how the observables and phase structures depend on the truncation scheme one chooses. Results and discussions will be presented at the end of the chapter.

\footnotetext{
${ }^{1}$ The research in this chapter is published in "Confinement models at finite temperature and density" [19].
} 


\subsection{CONFINEMENT MODELS}

\subsubsection{LINEAR AND CONTACT MODELS}

As our starting point, we shall consider the Lagrangian density

$$
\mathcal{L}[x]=\bar{\psi}_{x}(i \not \partial-m) \psi_{x}-\frac{1}{2} \int d^{4} y \delta^{a b} V_{x, y} \bar{\psi}_{x} \gamma^{0} T^{a} \psi_{x} \bar{\psi}_{y} \gamma^{0} T^{b} \psi_{y}
$$

with $\psi, \bar{\psi}$ being the fermionic fields.

The form of the Lagrangian is motivated from QCD in Coulomb gauge ${ }^{2}$, upon neglecting the transverse gluons. The potential is formally identified as the generalized Coulomb potential $[22,23]$

$$
\delta^{a b} V_{x, y} \longleftrightarrow\left\langle 0\left|\left(\vec{x} a\left|\frac{1}{\vec{\nabla} \cdot \overrightarrow{\mathcal{D}}}\left(-\vec{\nabla}^{2}\right) \frac{1}{\vec{\nabla} \cdot \overrightarrow{\mathcal{D}}}\right| \vec{y} b\right)\right| 0\right\rangle
$$

where $\mathcal{D}_{\mu}^{a c}=\partial_{\mu} \delta^{a c}-g f^{a b c} A_{\mu}^{b}$ is the adjoint covariant derivative, with $a, b, c$ being color indices.

For our study, we will consider the following two potentials

- the contact model:

$$
V[\vec{r}]=\lambda \delta[\vec{r}] \longleftrightarrow V[\vec{k}]=\lambda
$$

- the linear confinement model:

$$
V[\vec{r}]=-\frac{3}{4} b r \longleftrightarrow V[\vec{k}]=\frac{6 \pi b}{k^{4}}
$$

where $b$ is the string tension, with its phenomenological value being approximately $0.2 \mathrm{GeV}^{2}$.

\footnotetext{
${ }^{2}$ The derivation of the Lagrangian of QCD in Coulomb gauge is detailed in Appendix B.3.2.
} 
Note that for the linear potential, the Fourier transform is not well-defined:

$$
\begin{aligned}
V(r) & =\int \frac{d^{3} k}{(2 \pi)^{3}} \frac{6 \pi b}{k^{4}} e^{i k r \cos [\theta]} \\
& =\frac{3 b}{\pi} \int d k k^{2} \frac{1}{k^{4}} \frac{\sin [k r]}{k r}
\end{aligned}
$$

The integral is infrared divergent. However, if we consider

$$
\frac{d V(r)}{d r}=\frac{1}{4 \pi^{2}} \int d k d z k^{2} i k z \frac{6 \pi b}{k^{4}} e^{i k r z}
$$

The integral is finite and can be evaluated exactly as

$$
\begin{aligned}
\frac{d V(r)}{d r} & =\frac{3 b}{2 \pi} \int d k i k^{3} \frac{1}{k^{4}} \frac{2 i(-k r \cos [k r]+\sin [k r])}{k^{2} r^{2}} \\
& =-\frac{3}{4} b
\end{aligned}
$$

effectively one has

$$
\bar{V}[r]=V[r]-V[0]=-\frac{3}{4} b r
$$

It is in this context that we identify the Fourier transform pair for the linear potential. 


\subsubsection{THE GAP EQUATION AT ZERO TEMPERATURE}

Starting with the generating functional

$$
Z=\int D \bar{\psi} D \psi e^{i \int \mathcal{L}+\bar{\eta} \psi+\bar{\psi} \eta}
$$

and recall $Z=e^{i \mathcal{W}}$, we can derive the Schwinger Dyson equation for the two point function:

$$
\begin{aligned}
\frac{\delta^{2} \mathcal{W}}{\delta \bar{\eta} \delta \eta}= & \frac{1}{i \not \supset-m} \delta+ \\
& -\int d^{4} z d^{4} z^{\prime} V_{z, z^{\prime}} T^{a} T^{a} \gamma_{I J}^{0} \gamma_{I^{\prime} J^{\prime}}^{0} S_{01 I}\left(x, z^{\prime}\right)\left[\frac{\delta^{4} \mathcal{W}}{\delta \bar{\eta}_{J}\left(z^{\prime}\right) \delta \bar{\eta}_{J^{\prime}}(z) \delta \eta_{I^{\prime}}(z) \delta \eta(y)}\right. \\
& \left.-i \frac{\delta^{2} \mathcal{W}}{\delta \bar{\eta}_{J}\left(z^{\prime}\right) \delta \eta_{I^{\prime}}(z)} \frac{\delta^{2} \mathcal{W}}{\delta \bar{\eta}_{J^{\prime}}(z) \delta \eta(y)}+i \frac{\delta^{2} \mathcal{W}}{\delta \bar{\eta}_{J}\left(z^{\prime}\right) \delta \eta(y)} \frac{\delta^{2} \mathcal{W}}{\delta \bar{\eta}_{J^{\prime}}(z) \delta \eta_{I^{\prime}}(z)}\right] .
\end{aligned}
$$

The diagrammatic representation [24] reads:

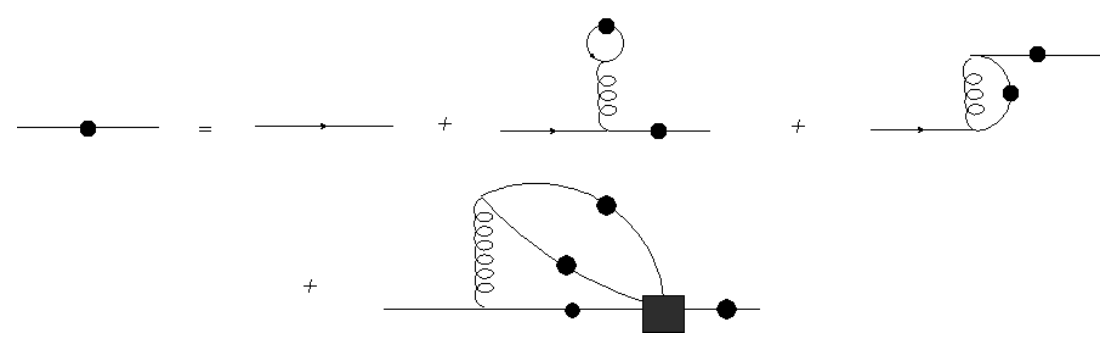

Figure 2: Schwinger-Dyson equation for the full fermion propagator. The instantaneous potential is represented by the vertical gluon line.

For our purpose, we will begin with one simple truncation scheme: neglecting the vertex correction (last diagram on the RHS). Moreover, the second diagram automatically vanishes in the present case due to the fact that $\operatorname{Tr}[T] \rightarrow 0$. Under this truncation scheme, the gap equation can be written as: (with color factor explicitly shown) 


$$
S_{12}^{-1}=S_{0}^{-1}{ }_{12}-i V_{x, y} \frac{N_{c}^{2}-1}{2 N_{c}}\left[\gamma^{0} S_{x y} \gamma^{0}\right]_{12}
$$

To solve this in a self-consistent manner, we introduce the following Ansätz for the 2 point function

$$
\frac{\delta^{2} \mathcal{W}}{\delta \bar{\eta} \delta \eta}=\int \frac{d^{4} k^{\prime}}{(2 \pi)^{4}} \frac{1}{A_{0} k_{0}^{\prime} \gamma^{0}-A \overrightarrow{k^{\prime}} \cdot \vec{\gamma}-B} e^{-i k^{\prime} \cdot(x-y)} .
$$

Substituting into the gap equation 3.11, one obtains

$$
\begin{aligned}
A_{0} & \rightarrow 1 \\
M[\vec{k}] & =\frac{m+\frac{2}{3} \int \frac{d^{3} k^{\prime}}{(2 \pi)^{3}} V_{\vec{k}-\overrightarrow{k^{\prime}} \frac{M_{\overrightarrow{k^{\prime}}}}{\epsilon_{\vec{k}^{\prime}}}}}{1+\frac{2}{3} \int \frac{d^{3} k^{\prime}}{(2 \pi)^{3}} V_{\vec{k}-\overrightarrow{k^{\prime}}} \hat{k} \cdot \hat{k}^{\prime} \frac{\left|\overrightarrow{k^{\prime}}\right|}{\epsilon_{\overrightarrow{k^{\prime}}}} \frac{1}{|\vec{k}|}} \\
\epsilon[\vec{k}] & =\sqrt{\vec{k}^{2}+M[k]^{2}}
\end{aligned}
$$

where we have introduced the constituent mass function $M(k)=\frac{B}{A}$, to be distinguished from the current mass $m$. Observe that $M[k]$ appears on both side of the equation, and should be solved self-consistently.

\subsubsection{DCSB AT $T=0$}

3.1.3.1 THE CONDENSATE The quark condensate is the order parameter for dynamical chiral symmetry breaking. With the Ansätz 3.12 for the propagator, we obtain the expression

$$
\langle\bar{\psi} \psi\rangle=\int \frac{d^{3} k^{\prime}}{(2 \pi)^{3}}(-2) \frac{M\left[\overrightarrow{k^{\prime}}\right]}{\epsilon_{\overrightarrow{k^{\prime}}}} N_{c}
$$

The key observation in DCSB is that even at $m \rightarrow 0$, the condensate can attain a non-zero vacuum expectation value due to non-perturbative effects. 
3.1.3.2 CONTACT MODEL AT $T=0$ In the case of contact potential $V[|\vec{k}|]=\lambda$, the gap equation simplifies to

$$
\begin{aligned}
M & =m+\frac{2}{3} \int^{\Lambda} \frac{d^{3} k^{\prime}}{(2 \pi)^{3}} \lambda \frac{M_{\vec{k}^{\prime}}}{\epsilon_{\vec{k}^{\prime}}} \\
& =m+\frac{1}{3 \pi^{2}} \int^{\Lambda} d k^{\prime} k^{\prime 2} \lambda \frac{M}{\epsilon^{\prime}} .
\end{aligned}
$$

Similar to the case of NJL-model [18], the theory is non-renormalizable, meaning that a regularization scheme is needed in order to define the model properly. We shall employ the three-momentum noncovariant cutoff scheme by introducing the cutoff parameter $\Lambda$, which serves as the energy scale of the problem. From now on, we shall express all quantities in units of $\Lambda$ within the discussion of contact model.

In the limit of vanishing current mass $m=0, M=0$ is always a solution, the system is said to be in Wigner mode. However, by inspecting the gap equation 3.15, one can deduce that non-trivial solution can exist if

$$
\lambda>\lambda_{c}=6 \pi^{2}
$$

where we define $\lambda_{c}$ as the critical coupling constant. With the non-trivial solution, the system is said to be in the chiral broken phase ${ }^{3}$.

The solution to the gap equation can be obtained by root-finding methods ${ }^{4}$, the case for $\lambda=3 \lambda_{c}$ is presented:

\footnotetext{
${ }^{3}$ Strictly speaking, for every system considered, one needs to check whether the non-trivial solution corresponds to a lower energy than the trivial one. Likewise, at finite temperature and density, the nontrivial solution must be shown to lead to a lower value in the thermodynamic potential [18]. It is shown to be true for a wide class of potentials [25]. For the various models we consider, we believe it is the case.

${ }^{4}$ In the case of contact potential, the constituent mass function is momentum independent. Given the current mass $m$, one simple way to obtain the corresponding constituent mass $M$ is by graphical method: plotting both sides of the gap equation 3.15 as a function of $M$ and looking for the intersection.
} 


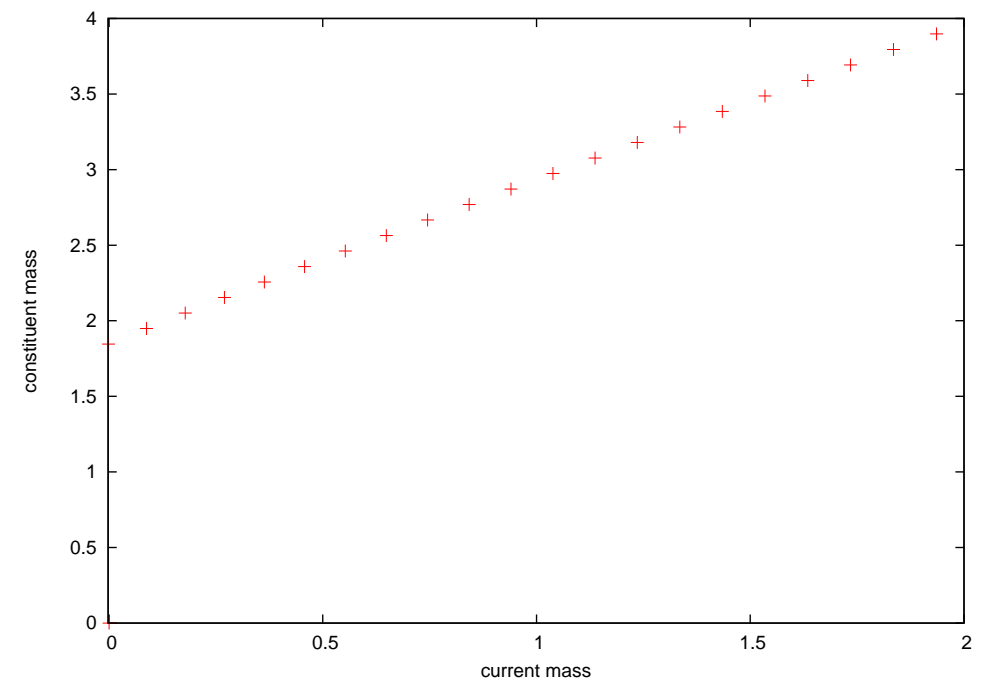

Figure 3: Solution to the gap equation for contact potential at $\lambda=3 \lambda_{c}$. At $m=0$, non-trivial solution exists $(M=1.9)$. All quantities in units of $\Lambda$.

3.1.3.3 LINEAR MODEL AT $T=0 \quad$ For the linear potential $V[|\vec{k}|]=\frac{6 \pi b}{|\vec{k}|^{4}}$, the solution to the gap equation is momentum dependent ${ }^{5}$ :

\footnotetext{
${ }^{5}$ Generally, the integral equation can be solved by minimization. Other methods like iteration and simulated annealing can be applied.
} 


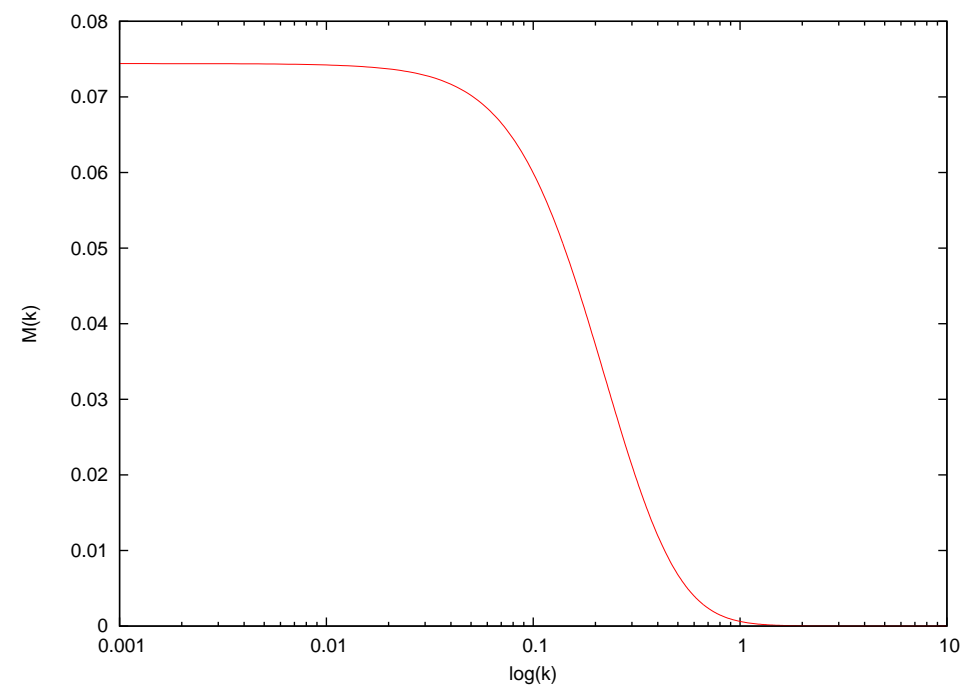

Figure 4: Solution to the gap equation for linear potential. All quantities in units of $\mathrm{GeV}$. Current mass $m$ is set to zero.

The constituent mass function $M[k]$ approaches the perturbative limit (current quark mass $m$ ) in the ultraviolet. The function is enhanced in the zero momentum limit, the value of $M(0)$ describes the dynamical breaking of the chiral symmetry. It can be used as a proxy for the quark condensate, which is the conventional order parameter of choice for DCSB.

\subsection{FINITE TEMPERATURE}

\subsubsection{GAP EQUATIONS AT FINITE TEMPERATURE}

At finite temperature and density, the interesting quantity is the thermal average instead of the vacuum expectation value. The imaginary time formalism amounts to replacing 


$$
\int d k^{0} \rightarrow i \frac{1}{\beta} \Sigma_{\omega_{n}}
$$

The Schwinger Dyson equation for the two point function becomes

$$
S_{12}^{-1}=S_{0}^{-1}{ }_{12}+\frac{1}{\beta} \sum_{\omega_{n}^{\prime}} \int \frac{d^{3} k^{\prime}}{(2 \pi)^{3}} V_{\vec{k}^{\prime}-\vec{k}} \frac{N_{c}^{2}-1}{2 N_{c}}\left[\gamma^{0} S_{\omega_{n}^{\prime}, \vec{k}^{\prime}} \gamma^{0}\right]_{12}
$$

Again, we make the Ansätz for the two point function

$$
S\left[\omega_{n}, \vec{k}\right]=\frac{1}{\left(i \omega_{n}+\mu^{\prime}\right) \gamma^{0}-A \overrightarrow{k^{\prime}} \cdot \vec{\gamma}-B} .
$$

In the case of static potential model, where $V$ does not depend explicitly on the Matsubara frequency, one can evaluate the sum analytically [6]. Performing the sum over $\omega_{n}^{\prime}=\left(n+\frac{1}{2}\right) \frac{2 \pi}{\beta}$, we arrive at the gap equations:

$$
\begin{aligned}
& B[\vec{k}]=m+\frac{2}{3} \int \frac{d^{3} k^{\prime}}{(2 \pi)^{3}} V_{\vec{k}-\overrightarrow{k^{\prime}}} \frac{B_{\overrightarrow{k^{\prime}}}}{E_{\overrightarrow{k^{\prime}}}}(1-n-\bar{n}) \\
& A[\vec{k}]=1+\frac{2}{3} \int \frac{d^{3} k^{\prime}}{(2 \pi)^{3}} V_{\vec{k}-\overrightarrow{k^{\prime}}} \hat{k} \cdot \hat{k^{\prime}} \frac{A_{\overrightarrow{k^{\prime}}}\left|\overrightarrow{k^{\prime}}\right|}{E_{\overrightarrow{k^{\prime}}}} \frac{1}{|\vec{k}|}(1-n-\bar{n}) \\
& \mu^{\prime}[\vec{k}]=\mu+\frac{2}{3} \int \frac{d^{3} k^{\prime}}{(2 \pi)^{3}} V_{\vec{k}-\overrightarrow{k^{\prime}}}(n-\bar{n})
\end{aligned}
$$

with

$$
\begin{aligned}
E_{\vec{k}} & =\sqrt{{A_{\vec{k}}^{2}}^{2}|\vec{k}|^{2}+B_{\vec{k}}^{2}} \\
n & =\frac{1}{1+e^{\beta\left[E-\mu^{\prime}\right]}} \\
\bar{n} & =\frac{1}{1+e^{\beta\left[E+\mu^{\prime}\right]}} .
\end{aligned}
$$

Note that the condensate at finite temperature is still given by

$$
\langle\bar{\psi} \psi\rangle=\int \frac{d^{3} k^{\prime}}{(2 \pi)^{3}}(-2) \frac{M\left[\overrightarrow{k^{\prime}}\right]}{\epsilon_{\overrightarrow{k^{\prime}}}} .
$$

The system of coupled integral equations must be solved simultaneously. One can generally solve it using iterative method. Other methods especially suitable for the contact model has been developed and will be discussed in section 3.4.1.2. 


\subsubsection{INFRARED DIVERGENCE IN THE GAP EQUATIONS}

The gap equations for the linear model are infrared divergent due to the $\frac{1}{k^{4}}$ momentum dependence of the potential. At zero temperature, it presents no problem since the constituent mass function $M=\frac{B}{A}$ remains divergence-free although $B$ and $A$ are separately infrared divergent. At finite temperature, as seen in the gap equation 3.20, the thermal weight functions $n$ and $\bar{n}$ depends explicitly on the divergent $A^{6}$. Thus, the infrared divergence forces them to unity, and renders the finite temperature formalism useless.

Past attempts to resolve the issue include:

- replace $E_{\vec{k}} \rightarrow E_{\vec{k}}-E_{0}$ in the thermal weight functions, as suggested by Davis and Matheson [26], who first noted the nonsensical result of the above,

- replace $E_{\vec{k}} \rightarrow \sqrt{\vec{k}^{2}+\left(\frac{B[\vec{k}]}{A[\vec{k}]}\right)^{2}}$ in the thermal weight functions, suggested by Alkofer et al. [27], we shall denote this procedure as "AAL",

- reformulate the thermodynamic trace to sum over only the color singlet states, suggested by the Orsay group [28].

None of these resolutions seem satisfactory. For the first two cases, the substitutions are completely ad hoc, although they may still be good approximations. The last approach is physically motivated, however, one expects the non-singlet states to be automatically removed by the Boltzmann factor instead of having to perform the projection explicitly.

In the next section, we demonstrate a simple resolution by suggesting an alternative truncation scheme.

\subsubsection{POLARIZATION EFFECTS}

3.2.3.1 RING APPROXIMATION Infrared divergences can normally be removed by incorporating additional physical effects (such as bremsstrahlung) or by summing additional diagrams. In the present approach, the latter can be implemented by adapting a different

\footnotetext{
${ }^{6}$ The thermal weight functions depends on generalized energy function $E[\vec{k}]=\sqrt{A^{2} \vec{k}^{2}+B^{2}}=$ $A \sqrt{\vec{k}^{2}+M[k]^{2}}$, which is infrared divergent even if the constituent mass function is infrared safe.
} 
truncation scheme. Specifically, we consider the ring approximation to the full interaction potential $^{7}$ :

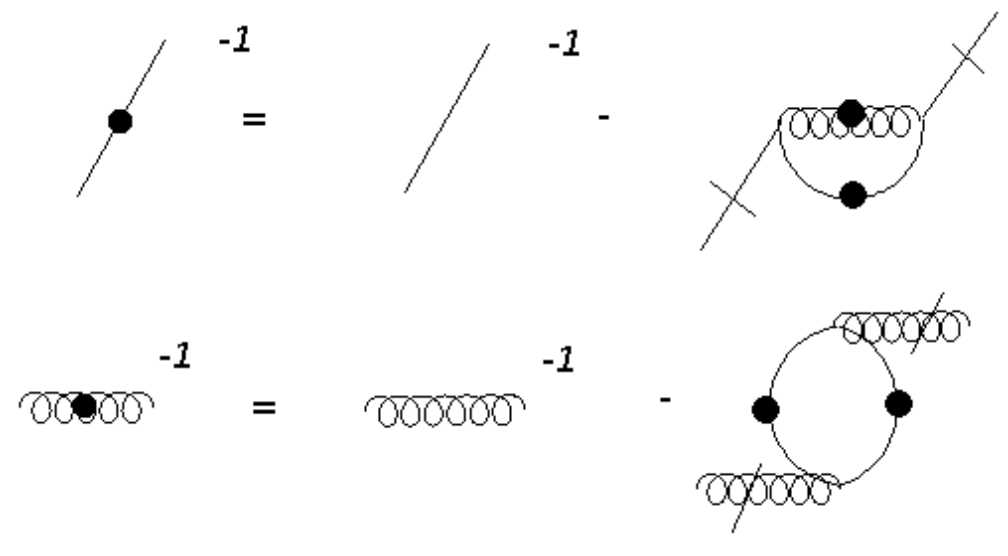

Figure 5: Gap equations with polarization insertions.

The above approximation can also be obtained by including the suitable set of diagrams in the vertex correction (the four-point function) in SDEs. Shown below is a simple demonstration of how the ring diagrams enter the gap equations through a different truncation of the 4 point functions:

\footnotetext{
${ }^{7}$ The approximation was first employed by Brueckner and Gell-Mann [29] as the resolution of infrared divergence problem in the degenerate electron gas problem.
} 


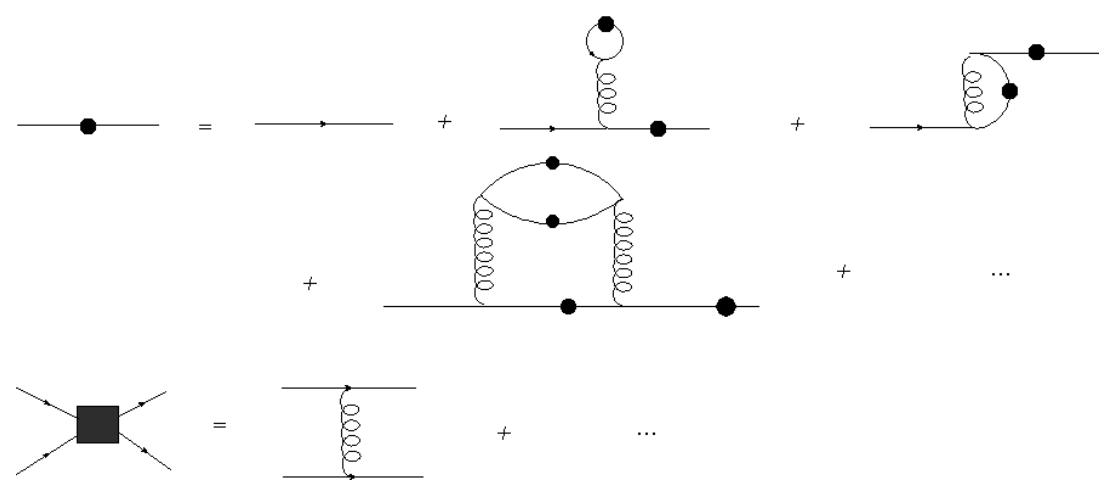

Figure 6: The origin of ring contribution in the gap equations: the four-point function. For comparison, see Figure 2.

Recall that in the present model, the potential is not dynamical, and hence can not have an Schwinger-Dyson equation in the strictest sense. The dressing of the potential in Figure 5 serves only to illustrate our truncation scheme.

3.2.3.2 EXPRESSION OF THE RING To implement the new truncation scheme, one needs to replace the bare potential with the dressed one

$$
V[k] \longrightarrow V_{\text {ring }}\left[k_{0}, \vec{k}\right]=\frac{V[\vec{k}]}{1+i \frac{1}{2} n_{f} V[\vec{k}] \Pi\left[k_{0}, \vec{k}\right]}
$$

where $\Pi[k]$ is the ring integral

$$
\Pi\left[k_{0}, \vec{k}\right]=\int \frac{d^{4} k^{\prime}}{(2 \pi)^{4}} \operatorname{Tr}\left[\gamma^{0} S_{k^{\prime}} \gamma^{0} S_{k^{\prime}-k}\right]
$$

The factor of $\frac{1}{2}$ comes from the color trace, and $n_{f}$ is the number of flavors.

In general, the dressing of the potential will make it $k_{0}$ dependent (or equivalently timedependent), which in turns substantially complicating the solution of the gap equations. However, the dominant infrared contribution to the ring potential is in the static limit: 
$k_{0} \rightarrow 0$. We shall restrict our attention only to this limit. The resulting gap equations remains the same as in equation 3.20 , except $V[\vec{k}] \rightarrow V_{\text {ring }}\left[k_{0}=0 ; \vec{k}\right]$.

At zero temperature, the ring integral is divergent and needs to be regulated. Here we record the results in the perturbative limit under various regularization schemes:

- 4D regularization

$$
\Pi=i \frac{1}{4 \pi^{2}} \int_{0}^{1} d x\left[\frac{1}{2} \Lambda_{4 D}^{2}-x(1-x) 2 \vec{k}^{2} \ln \frac{\Lambda_{4 D}^{2}}{\Delta}\right]
$$

- Dimensional regularization

$$
\Pi=i \frac{1}{4 \pi^{2}} \int_{0}^{1} d x-x(1-x) 2 \vec{k}^{2}\left[\ln \frac{\mu_{D R}}{\Delta}+\frac{2}{\epsilon}+\ln (4 \pi)-\gamma\right]
$$

- 3D regularization

$$
\Pi=-i \frac{1}{4 \pi^{2}} \int d x x(1-x) 2 \vec{k}^{2} \ln \frac{4 \Lambda_{3 D}^{2}}{\Delta}
$$

where $\Delta=M^{2}-x(1-x) k^{2}$

As expected, the integral depends on the regularization scheme.

For the case of a non-renormalizable theory (the contact potential), the specification of regularization scheme becomes part of the model. One can simply treat $\Lambda$ as a parameter of the model.

For the linear case, we consider the renormalized integral:

$$
\Pi\left[k_{0}, \vec{k}\right]=\left[\frac{I_{k_{0}, \vec{k}}}{\vec{k}^{2}}-\frac{I_{k_{s 0}, \vec{k}_{s}}}{{\overrightarrow{k_{s}}}^{2}}\right]\left(\vec{k}^{2}\right)
$$

where $k_{s 0}, \overrightarrow{k_{s}}$ is the subtraction point, note that the renormalized integral is scheme independent.

Now we focus on the computation of the ring integral at finite temperature and density. The relevant Matsubara sum is given by: 


$$
\Pi=\frac{i}{\beta} \sum_{\omega_{n}^{\prime}} \int \frac{d^{3} k^{\prime}}{(2 \pi)^{3}} \operatorname{Tr}\left[\gamma^{0} S_{k^{\prime}} \gamma^{0} S_{k^{\prime}-k}\right]
$$

It is useful to separate the sum into the vacuum part and the matter part:

$$
\Pi=\Pi_{\mathrm{vac}}+\Pi_{\mathrm{mat}}
$$

with $\Pi_{\mathrm{vac}}$ as given above.

For the matter piece, we again present the result in the perturbative limit:

$$
\begin{aligned}
\Pi_{\text {mat }}= & -\frac{i}{\pi^{2}} \int d k^{\prime} \frac{k^{\prime 2}}{\epsilon^{\prime}}\left(n_{\epsilon^{\prime}}+\bar{n}_{\epsilon^{\prime}}\right) \\
& {\left[1+\frac{1}{8 k^{\prime}|\vec{k}|}\left[\left(4 \epsilon^{\prime} k_{0}-4 \epsilon^{\prime 2}-k^{2}\right)\right] \ln \frac{k^{2}-2 k_{0} \epsilon^{\prime}+2 k^{\prime}|\vec{k}|}{k^{2}-2 k_{0} \epsilon^{\prime}-2 k^{\prime}|\vec{k}|}+\right.} \\
& \left.\frac{1}{8 k^{\prime}|\vec{k}|}\left[\left(-4 \epsilon^{\prime} k_{0}-4 \epsilon^{\prime 2}-k^{2}\right)\right] \ln \frac{k^{2}+2 k_{0} \epsilon^{\prime}+2 k^{\prime}|\vec{k}|}{k^{2}+2 k_{0} \epsilon^{\prime}-2 k^{\prime}|\vec{k}|}\right]
\end{aligned}
$$

where

$$
\begin{aligned}
n(E) & =\frac{1}{1+e^{\beta(E-\mu)}} \\
\bar{n}(E) & =\frac{1}{1+e^{\beta(E+\mu)}} .
\end{aligned}
$$

Several interesting limits of the above include:

- $k^{0}=0$, general $|\vec{k}|$

$$
\frac{-i}{\pi^{2}} \int d k^{\prime} \frac{k^{\prime 2}}{\epsilon^{\prime}}[n+\bar{n}]\left[1+\frac{1}{8 k^{\prime}|\vec{k}|}\left(-4 \epsilon^{\prime 2}+|\vec{k}|^{2}\right) \ln \left(\frac{|\vec{k}|-2 k^{\prime}}{|\vec{k}|+2 k^{\prime}}\right)^{2}\right]
$$


- $k^{0}=0,|\vec{k}| \rightarrow 0$

$$
\frac{-i}{\pi^{2}} \int d k^{\prime} \frac{k^{\prime 2}}{\epsilon^{\prime}}(n+\bar{n})\left(1+\frac{\epsilon^{\prime 2}}{k^{\prime 2}}\right)
$$

Especially, at $M \rightarrow 0$, we obtain the well known result

$$
\lim _{|\vec{k}| \rightarrow 0} I_{\text {mat }}\left[k_{0}=0,|\vec{k}|, M \rightarrow 0\right]=-i\left(\frac{1}{3} T^{2}+\frac{\mu^{2}}{\pi^{2}}\right)
$$

- $k^{0} \neq 0,|\vec{k}|=0$

For this case, the integral becomes

$$
\Pi_{\text {mat }} \rightarrow \frac{i}{\pi^{2}} \int d k^{\prime}(n+\bar{n}) \frac{k^{\prime 2}}{\epsilon_{1}}\left[\frac{\epsilon_{1}\left(2 \epsilon_{1}-k_{0}\right)}{k_{0}\left(k_{0}-2 \epsilon_{1}\right)}+k_{0} \rightarrow-k_{0}\right],
$$

which is clearly zero.

As a simple illustration of how the ring can regulate the infrared divergence of the linear potential, we consider only the matter part of the ring in the static, long wavelength and massless limit (as shown in equation 3.32). The ring potential becomes

$$
V_{\text {ring }}=\frac{V}{1+V \frac{1}{2} i \Pi} \longrightarrow \frac{6 \pi b}{k^{4}+(3 \pi b) n_{f}\left(\frac{1}{3} T^{2}+\frac{\mu^{2}}{\pi^{2}}\right)} .
$$

Clearly, the potential is no longer infrared divergent after the introduction of the ring integral. 


\subsection{PHASE STRUCTURE OF QCD}

QCD at finite temperature and density is a very active field of research [30]. The knowledge of the equation of state of $\mathrm{QCD}$, especially, the nature of chiral phase transition, is essential for understanding experimental data. Mapping out the phase diagram of QCD is a gigantic task. It demands a complete understanding of both the non-perturbative and perturbative aspects of the strong interactions. In the meantime, competing theories, usually applicable only in a small region of the phase diagram, are making various speculations on the properties of QCD phase structures. The final settlement of issues, however, should await the discovery in the experiments at RHIC, LHC and FAIR. [31, 32, 33].

The current consistent picture from lattice calculations seems to suggest that at $\mu=0$, the phase transition is second order in nature for massless quarks. The agreed value of $T_{c}$ is $170 \mathrm{MeV}[34]$.

At $T=0$, while lattice study suffers from the notorious negative sign problem, many

models $[18,35]$ suggest that the chiral symmetry restoration at finite $\mu$ is first order in nature. If this is true, it may be argued that a tricritical point exists in QCD: a change of order of phase transition from second order to first order [36].

Here is an illustration for a possible phase diagram of QCD: 


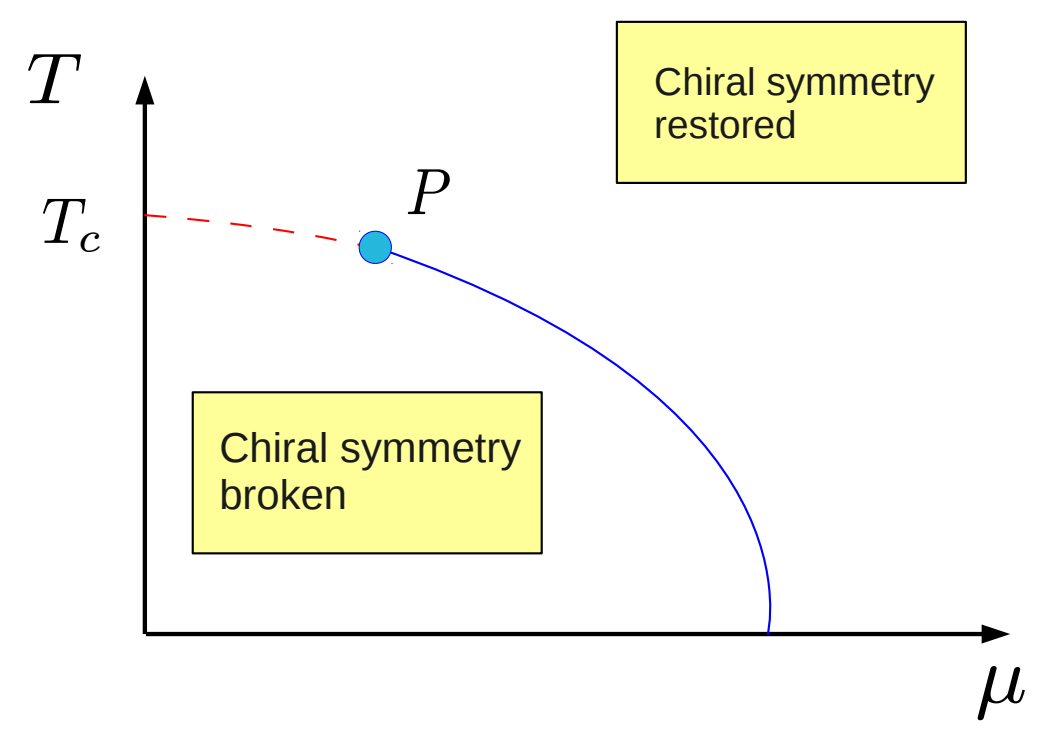

Figure 7: The schematic phase diagram of QCD with 2 massless quarks. The dashed line (red) represents second order transition, the solid line (blue) is the first order transition. The point $\mathrm{P}$ is tricritical. $T_{c} \approx 0.17 \mathrm{GeV}$ from lattice calculations.

Note that in real QCD with non-zero current quark masses, the second order phase transition becomes a crossover. The tricritical point would then be a critical end-point for the line of first order phase transition.

\subsection{PHASE STRUCTURE OF POTENTIAL MODELS}

In the following, we will study the phase structures for various potential models. We will employ the zero momentum limit of the constituent mass function $M_{0}=M[k \rightarrow 0 ; T, \mu]$ as the proxy for the chiral condensate. By plotting $M_{0}$ as a function of temperature and chemical potential, we can determine the phase structures of the contact and potential 
models. We are especially interested in how the inclusion of polarization effects alters the phase diagrams.

The coupled gap equations are solved with various approaches. Typically, we discretize the equations on the momentum grids and employ minimization routine based on modified Levenburg-Marquardt algorithm. We also attempt iterative methods and sometimes combinations of both iteration and minimization. A variety of analytic approximations are applied to the calculation of $\Pi[k]$ as the full momentum dependence of the ring is usually the most time-consuming part of the calculation. The results presented below are borne out of several months of computer works.

\subsubsection{RESULTS: CONTACT POTENTIAL AT $T>0$}

Recall that for the contact potential, one requires an UV cutoff $\Lambda$ to regulate the integrals. Also there is a critical coupling $\lambda_{c}=6 \pi^{2}$, below which chiral symmetry breaking ceases to exist $^{8}$.

For the purpose of discussion, we set $\lambda=1.5 \lambda_{c}$ and fix the scale at $\Lambda=370 \mathrm{MeV}$. The dynamical quark mass, chiral restoration temperature and the critical chemical potential are: $M[T=0, \mu=0] \approx 260 \mathrm{MeV}, T_{c} \approx 150 \mathrm{MeV}$ and $\mu_{c} \approx 300 \mathrm{MeV}$. The condensate works out to be $(-150 \mathrm{MeV})^{3}$, a factor of 2 too small compared to the physical value. The numerical values of these observables shift slightly with other choices of the parameters.

Now we turn to the investigation of phase structure.

3.4.1.1 PHASE MAP Firstly, we present the phase map ${ }^{9}$ for the case of bare contact potential:

\footnotetext{
${ }^{8}$ Again we shall express all quantities in $\Lambda$.

${ }^{9}$ The term "phase map" refers to the three dimensional plot of the order parameter against temperature and chemical potential. We reserve the term "phase diagram" to describe the plane of phase transitions: the graph of transition temperature versus chemical potential.
} 


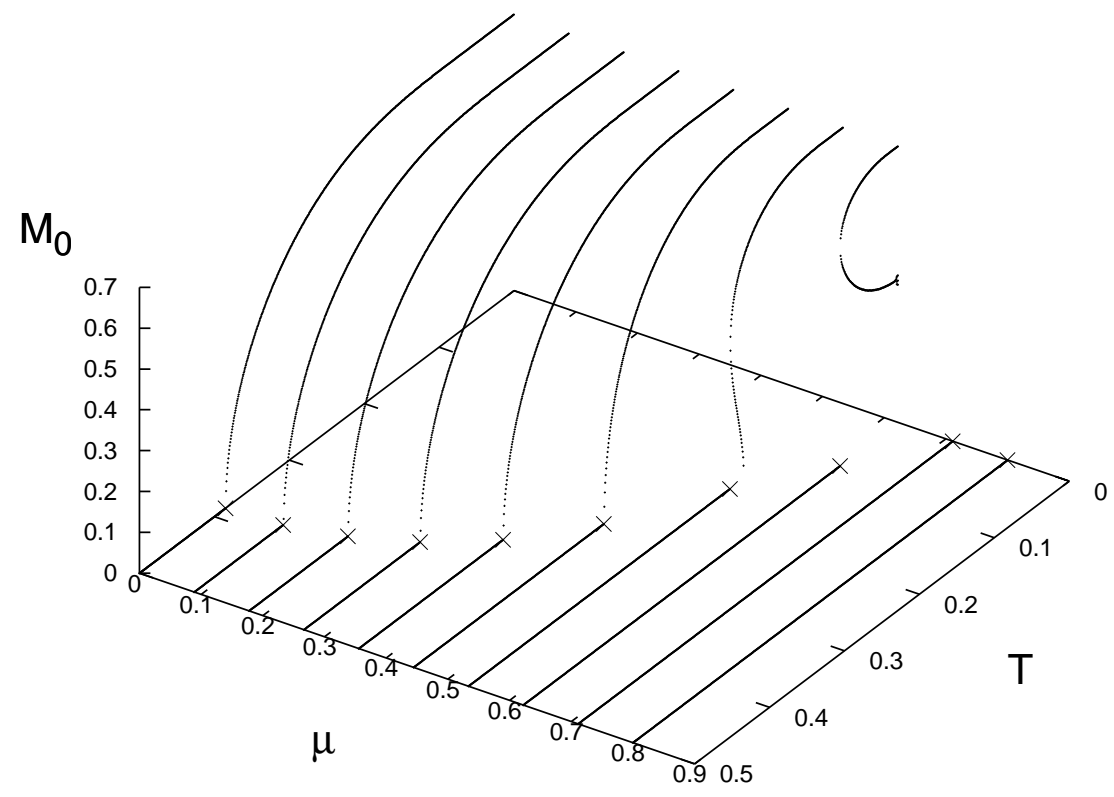

Figure 8: Dynamical mass versus temperature and density for bare contact model. $\lambda=3 \lambda_{c}$

We make the following important observations:

- The $M_{0}=0$ plane of the phase map defines the phase diagram. At $\mu=0$, we observe $T_{c}=0.38$.

- The chiral symmetry phase transition is second order for all chemical potential below $\mu^{\star}\left(\lambda=1.5 \lambda_{c}\right) \approx 0.53$. Afterward, the transition is first order.

- Multiple solutions (lower band) start to emerge for larger $\mu$ accompanying the first order transition, they are expected to have higher free energy as demonstrated by their lack of continuity with the well-behaved low- $\mu$ solutions. 


\subsubsection{ORDER OF PHASE TRANSITION AND THE EXISTENCE OF MUL-} TIPLE SOLUTIONS For the case of bare contact potential, it becomes particularly transparent to understand the order of phase transitions.

The solution to the gap equation in contact case is a constant function in momentum, this allows easy extraction of solution using root-finding method.

Recall that the gap equation takes the form:

$$
\begin{aligned}
1 & =\frac{1}{3 \pi^{2}} \lambda \int^{\Lambda} d k^{\prime} k^{\prime 2} \frac{1}{E^{\prime}}[1-n-\bar{n}] \\
\mu^{\prime} & =\mu+\frac{1}{3 \pi^{2}} \lambda \int^{\Lambda} d k^{\prime}[n-\bar{n}] .
\end{aligned}
$$

Define

$$
\mathcal{F}[T, \mu, M]=\int^{\Lambda} d k^{\prime} k^{\prime 2} \frac{1}{E^{\prime}}[1-n-\bar{n}]
$$

given $T$ and $\mu$, the equation

$$
\mathcal{F}^{T \mu}[M]=1
$$

defines the solution of the gap equation.

The study of $\mathcal{F}$ allows us to understand the order of the phase transition and the origin of the multiple solutions in region of larger $\mu$. As we shall see, depending on the value of $\mu$, the $\mathcal{F}$ function can behave very differently. We shall focus on the structure of $\mathcal{F}$ function in both the small $\mu$ and large $\mu$ regions.

A typical $\mathcal{F}$ function for small $\mu$ looks like: 


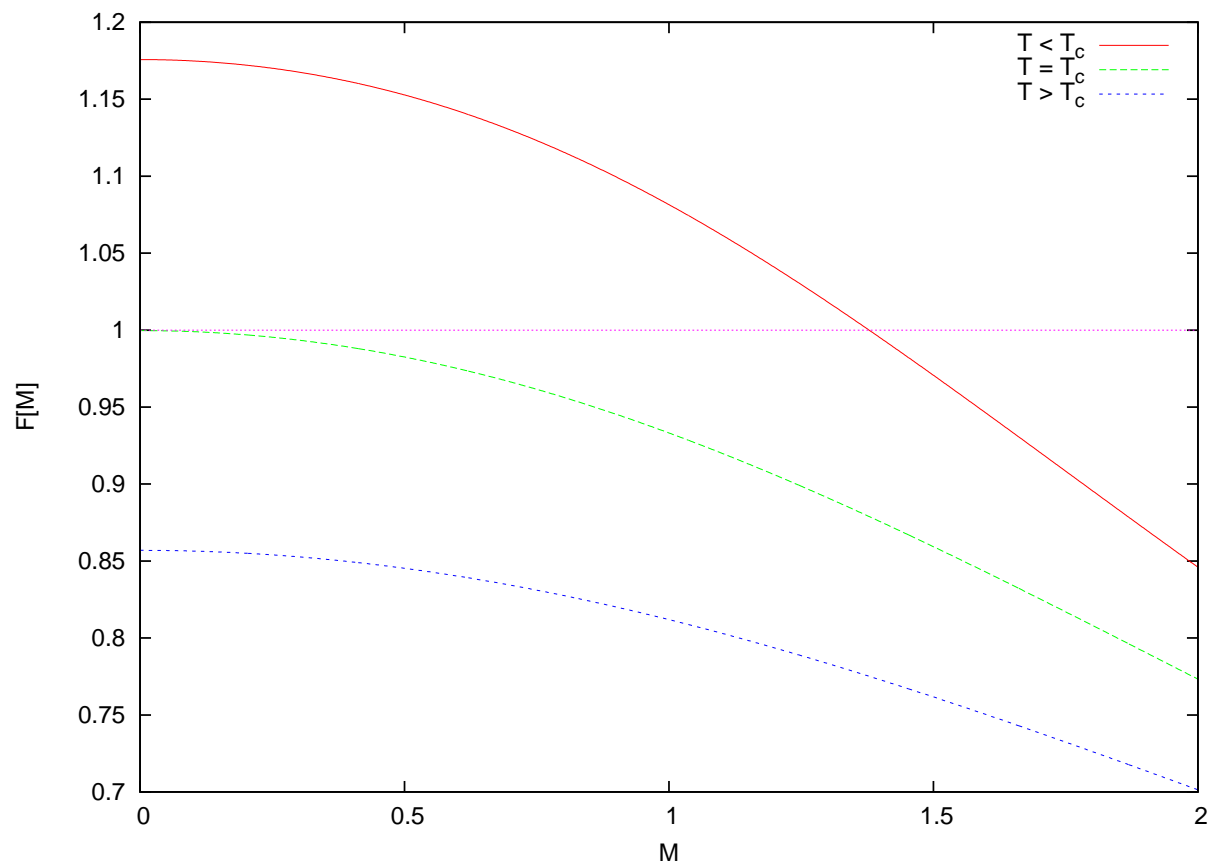

Figure 9: Typical $\mathcal{F}[M]$ function for small $\mu$. The graph shows that the phase transition is second order in nature.

Clearly, we see that only one solution exists for each $T, \mu$. Also we see that the mass goes to zero when $T \rightarrow T_{c}$, signaling a second order phase transition.

Similarly, we look at a typical $\mathcal{F}$ function for large $\mu$ : 


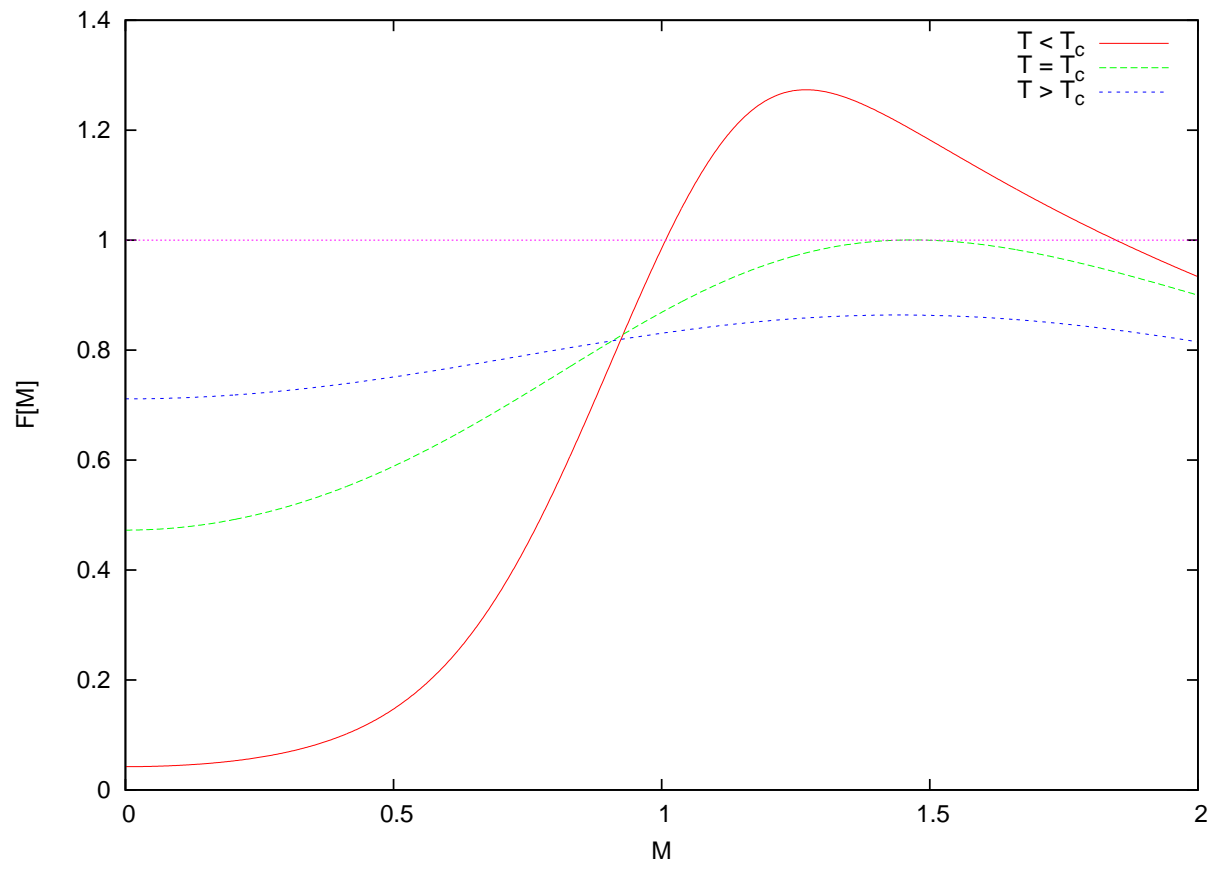

Figure 10: Typical $\mathcal{F}[M]$ function for large $\mu$. The diagram shows that the phase transition is first order in nature

We observe that two solutions exist. Also we see that the mass does not go to zero when $T \rightarrow T_{c}$, signifying a first order phase transition.

\subsubsection{PHASE MAPPING BY DIFFERENTIAL EQUATION For reference} purpose, we present a completely different method to map out the phase diagram. We illustrate this method for the contact case:

The gap equation reads:

$$
1=\frac{1}{3 \pi^{2}} \lambda \int^{\Lambda} d k^{\prime} k^{\prime 2} \frac{1}{\sqrt{k^{\prime 2}+M^{2}}}[1-n-\bar{n}]
$$


with

$$
\begin{aligned}
n & =\frac{1}{1+e^{\beta\left(\epsilon-\mu^{\prime}\right)}} \\
\bar{n} & =\frac{1}{1+e^{\beta\left(\epsilon+\mu^{\prime}\right)}} \\
\epsilon & =\sqrt{{k^{\prime}}^{2}+M^{2}} .
\end{aligned}
$$

Differentiating with respect to $\beta$ on both side, we get

$$
0=\int^{\Lambda} d k^{\prime} k^{\prime 2} \frac{d}{d \beta}\left[\frac{1}{\epsilon}(1-n-\bar{n})\right]
$$

and with some algebra, we reach

$$
\begin{aligned}
\frac{d M}{d \beta} & =\frac{\int_{0}^{1} d k^{\prime} k^{\prime 2} \frac{1}{\epsilon}\left[n(1-n)\left(\epsilon-\mu^{\prime}\right)+\bar{n}(1-\bar{n})\left(\epsilon+\mu^{\prime}\right)\right]}{\int_{0}^{1} k^{\prime 2} \frac{1}{\epsilon^{2}}\left[(1-n-\bar{n}) \frac{M}{\epsilon}-[n(1-n)+\bar{n}(1-\bar{n})] \beta M\right]} \\
\frac{d M}{d \mu^{\prime}} & =\frac{\int_{0}^{1} d k^{\prime} k^{\prime 2} \frac{1}{\epsilon} \beta[-n(1-n)+\bar{n}(1-\bar{n})]}{M \int_{0}^{1} k^{\prime 2} \frac{1}{\epsilon^{2}}\left[(1-n-\bar{n}) \frac{1}{\epsilon}-\beta[n(1-n)+\bar{n}(1-\bar{n})]\right]} .
\end{aligned}
$$

Given the initial conditions (the non-trivial solution of the gap equation at zero temperature and zero density), we can evolve the equations and map out the phase diagram. This approach is tested and gives identical solutions as presented above. 
3.4.1.4 THE EFFECT OF $\lambda$ It is also interesting to see how the phase diagram depends on the coupling $\lambda$. We define $\xi=\frac{\lambda}{6 \pi^{2}}$ and study the collection of phase diagrams:

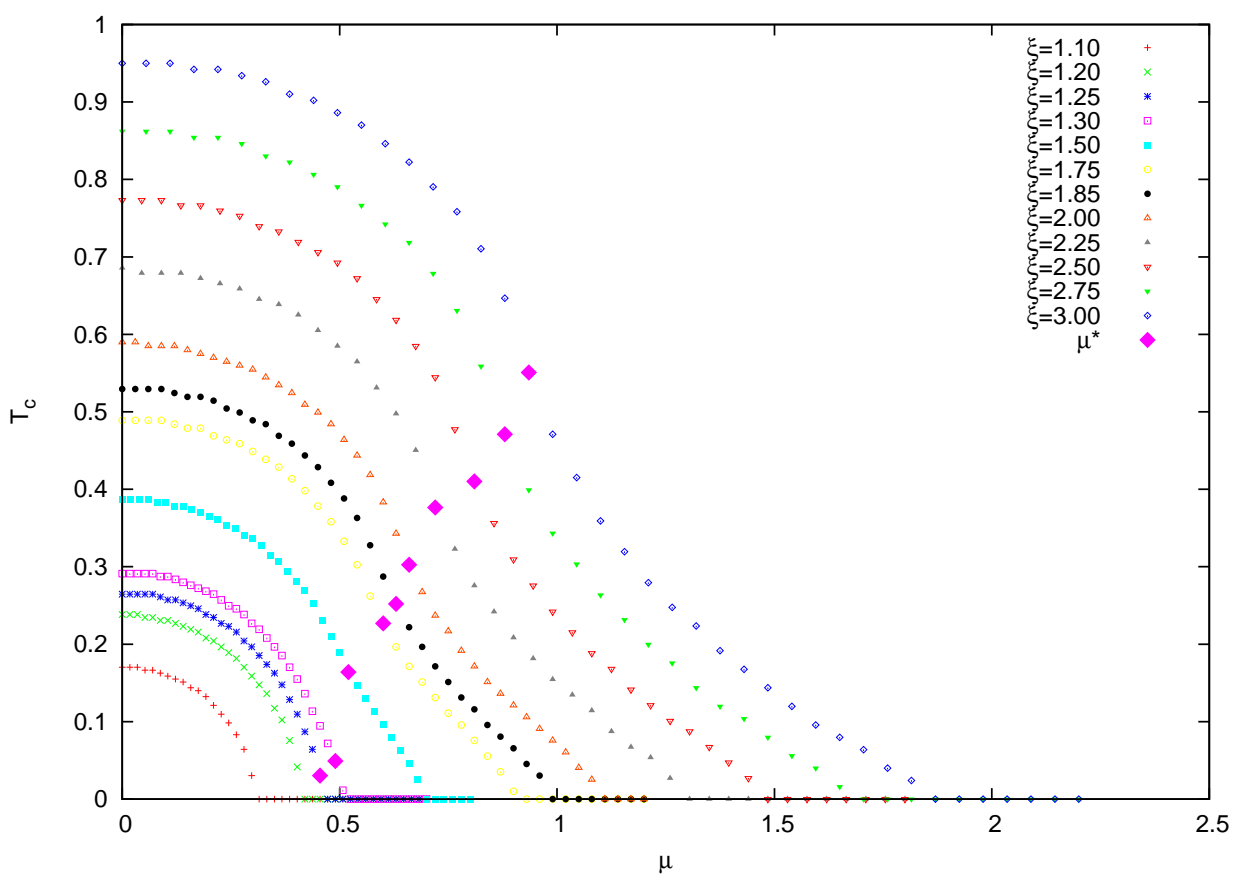

Figure 11: The phase diagram for bare contact interaction at various couplings. $\mu^{\star}$ signifies the point at which the order of phase transition goes from second order to first order.

First order phase transitions become more and more prominent as $\lambda$ increases (the points to the right of $\mu^{\star}$ ). Also, we observe an approximate linear scaling relations: (below $\xi=1.2$, first order phase transitions cease to exist, the phase transitions are all second order in nature)

$$
\mu_{c}[\lambda] \approx 2 T_{c}[\lambda] \approx 2 \mu^{\star}[\lambda]
$$


3.4.1.5 EFFECTS OF RING Lastly we investigate the effect of ring inclusion by plotting the phase map for the following three potential:

- $V_{1}=\lambda$

- $V_{2}=\frac{\lambda}{1+i \frac{1}{2} \Pi[0,0] \lambda}$

- $V_{3}=\frac{\lambda}{1+i \frac{1}{2} \Pi[0, \vec{k}] \lambda}$

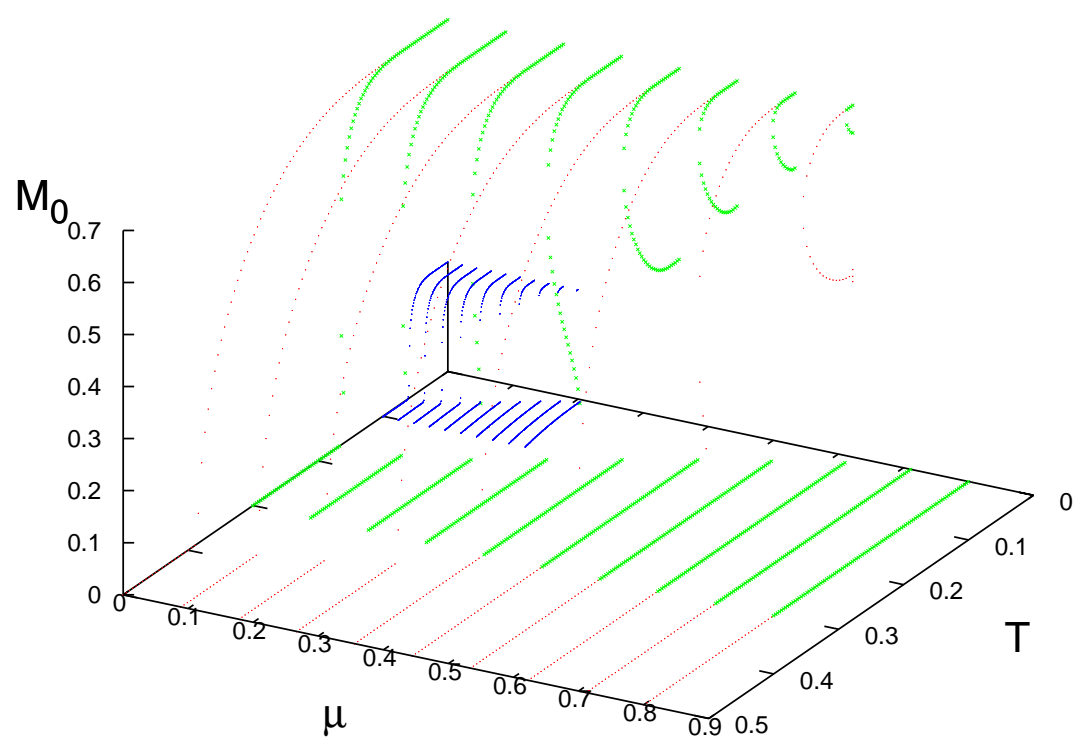

Figure 12: Dynamical mass versus temperature and density for the contact model with: (i) bare potential (red) (ii) static, zero momentum ring (green) (iii) static, momentum dependent ring (blue). For all cases, $\lambda=1.5 \lambda_{c}$

We observe that the critical temperature is strongly affected, in fact, it drops from 0.38 to 0.17 with the inclusion of static, zero momentum ring $\Pi[0,0]$, even further to 0.06 with the static, full momentum ring. Moreover, first order phase transitions become dominant once we include the polarization effects. 


\subsubsection{RESULTS: LINEAR POTENTIAL AT $T>0$}

For the linear potential, one faces the problem of infrared divergence. We shall illustrate both the Alkofer's prescription (AAL) and the ring approximation in the discussion. Within AAL, we find: $T_{c} \approx 38 \mathrm{MeV}, \mu_{c} \approx 75 \mathrm{MeV}, M_{0} \approx 80 \mathrm{MeV}$ and the condensate $(-110 \mathrm{MeV})^{3}$. All these fall below the expected values. However, employing a string tension $b \approx 1.8 \mathrm{GeV}$ bring them all into reasonable agreement with the physical values. Unfortunately, such a large value of string tension is in severe conflict with quark model phenomenology and lattice data.

3.4.2.1 PHASE MAP AND PHASE DIAGRAM We will first study the phase map in Alkofer's prescription. 


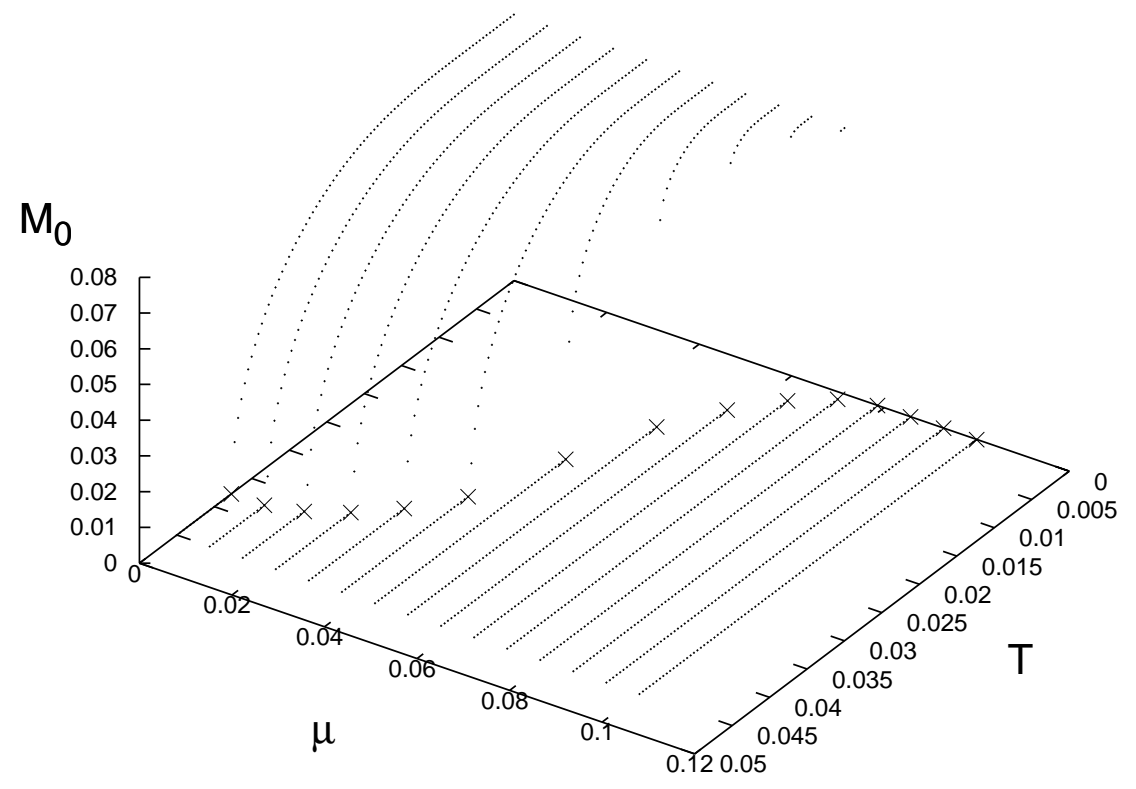

Figure 13: Dynamical mass versus temperature and density for bare linear model (AAL). All quantities in $\mathrm{GeV}$. 


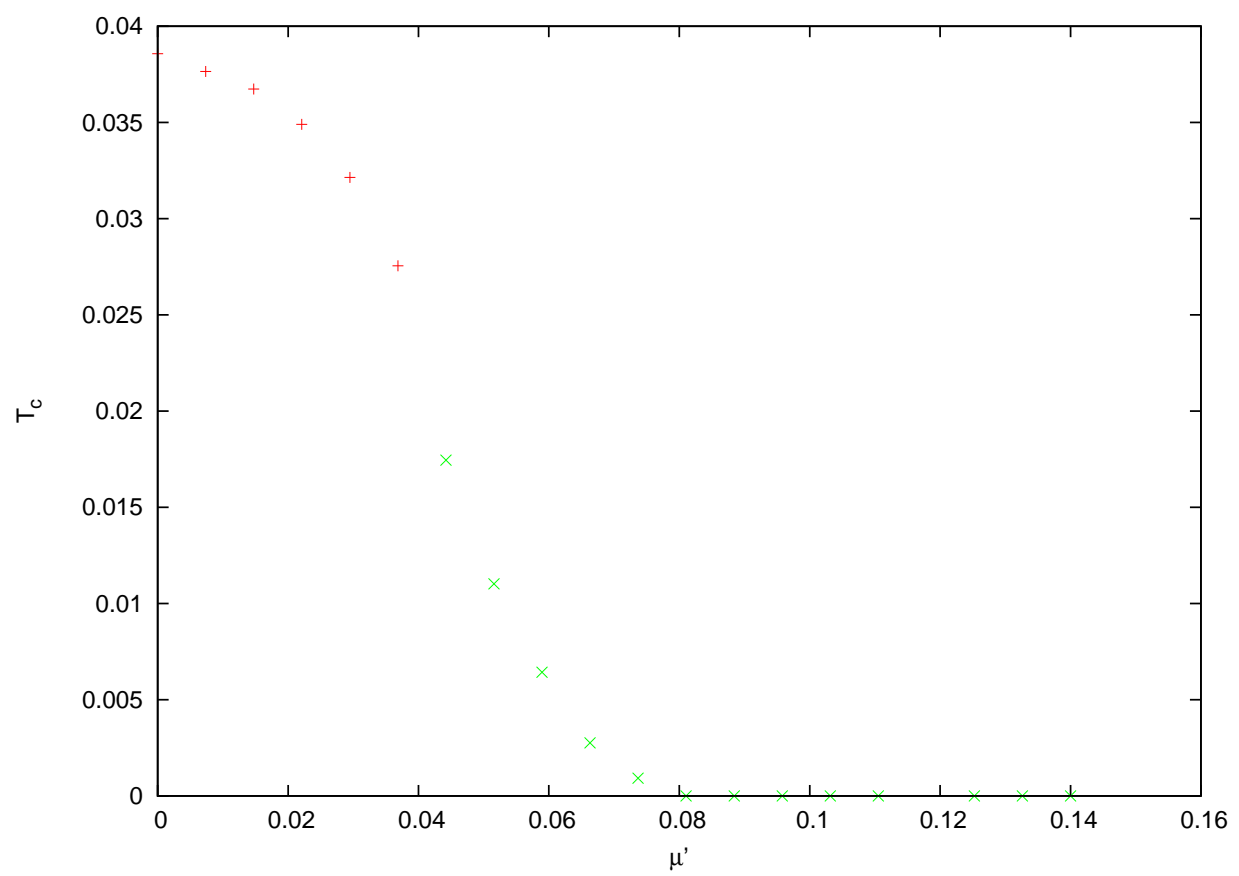

Figure 14: Phase diagram for bare linear model (AAL). The red and green dots correspond to second and first order phase transition respectively. All quantities in GeV.

The phase map and phase diagram for the linear case display similar features to those of the contact potential. Again, we observe a change of the order of phase transition from second order to first order at $\mu^{\star}=43 \mathrm{MeV}$. The existence of tricritical point is consistent with the expectation for real QCD.

3.4.2.2 EFFECTS OF RING Next we investigate the ring approximation approach to the infrared divergence problem. It is important to note that the vacuum part of the ring $\Pi_{\text {vac }}$ is non-zero and can drastically alter the long range behavior of the potential. This will in turn ruin the agreement with heavy quark spectroscopy. Hence, we shall treat $\Pi_{\text {vac }}$ as if it has already been incorporated in the $\frac{1}{k^{4}}$ potential, and only consider the matter piece of 
the ring.

The polarization effect introduces explicit temperature and density dependence to the

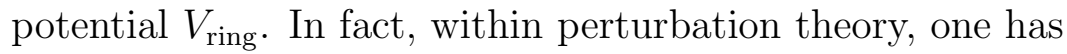

$$
V_{\text {ring }}=\frac{6 \pi b}{k^{4}+(3 \pi b) n_{f}\left(\frac{1}{3} T^{2}+\frac{\mu^{2}}{\pi^{2}}\right)} .
$$

The resulting potential is linear in small distance, while approaching zero at large distance, i.e the potential is no longer confining at finite temperature.

Shown below is the phase map for static, long wavelength ring approximation $\Pi_{\text {mat }}\left[k_{0}=\right.$ $0, \vec{k} \rightarrow 0]:$

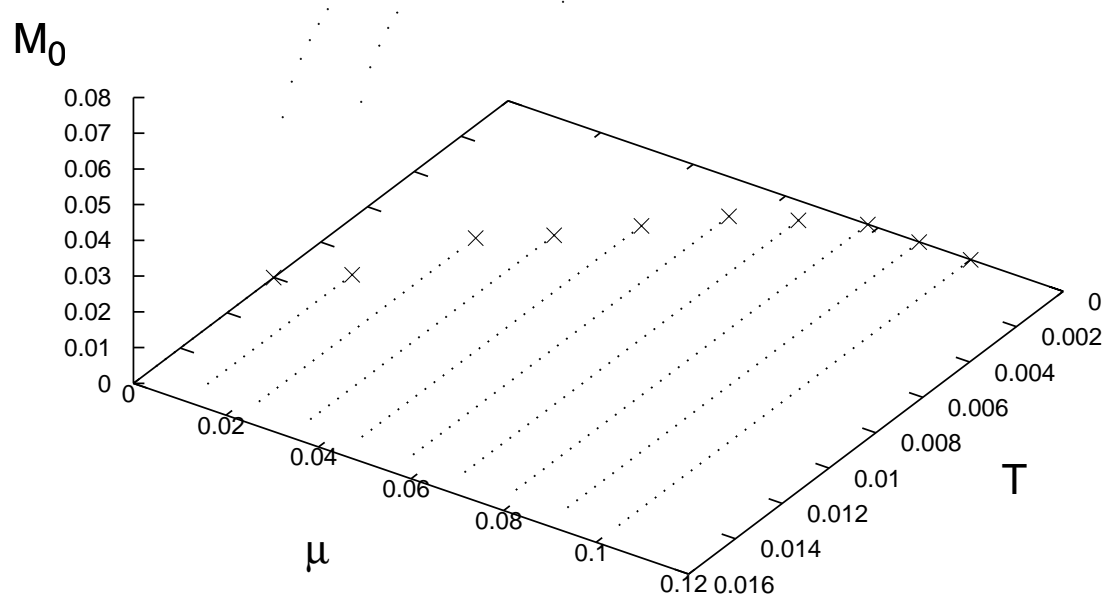

Figure 15: Dynamical mass versus temperature and density for linear potential with matter part of ring function under static, long wavelength limit. All quantities in GeV. 
We observe that the critical temperature drops from $38 \mathrm{MeV}$ in $\mathrm{AAL}$ to $10 \mathrm{MeV}$ in $\Pi_{\text {mat }}[0,0]$ approximation while $\mu_{c}$ remains unchanged. Parallel to the contact case, first order phase transitions become dominant as we include the ring, and the tricritical point ceases to exist.

\subsection{CONCLUSIONS}

We have investigated two types of confinement models motivated by Coulomb gauge QCD: contact and linear potentials.

For the contact potential, we present the phase map for both the bare and ring versions. The bare contact model exhibits many interesting features such as the existence of tricritical point and the approximate linear scaling of $T_{c}, \mu_{c}$ with the coupling. The numerical values of the constituent mass, condensate, critical temperature and chemical potential can be brought into rough agreement with the physical expectations in QCD by an appropriate choice of parameters. However, the incorporation of polarization effects drastically affects the phase structure. The most prominent effects are the substantial drop in the critical temperature (ruining phenomenology) and the dominance of first order phase transition.

For the linear potential, both the AAL prescription for the bare potential and the ring approximation yield a chiral phase transition, contrary to the expectation of Davis and Matheson. Especially, given the fact that large $N_{c}$ limit tends to suppresses quark loop effects, one can argue the bare linear potential studied here is an implementation of the large $N_{c}$ scenario. Our results then support the idea of quarkyonic matter argued by Pisarski and McLerran [37]: the existence of confining but chirally symmetric phase. The summation of ring diagrams is motivated as the resolution of the infrared divergence problem in the linear potential. The numerical values for the constituent mass, condensate, critical temperature and density can attain reasonable QCD limit if the string tension $b$ is increased to $1.8 \mathrm{GeV}$. Unfortunately this is at odds with the well-established quark model phenomenology and lattice data, which requires $b=0.2 \mathrm{GeV}$. Therefore, it appears that naive static potential model fail to describe the thermal properties of QCD. 
One may speculate such failure is due to the negligence of the transverse gluon in the model. However, the study by Kloker [38] suggested that in the context of Coulomb gauge, no considerable increase in mass function can be found even when the transverse gluon effects are incorporated. Also, improving the truncation by including ring diagrams leads to a worse agreement with data. We therefore judge that the problem lies in the model itself: one cannot fulfill the many-body aspects of the theory (obtaining the right dynamical mass) without compromising its few-body aspects (significantly alter the string tension $b$, ruining meson spectrum phenomenology). 


\subsection{QED3 AT $T=0$}

In this chapter ${ }^{1}$, we will study dynamical chiral and parity symmetry breaking of threedimensional quantum electrodynamics (QED3). The theory is popular for many reasons. First, the confining property motivates its use as a model of QCD at high temperature in the large $N_{f}$ limit, where the non-abelian nature of the theory is suppressed [40]. In addition, the capability of large scale dynamical masses generation makes it particularly relevant in the modeling of beyond Standard Model physics [41]. More recently, QED3 has also been used as a model field theory for condensed matter systems such as high $T_{c}$ superconductors and graphene.

One interesting feature of QED3 is that it is possible to introduce a Chern-Simons-like term in the Lagrangian, which corresponds to a topological photon mass, and hence will break parity and time reversal symmetries. Within the two-component fermions formalism, it is possible to generate such a term dynamically at one loop order. Hence, the theory exhibits both chiral and parity symmetry breaking.

The term "chiral symmetry" requires some explanations. Strictly speaking, as we shall see in later section, in the context of 2-spinor representation, there is nothing to generate a chiral symmetry that would be broken by a fermion mass term $m \bar{\psi} \psi$. That is, the massless theory has no more symmetry than the massive one. However, if we consider $N_{f}$ fermions in the usual 4-spinor formalism, the theory exhibits a global $U\left(2 N_{f}\right)$ symmetry [41] that can be broken by a fermion mass term into $U\left(N_{f}\right) \times U\left(N_{f}\right)$. It is in this context that we use the term "chiral symmetry" in the present discussion.

In the study, we shall employ a variety of truncation schemes for the Schwinger-Dyson equations. Particularly interesting is the gauge symmetry preserving Ball-Chu vertex. The

\footnotetext{
${ }^{1}$ The research in this chapter is published in "Parity symmetry in QED3" [39].
} 
robustness of a truncation can be examined by a theorem due to Coleman and Hill [42], on which we shall discuss further in the later section. Results for both parity and chiral symmetry breaking will be presented, with comments and discussions follow towards the end of the chapter.

\subsection{LAGRANGIAN}

QED3 is quantum electrodynamics in $(2+1)$ spacetime. The Lagrangian is given by

$$
L_{Q E D}=\int d^{3} x \bar{\psi}(i \not \partial-m-g A) \psi-\frac{1}{4} F^{2}-\frac{1}{2 \xi}(\partial A)^{2}
$$

where $F_{\mu \nu}=\partial_{\mu} A_{\nu}-\partial_{\nu} A_{\mu}$.

One key feature of the theory is that in three dimensional spacetime, $\psi$ has units of energy $E$, the photon field $A$ has units of $\sqrt{E}$ and most importantly,

$$
\alpha=\frac{g^{2}}{4 \pi} \sim E
$$

This fact corresponds to the theory being super-renormalizable. It also provides a natural energy scale, through which we shall express all our physical quantities.

\section{2-SPINOR FORMALISM}

In QED3, the Dirac algebra can be realized by the three $2 \times 2$ Pauli matrices [43].

Recall the Pauli matrices $\sigma_{1}, \sigma_{2}$ and $\sigma_{3}$ satisfy

$$
\begin{aligned}
{\left[\sigma_{i}, \sigma_{j}\right] } & =2 i \epsilon_{i j k} \sigma_{k} \\
\left\{\sigma_{i}, \sigma_{j}\right\} & =2 \delta_{i j}
\end{aligned}
$$


With the metric

$$
g^{\mu \nu}=\left(\begin{array}{ccc}
1 & 0 & 0 \\
0 & -1 & 0 \\
0 & 0 & -1
\end{array}\right)
$$

the Dirac algebra is realized once we identify:

$$
\gamma^{0} \rightarrow \sigma^{3}
$$

$$
\gamma^{1}, \gamma^{2} \rightarrow i \sigma^{1}, i \sigma^{2}
$$

It is easy to check that:

$$
\left\{\gamma^{\mu}, \gamma^{\nu}\right\}=2 g^{\mu \nu}
$$

Operationally, in a quantum field theory, the main difference between a 2-spinor formalism and the usual 4-spinor formalism is in the evaluation of traces. Through some Dirac gymnastics, we obtain:

$$
\begin{aligned}
\left\{\gamma^{\mu}, \gamma^{\nu}\right\} & =2 g^{\mu \nu} \\
{\left[\gamma^{\mu}, \gamma^{\nu}\right] } & =-2 i \epsilon^{\mu \nu \rho} \gamma_{\rho} \\
\gamma^{\mu} \gamma^{\nu} & =g^{\mu \nu}-i \epsilon^{\mu \nu \rho} \gamma_{\rho} .
\end{aligned}
$$

From these, we can obtain the following useful identities: 


$$
\begin{aligned}
\operatorname{Tr}\left[\gamma^{\mu}\right] & =0 . \\
\operatorname{Tr}\left[\gamma^{\mu} \gamma^{\nu}\right] & =2 g^{\mu \nu} \\
\operatorname{Tr}\left[\gamma^{\mu} \gamma^{\nu} \gamma^{\rho}\right] & =-2 i \epsilon^{\mu \nu \rho} \\
\operatorname{Tr}\left[\gamma^{\mu} \gamma^{\nu} \gamma^{\alpha} \gamma^{\beta}\right] & =2\left\{g^{\mu \nu} g^{\alpha \beta}-g^{\mu \alpha} g^{\nu \beta}+g^{\mu \beta} g^{\nu \alpha}\right\}
\end{aligned}
$$

Note that within the 2-spinor formalism, the trace of 3 gamma's (and other odd \#'s above

3 ) is not zero! This property directly leads to the existence of dynamical parity symmetry breaking, a point which we shall discuss later.

\subsection{SCHWINGER-DYSON EQUATIONS}

\subsubsection{WARD IDENTITY AND TRUNCATION}

We will be working on the Schwinger Dyson equations of QED3 with the 2-spinor formalism. To do that, we need a truncation scheme, we choose to perform it by employing a vertex that satisfies the Ward identity: the Ball-Chu vertex, so that we can perform a non-perturbative study of the gap equations in a gauge invariant setting.

The Ball-Chu vertex, expressed in terms of the fermion propagator functions, can be written as:

$$
\Gamma_{B . C}^{\nu}[p, q]=\frac{A_{q}+A_{p}}{2} \gamma^{\nu}+\frac{(q+p)^{\nu}}{q^{2}-p^{2}}\left[\left(A_{q}-A_{p}\right) \frac{(q+p)_{\rho} \gamma^{\rho}}{2}-\left(B_{q}-B_{p}\right)\right]
$$

where $p, q$ being the momenta of the incoming and outgoing fermion respectively.

One can verify easily that the Ball-Chu vertex satisfies the Ward identity:

$$
(q-p)_{\nu} \Gamma_{B . C}^{\nu}[p, q]=S_{q}^{-1}-S_{p}^{-1}
$$


where

$$
S_{p}^{-1}=A_{p} \not p-B_{p}
$$

It is useful to define

$$
\begin{gathered}
\Omega_{1}=\frac{A_{q}+A_{p}}{2} \\
\Omega_{2}=\frac{1}{q^{2}-p^{2}} \frac{A_{q}-A_{p}}{2} \\
\Omega_{3}=-\frac{1}{q^{2}-p^{2}}\left(B_{q}-B_{p}\right) \\
R^{\nu}=(q+p)^{\nu}
\end{gathered}
$$

such that the Ball-Chu vertex reads:

$$
\Gamma_{B . C}^{\nu}[p, q]=\Omega_{1} \gamma^{\nu}+\Omega_{2} R^{\nu} R_{\rho} \gamma^{\rho}+\Omega_{3} R^{\nu}
$$

The familiar rainbow ladder truncation is obtained by approximating the full vertex with the bare one. It can be described in a unified manner in the current formalism by setting:

$$
\begin{array}{r}
\Omega_{1}=1 \\
\Omega_{2}=\Omega_{3}=0
\end{array}
$$

It should be made clear that the Ward identity only restrict the longitudinal part of the fermion-photon interaction. The transverse piece, on the other hand, should be formally determined by the Schwinger Dyson equations, or subjected to further modeling. For our study (with the exception of Curtis-Pennington vertex), as an implicit assumption of the truncation scheme, we shall neglect the transverse contribution of the vertex. 


\subsubsection{GAP EQUATION FOR THE FERMIONS}

We shall consider the following equation:
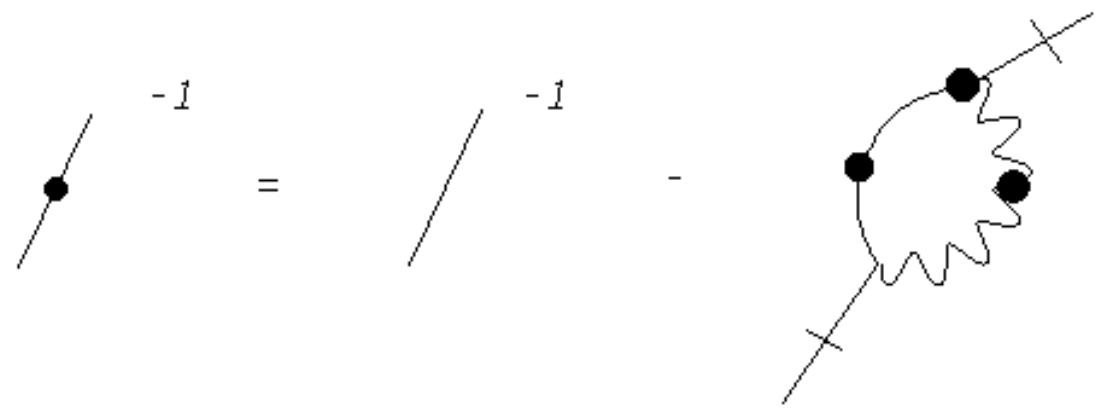

Figure 16: Schwinger-Dyson equations for fermions.

which translates into

$$
\begin{aligned}
-i S^{-1} & =-i S_{0}^{-1}-\left[\left(-i g \Gamma_{B . C}^{\mu}\right) i S\left(-i g \gamma^{\nu}\right) i D_{\mu \nu}\right] \\
S^{-1} & =S_{0}^{-1}-i g^{2}\left[\Gamma_{B . C}^{\mu} S \gamma^{\nu} D_{\mu \nu}\right] .
\end{aligned}
$$

With the Ansätz:

$$
S_{p}=\frac{1}{A_{p} \not p-B_{p}}
$$

we have

$$
A_{p} \not p-B_{p}=\not p-m+-i g^{2} \int \frac{d^{3} l}{(2 \pi)^{3}} \frac{1}{A_{l}^{2} l^{2}-B_{l}^{2}}\left[\Gamma_{B . C}^{\mu}\left(A_{l} l+B_{l}\right) \gamma^{\nu}[l, p] D_{\mu \nu}[p-l]\right]
$$

Finally we reach ${ }^{2}$

\footnotetext{
${ }^{2}$ The corresponding gap equations in the 4 -spinor formalism are identical to those presented here except $\mathcal{F} \rightarrow 0$ and $F \rightarrow 2 F$.
} 


$$
\begin{gathered}
B[p]=m-i g^{2} \int \frac{d^{3} l}{(2 \pi)^{3}} \frac{-1}{A_{l}^{2} l^{2}-B_{l}^{2}}\left[\Omega_{1}\left[B_{l} g^{\mu \nu}+A_{l} l_{\beta}\left(-i \epsilon^{\mu \beta \nu}\right)\right] D_{\mu \nu}+\right. \\
\Omega_{2}\left[B_{l} R^{\mu} R^{\nu}+A_{l} l_{\beta} R^{\mu} R_{\rho}\left(-i \epsilon^{\rho \beta \nu}\right)\right] D_{\mu \nu}+ \\
\left.\Omega_{3} A_{l} l^{\nu} R^{\mu} D_{\mu \nu}[p-l]\right] \\
A[p]=1-i g^{2} \int \frac{d^{3} l}{(2 \pi)^{3}} \frac{-1}{A_{l}^{2} l^{2}-B_{l}^{2}} \frac{-1}{p^{2}}\left\{\Omega_{1}\left[A_{l}\left[P^{\mu} l^{\nu}+P^{\nu} l^{\mu}-(p \cdot l) g^{\mu \nu}\right]-i \epsilon^{\alpha \mu \nu} B_{l} P_{\alpha}\right] D_{\mu \nu}[p-l]+\right. \\
\Omega_{2}\left[A_{l}\left[P^{\mu} R^{\nu}(l \cdot R)+l^{\mu} R^{\nu}(P \cdot R)-R^{\mu} R^{\nu}(p \cdot l)\right]-i \epsilon^{\alpha \rho \nu} R^{\mu} R_{\rho} B_{l} P_{\alpha}\right] D_{\mu \nu}[p-l]+ \\
\left.\Omega_{3}\left[B_{l}\left[R^{\mu} P^{\nu}\right]-i \epsilon^{\alpha \beta \nu} R^{\mu} P_{\alpha} A_{l} l_{\beta}\right] D_{\mu \nu}[p-l]\right\}
\end{gathered}
$$

The full photon propagator takes the form

$$
D_{\mu \nu}=\frac{-1}{\left[k^{2}+F\right]-\frac{\left(\mu_{C S}-\mathcal{F}\right)^{2} k^{2}}{\left[k^{2}+F\right]}}\left[g_{\mu \nu}-\frac{k_{\mu} k_{\nu}}{k^{2}}-i\left(\mu_{C S}-\mathcal{F}\right) \epsilon_{\mu \nu \sigma} \frac{k^{\sigma}}{\left[k^{2}+F\right]}\right]+\xi \frac{k_{\mu} k_{\nu}}{k^{2}} \frac{(-1)}{k^{2}}
$$

Here we allow for an explicit topological photon mass term $\mu_{C S}$ in the propagator. Such a parity-breaking term may be introduced to the theory via a Chern-Simons-like term in the Lagrangian:

$$
\mathcal{L}_{\mathrm{CS}}=\mu_{C S} \frac{1}{4} \epsilon_{\mu \nu \alpha} F^{\mu \nu} A^{\alpha}
$$

Alternatively, the topological photon mass can be dynamically generated. As we shall see, the infrared limit of $\mathcal{F}$ captures the contribution of photon mass from the dynamical fermions. We will go through the derivations of the ring functions $F$ and $\mathcal{F}$ in the full photon propagator in the next section. 


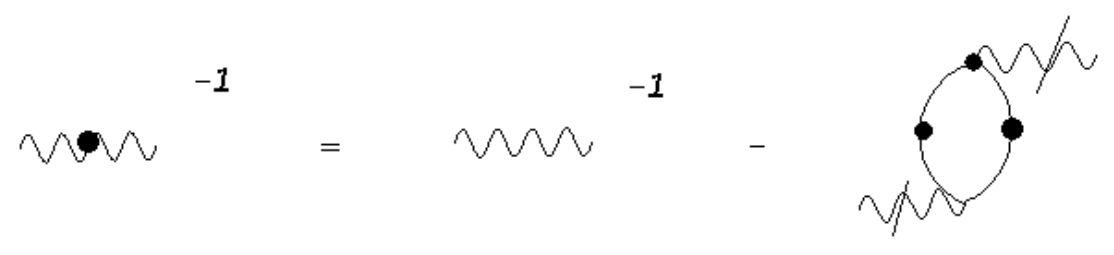

Figure 17: Schwinger-Dyson equations for the photon.

\subsubsection{PHOTON PROPAGATOR}

4.3.3.1 INTRODUCTION We shall consider the following Schwinger Dyson equation for photon:

$$
D_{\mu \nu}^{-1}[k]=D_{\mu \nu}^{(0)}{ }^{-1}[k]+i g^{2} \operatorname{Tr}\left[\left(\Gamma_{B . C}\right)_{\mu} S \gamma_{\nu} S\right]
$$

It is useful to define

$$
\Pi^{\mu \nu}[k]=g^{2} \int \frac{d^{3} l}{(2 \pi)^{3}} \frac{1}{A_{1}^{2} l_{1}^{2}-B_{1}^{2}} \frac{1}{A_{2}^{2} l_{2}^{2}-B_{2}^{2}} \operatorname{Tr}\left[\Gamma_{B . C}^{\mu}[l ; l-k]\left(A_{1} l_{1}+B_{1}\right) \gamma^{\nu}\left(A_{2} l_{2}+B_{2}\right)\right]
$$

where

$$
\begin{aligned}
& l_{1}^{\mu}=l^{\mu} \\
& l_{2}^{\mu}=l^{\mu}-k^{\mu}
\end{aligned}
$$

and we have:

$$
D_{\mu \nu}^{-1}[k]=D_{\mu \nu}^{(0)^{-1}}[k]+i \Pi_{\mu \nu}[k]
$$


4.3.3.2 TENSOR PROPERTIES Now we focus on the various tensor properties of the photon propagator.

Starting with the bare photon propagator

$$
D_{\mu \nu}^{(0)-1}=-k^{2}\left[g_{\mu \nu}-(1-\xi) \frac{k_{\mu} k_{\nu}}{k^{2}}\right]-i \mu_{C S} \epsilon_{\mu \nu \rho} k^{\rho}
$$

inverting gives

$$
D_{\mu \nu}^{(0)}=\frac{-1}{k^{2}-\mu_{C S}^{2}}\left[g_{\mu \nu}-\frac{k_{\mu} k_{\nu}}{k^{2}}-i \mu_{C S} \epsilon_{\mu \nu \rho} k^{\rho}\right]+\xi \frac{k_{\mu} k_{\nu}}{k^{2}} \frac{(-1)}{k^{2}} .
$$

For the full photon gap equation:

$$
D_{\mu \nu}{ }^{-1}=D_{\mu \nu}^{(0)}{ }^{-1}+i \Pi_{\mu \nu}
$$

with

$$
\Pi^{\mu \nu}[k]=g^{2} \int \frac{d^{3} l}{(2 \pi)^{3}} \frac{1}{A_{1}^{2} l_{1}^{2}-B_{1}^{2}} \frac{1}{A_{2}^{2} l_{2}^{2}-B_{2}^{2}} \operatorname{Tr}\left[\Gamma_{B . C}^{\mu}[l ; l-k]\left(A_{1} l_{1}+B_{1}\right) \gamma^{\nu}\left(A_{2} l_{2}+B_{2}\right)\right]
$$

where

$$
\begin{aligned}
& l_{1}^{\mu}=l^{\mu} \\
& l_{2}^{\mu}=l^{\mu}-k^{\mu} .
\end{aligned}
$$

We define:

$$
i \Pi_{\mu \nu}=-\left[g_{\mu \nu}-\frac{k_{\mu} k_{\nu}}{k^{2}}\right] F+i \epsilon_{\mu \nu \rho} k^{\rho} \mathcal{F}
$$

Here, $F$ is the usual polarization scalar, while the existence of $\mathcal{F}$ originates from the trace $\operatorname{Tr}[\gamma \gamma \gamma]$. 
It is useful to work out:

$$
\begin{gathered}
F[k]=-\frac{1}{2}\left[g_{\mu \nu}-3 \frac{k_{\mu} k_{\nu}}{k^{2}}\right] i \Pi^{\mu \nu} \\
\mathcal{F}[k]=\frac{1}{2 k^{2}} \epsilon^{\mu \nu \rho} k_{\rho} \Pi_{\mu \nu}
\end{gathered}
$$

Hence, the Schwinger-Dyson equation for the photon propagator can be rewritten as

$$
D_{\mu \nu}^{-1}=-k^{2}\left[g_{\mu \nu}-(1-\xi) \frac{k_{\mu} k_{\nu}}{k^{2}}\right]-i \mu_{C S} \epsilon_{\mu \nu \sigma} k^{\sigma}+-\left[g_{\mu \nu}-\frac{k_{\mu} k_{\nu}}{k^{2}}\right] F+i \epsilon_{\mu \nu \rho} k^{\rho} \mathcal{F}
$$

Inverting the above gives:

$$
D_{\mu \nu}=\frac{-1}{\left[k^{2}+F\right]-\frac{\left(\mu_{C S}-\mathcal{F}\right)^{2} k^{2}}{\left[k^{2}+F\right]}}\left[g_{\mu \nu}-\frac{k_{\mu} k_{\nu}}{k^{2}}-i\left(\mu_{C S}-\mathcal{F}\right) \epsilon_{\mu \nu \sigma} \frac{k^{\sigma}}{\left[k^{2}+F\right]}\right]+\xi \frac{k_{\mu} k_{\nu}}{k^{2}} \frac{(-1)}{k^{2}}
$$

$F[k]$ thus defined is rendered finite by projection: the UV divergence in $\Pi_{\mu \nu}$ is automatically projected out. In fact, gauge invariance ensures $k_{\mu} \Pi^{\mu \nu}=0$, and hence all projections

$$
F[k]=-\frac{1}{2}\left[g_{\mu \nu}-x \frac{k_{\mu} k_{\nu}}{k^{2}}\right] i \Pi_{\mu \nu}
$$

are equivalent for any choice of $x^{3}[44]$.

On the other hand, $\mathcal{F}$ plays the role of a topological photon mass (similar to the bare breaking term $\mu_{C S}$ ), and is dynamically generated in the 2-spinor formalism.

Now, plugging in the Ansätz of the fermionic propagator in equation 4.7, we obtain the final form of the ring functions $F$ and $\mathcal{F}$ :

$$
F=-i \frac{1}{2} g^{2} \int \frac{d^{3} l}{(2 \pi)^{3}} \frac{1}{A_{1}^{2} l_{1}^{2}-B_{1}^{2}} \frac{1}{A_{2}^{2} l_{2}^{2}-B_{2}^{2}}(\operatorname{Tr} I)\left[\tilde{\Omega}_{F 1}+\tilde{\Omega}_{F 2}+\tilde{\Omega}_{F 3}\right]
$$

\footnotetext{
${ }^{3}$ This is not true if Ward identity is violated, for example, employing the bare vertex as in the rainbow ladder approximation with the full fermion propagator. For RL, one needs to absorb the violating piece (fixing $x=3$ ) into the definition of the truncation.
} 


$$
\begin{gathered}
\tilde{\Omega}_{F 1}=\Omega_{1} 2 A_{1} A_{2}\left[l_{1} \cdot l_{2}-3 \frac{\left(k \cdot l_{1}\right)\left(k \cdot l_{2}\right)}{k^{2}}\right] \\
\tilde{\Omega}_{F 2}=\Omega_{2}\left[A _ { 1 } A _ { 2 } \left\{\left[2\left(l_{1} \cdot R\right)\left(l_{2} \cdot R\right)-\left(l_{1} \cdot l_{2}\right) R^{2}\right]+\right.\right. \\
\left.-\frac{3}{k^{2}}(R \cdot k)\left[\left(l_{1} \cdot k\right)\left(l_{2} \cdot R\right)+\left(l_{2} \cdot k\right)\left(l_{1} \cdot R\right)-(R \cdot k)\left(l_{1} \cdot l_{2}\right)\right]\right\}+ \\
\left.B_{1} B_{2}\left[R^{2}-3 \frac{(k \cdot R)^{2}}{k^{2}}\right]\right] \\
\tilde{\Omega}_{F 3}=\Omega_{3}\left[A_{1} B_{2}\left[\left(l_{1} \cdot R\right)-3 \frac{\left(k \cdot l_{1}\right)(k \cdot R)}{k^{2}}\right]+A_{2} B_{1}\left[\left(l_{2} \cdot R\right)-3 \frac{\left(k \cdot l_{2}\right)(k \cdot R)}{k^{2}}\right]\right. \\
R^{\mu}=2 l^{\mu}-k^{\mu} \\
l_{1}^{\mu}=l^{\mu} \\
l_{2}^{\mu}=l^{\mu}-k^{\mu} \\
\Omega_{1}=\frac{A_{2}+A_{1}}{2} \\
\Omega_{2}=\frac{1}{l_{2}^{2}-l_{1}^{2}} \frac{A_{2}-A_{1}}{2} \\
\Omega_{3}=-\frac{1}{l_{2}^{2}-l_{1}^{2}}\left(B_{2}-B_{1}\right) \\
\Omega^{\mu}
\end{gathered}
$$

and similarly

$$
\mathcal{F}=i \frac{1}{2 k^{2}} g^{2} \int \frac{d^{3} l}{(2 \pi)^{3}} \frac{1}{A_{1}^{2} l_{1}^{2}-B_{1}^{2}} \frac{1}{A_{2}^{2} l_{2}^{2}-B_{2}^{2}}(\operatorname{Tr} I)\left[\tilde{\Omega}_{\mathcal{F} 1}+\tilde{\Omega}_{\mathcal{F} 2}+\tilde{\Omega}_{\mathcal{F} 3}\right]
$$

$$
\begin{aligned}
& \tilde{\Omega}_{\mathcal{F} 1}=2 \Omega_{1}\left[A_{1} B_{2} l_{1} \cdot k-A_{2} B_{1} l_{2} \cdot k\right] \\
& \tilde{\Omega}_{\mathcal{F} 2}=\Omega_{2}\left\{A_{1} B_{2}\left[R^{2}\left(k \cdot l_{1}\right)-\left(R \cdot l_{1}\right)(R \cdot k)\right]-A_{2} B_{1}\left[R^{2}\left(k \cdot l_{2}\right)-\left(R \cdot l_{2}\right)(R \cdot k)\right]\right\} \\
& \tilde{\Omega}_{\mathcal{F} 3}=\Omega_{3} A_{1} A_{2}\left[\left(k \cdot l_{1}\right)\left(R \cdot l_{2}\right)-\left(k \cdot l_{2}\right)\left(R \cdot l_{1}\right)\right] \\
& R^{\mu}=2 l^{\mu}-k^{\mu} \\
& l_{1}{ }^{\mu}=l^{\mu} \\
& l_{2}{ }^{\mu}=l^{\mu}-k^{\mu} \\
& \Omega_{1}=\frac{A_{2}+A_{1}}{2} \\
& \Omega_{2}=\frac{1}{l_{2}^{2}-l_{1}^{2}} \frac{A_{2}-A_{1}}{2} \\
& \Omega_{3}=-\frac{1}{l_{2}^{2}-l_{1}^{2}}\left(B_{2}-B_{1}\right)
\end{aligned}
$$




\subsection{PERTURBATIVE LIMIT OF THE POLARIZATION SCALARS}

It is a useful exercise to study the perturbative expression of the two polarization scalars generated dynamically in one loop calculation of the photon propagator.

$$
\Pi^{\mu \nu}[k]=g^{2} \int \frac{d^{3} l}{(2 \pi)^{3}} \frac{1}{A_{1}^{2} l_{1}^{2}-B_{1}^{2}} \frac{1}{A_{2}^{2} l_{2}^{2}-B_{2}^{2}} \operatorname{Tr}\left[\Gamma^{\mu}[l ; l-k]\left(A_{1} l_{1}+B_{1}\right) \gamma^{\nu}\left(A_{2} l_{2}+B_{2}\right)\right]
$$

where

$$
\begin{aligned}
& l_{1}^{\mu}=l^{\mu} \\
& l_{2}^{\mu}=l^{\mu}-k^{\mu}
\end{aligned}
$$

and we define:

$$
i \Pi_{\mu \nu}=-\left[g_{\mu \nu}-\frac{k_{\mu} k_{\nu}}{k^{2}}\right] F+i \epsilon_{\mu \nu \rho} k^{\rho} \mathcal{F}
$$

hence:

$$
\begin{gathered}
F[k]=-\frac{1}{2}\left[g_{\mu \nu}-3 \frac{k_{\mu} k_{\nu}}{k^{2}}\right] i \Pi^{\mu \nu} \\
\mathcal{F}[k]=\frac{1}{2 k^{2}} \epsilon^{\mu \nu \rho} k_{\rho} \Pi_{\mu \nu}
\end{gathered}
$$

The perturbative limit refers to:

$$
F=-i \frac{1}{2} g^{2} \int \frac{d^{3} l}{(2 \pi)^{3}} \frac{1}{A_{1}^{2} l_{1}^{2}-B_{1}^{2}} \frac{1}{A_{2}^{2} l_{2}^{2}-B_{2}^{2}}(\operatorname{Tr} I)\left[\tilde{\Omega}_{F 1}+\tilde{\Omega}_{F 2}+\tilde{\Omega}_{F 3}\right]
$$




$$
\begin{aligned}
\tilde{\Omega}_{F 1} & =2 A_{1} A_{2}\left[l_{1} \cdot l_{2}-3 \frac{\left(k \cdot l_{1}\right)\left(k \cdot l_{2}\right)}{k^{2}}\right] \\
\tilde{\Omega}_{F 2} & =0 . \\
\tilde{\Omega}_{F 3} & =0 . \\
A & =1 \\
B & =M
\end{aligned}
$$

and similarly for $\mathcal{F}$

$$
\begin{aligned}
\mathcal{F}=i \frac{1}{2 k^{2}} g^{2} \int \frac{d^{3} l}{(2 \pi)^{3}} \frac{1}{A_{1}^{2} l_{1}^{2}-B_{1}^{2}} \frac{1}{A_{2}^{2} l_{2}^{2}-B_{2}^{2}}(\operatorname{Tr} I)\left[\tilde{\Omega}_{\mathcal{F} 1}+\tilde{\Omega}_{\mathcal{F} 2}+\tilde{\chi}_{\mathcal{F} 2}+\tilde{\Omega}_{\mathcal{F} 3}\right] \\
\tilde{\Omega}_{\mathcal{F} 1}=2\left[A_{1} B_{2} l_{1} \cdot k-A_{2} B_{1} l_{2} \cdot k\right] \\
\tilde{\Omega}_{\mathcal{F} 2}=0 . \\
\tilde{\Omega}_{\mathcal{F} 3}=0 . \\
A=1 \\
B=M .
\end{aligned}
$$

Performing the integral using Feynman trick would result:

$$
\begin{aligned}
& F[k]=-2 \alpha \int_{0}^{1} d x \frac{x(1-x) k_{E}^{2}}{\sqrt{M^{2}+x(1-x) k_{E}^{2}}} \\
& \mathcal{F}[k]=-\alpha M \int_{0}^{1} d x \frac{1}{\sqrt{M^{2}+x(1-x) k_{E}^{2}}}
\end{aligned}
$$

In particular, in the limit of $k^{2} \rightarrow 0$ :

$$
\begin{aligned}
\lim _{k^{2} \rightarrow 0} F[k] / k^{2} & =-\frac{\alpha}{3 M} \\
\lim _{k^{2} \rightarrow 0} \mathcal{F}[k] & =-\alpha \frac{M}{|M|}
\end{aligned}
$$




\subsection{COLEMAN-HILL THEOREM}

We see in section 4.4 that the one-loop perturbative expression of $\mathcal{F}$ is given by

$$
\lim _{k^{2} \rightarrow 0} \mathcal{F}[k]=-\alpha \frac{M}{|M|} .
$$

In the chiral limit $\left(M \longrightarrow 0^{+}\right)$:

$$
\lim _{k^{2} \rightarrow 0} \mathcal{F}[k]=-\alpha .
$$

The Coleman-Hill theorem [42] states that the above result is exact, i.e the topological photon mass will receive no contribution from higher loop diagrams nor from the dressing of fermionic propagator. The theorem is based on gauge invariance and power counting, here we briefly recount the main ideas in the proof.

Consider the effective action of the electromagnetic fields, obtained by integrating over the fermion field. We denote the resulting n-point vertex function (Lorentz indices suppressed)

$$
\begin{array}{r}
\Gamma^{(n)}\left[p_{1}, p_{2}, \ldots, p_{n}\right] \\
p_{n}=-\sum_{j=1}^{n-1} p_{j}
\end{array}
$$

with the delta function for the conservation of momentum factored out.

Gauge invariance requires

$$
p_{1} \cdot \Gamma^{(n)}\left[p_{1}, p_{2}, \ldots, p_{n}\right]=0,
$$

then we obtain (by differentiating $p_{1}$ on both sides)

$$
\Gamma^{(n)}\left[p_{1}, p_{2}, \ldots, p_{n}\right]+p_{1} \cdot \partial_{p_{1}} \Gamma^{(n)}=0 .
$$


Hence, we observe that $\Gamma^{(n)}\left[p_{1} \rightarrow 0, \ldots\right]=0$, or

$$
\Gamma^{(n)}\left[p_{1}, p_{2}, \ldots, p_{n}\right]=O\left[p_{1}\right] .
$$

The same argument holds true for $p_{j}$ for $j=1,2, \ldots, n-1$, in particular, we note

$$
\begin{array}{r}
\Gamma^{(n)}\left[p_{1}, p_{2}, \ldots, p_{n}\right]=O\left[p_{1} p_{2}\right] \\
n>2
\end{array}
$$

We shall focus on the photon self-energy term $\mathcal{F}[0]$. Two external legs of momentum $\pm k$ should be connected to the general n-point functions, for simplicity, we will pick them to be $p_{1}$ and $p_{2}$. The key argument for the Coleman-Hill theorem is that:

- if the two external lines land on two separate fermion loops, from equation 4.36, one concludes the contribution is $O\left[k^{2}\right]$,

- if the two external lines land on the same fermion loop, from equation 4.37, one concludes again that the contribution is $O\left[k^{2}\right]$, except when $\mathbf{n}=\mathbf{2}$.

Given $(k) \mathcal{F}[k] \approx O[k]$ for small $k$, any contribution that is of order $O\left[k^{2}\right]$ cannot contribute to it. We conclude that the only contribution to $\mathcal{F}[0]$ is from bare $n=2$ loop diagram: the fermion loop with no photon line attached, i.e no dressing. This finishes the proof of the Coleman-Hill theorem.

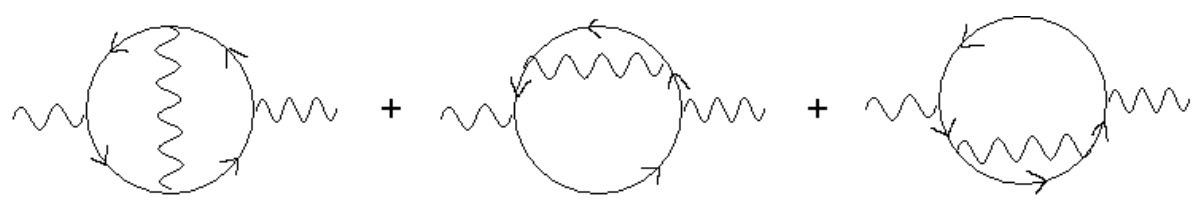

Figure 18: Two-loop corrections to $\mathcal{F}[k]$. By Coleman-Hill theorem, the sum of their contributions to $\mathcal{F}[0]$ should be exactly zero. 
On passing, we note that an arbitrary truncation of the Schwinger-Dyson equations will not be consistent with the Coleman-Hill theorem. However, it would serve as a good test to gauge how good a given truncation scheme is.

\subsection{NUMERICS AND RESULTS}

We shall present the results for the dynamical parity and chiral symmetry breaking in QED3. For our numerical study, we shall work exclusively in Euclidean space ${ }^{4}$. The order parameter for chiral symmetry breaking is the condensate, which is given by

$$
\langle\bar{\psi} \psi\rangle=2 \int \frac{d^{3} k_{E}}{2 \pi)^{3}} \frac{B}{A^{2} k_{E}^{2}+B^{2}} .
$$

One can equally well pick $B(0)$ as the order parameter, in fact, inspection of equation 4.8 reveals their relation:

$$
B\left(p_{E}^{2}\right) \longrightarrow 2 \pi \alpha \frac{2+\xi}{p_{E}^{2}}\langle\bar{\psi} \psi\rangle
$$

for large $p_{E}^{2}$. This result holds true regardless of the choice of vertex approximation and the same expression can be derived with operator product expansion [45].

\subsubsection{STRATEGY OF SOLVING SDES}

We summarize the major steps in solving the SDEs for the current problem:

- Firstly, one derives the hierarchy of SDEs.

- Then, one decides on a truncation scheme, e.g Ward identity preserving vertex $\Gamma_{B . C}$. The vertex can usually be written as a functional of the fermion propagator: $\Gamma[S]$.

\footnotetext{
${ }^{4}$ For the convention of the transformations between Minkowski and Euclidean spaces, see appendix A
} 
- With the truncation, the photon propagator can also be written as a functional of the fermion propagator $(\Pi[S])$. At this point, one can solve the gap equations in terms of fermion propagator only. Non-perturbative calculation then becomes a self-consistent calculation.

- One obtains the various interesting quantities, such as the condensate.

- The accuracy of the truncation scheme can be checked with Coleman-Hill theorem.

We shall employ a variety of truncation schemes in the study: the rainbow ladder approximation (RL), the central Ball-Chu vertex (CBC), the Ball-Chu vertex (BC), the Ball-Chu with the Curtis-Pennington vertex $(\mathrm{CP})[46,47]$. Their definitions are listed below:

$$
\begin{aligned}
\Gamma_{R L}^{\nu}[p, q]= & \gamma^{\nu} \\
\Gamma_{C B C}^{\nu}[p, q]= & \frac{A_{q}+A_{p}}{2} \gamma^{\nu} \\
\Gamma_{B C}^{\nu}[p, q]= & \frac{A_{q}+A_{p}}{2} \gamma^{\nu}+\frac{(q+p)^{\nu}}{q^{2}-p^{2}}\left[\left(A_{q}-A_{p}\right) \frac{(q+p)_{\rho} \gamma^{\rho}}{2}-\left(B_{q}-B_{p}\right)\right] \\
\Gamma_{C P}^{\nu}[p, q]= & \Gamma_{B C}^{\nu}[p, q]+\frac{A_{q}-A_{p}}{2} \frac{q^{2}+p^{2}}{\left(q^{2}-p^{2}\right)^{2}+\left[M_{q}^{2}+M_{p}^{2}\right]^{2}} \\
& {\left[\left(q^{2}-p^{2}\right) \gamma^{\nu}-(\not q-\not p)(q+p)^{\nu}\right] . }
\end{aligned}
$$

The definition of CP vertex is motivated by multiplicative renormalization for fermions, hence we shall only apply the vertex to the fermionic gap equations but not the photonic one.

Finally, we choose to work in Landau gauge as it is particularly reliable for the class of truncations considered here [48, 49].

\subsubsection{PARITY SYMMETRY BREAKING}

To study parity symmetry breaking, we classify the fermions according to the sign of their mass functions $M$. It is useful to introduce the asymmetry parameter

$$
\eta=\frac{N_{+}-N_{-}}{N_{+}+N_{-}}
$$


where $N_{+}$is the number of fermions with mass $M$ while $N_{-}$is that for mass $-M$. At $\eta=0$, one obtains a parity-preserving vacuum: a system with $\frac{N_{f}}{2}$ fermions of mass $M$, and an equal number of fermions with mass $-M$. For $\eta=1$, parity is maximally violated $\left(N_{f}\right.$ fermions of the same type).

Presenting below is the graph of $B(0)$ versus $\eta$ for RL and CBC truncations:

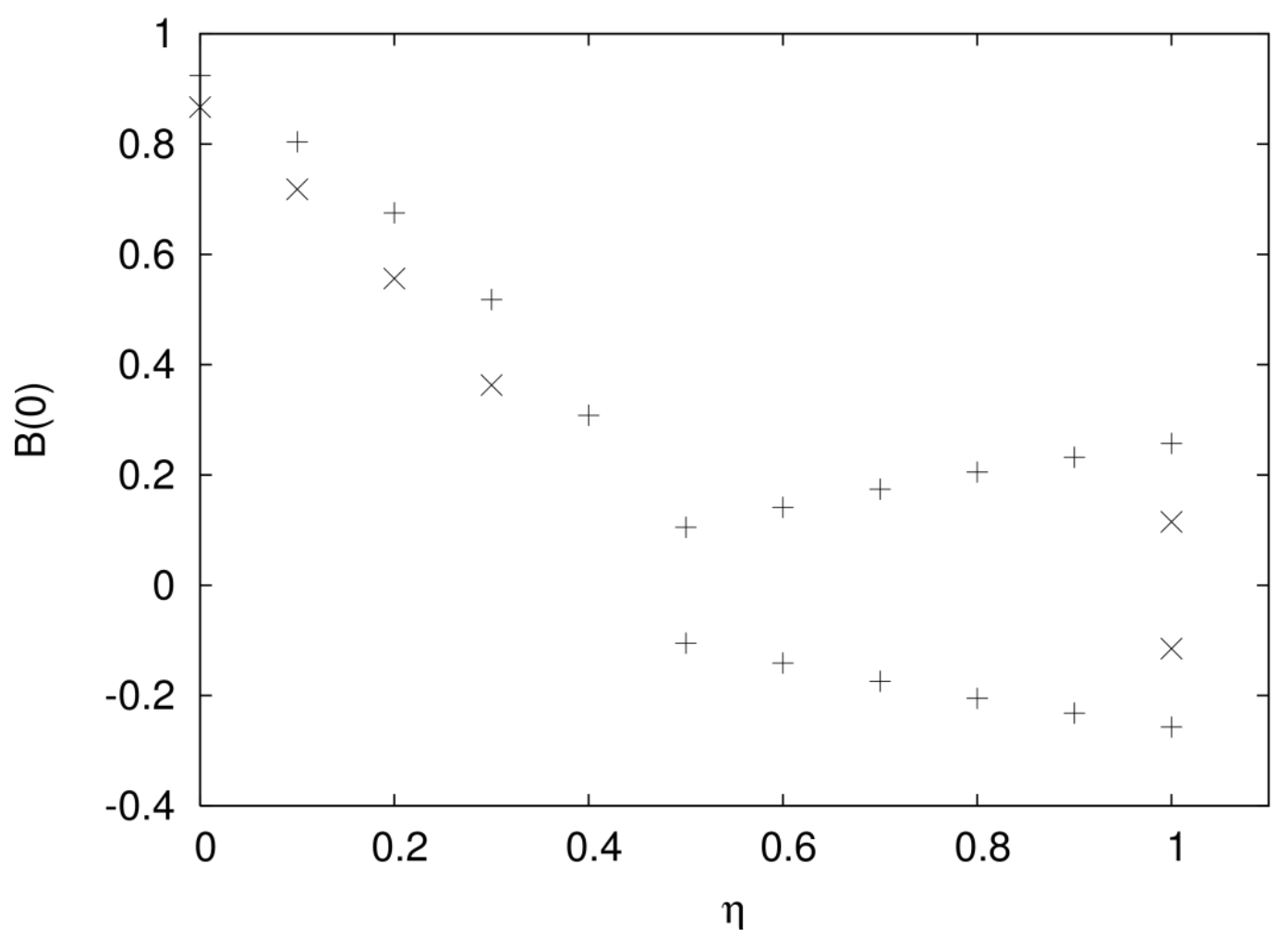

Figure 19: Parity-breaking solution vs $\eta$. Pluses are RL and Xs are CBC.

Here we record the most important observations:

- For $\eta<0.4$, stable iterative solutions exist for RL truncation.

- With $\eta \gtrsim 0.4$ within RL scheme, the iterative method yields a limit cycle: the iteration oscillates between $(B, A) \leftrightarrow(-B, A)$. However on closer investigation with other numerical methods such as minimization, only trivial solution $(B=0)$ can be found, suggesting that the cycle itself is not a solution of SDEs. 
- Similar behavior is found for $\mathrm{CBC}$ truncation, except that the transition asymmetry becomes $\eta=0.35$. However, between $\eta=0.35$ to $\eta=1$, the iterative approach displays chaotic behaviors, meaning there is no solution for SDEs.

\subsubsection{CHIRAL SYMMETRY BREAKING}

Now we turn to the study of chiral symmetry breaking. In this case, we consider $\eta=0$, i.e $\frac{N_{f}}{2}$ fermions in each of the $\pm M$ states. The solutions of the Schwinger-Dyson equations under various truncation schemes are collected:

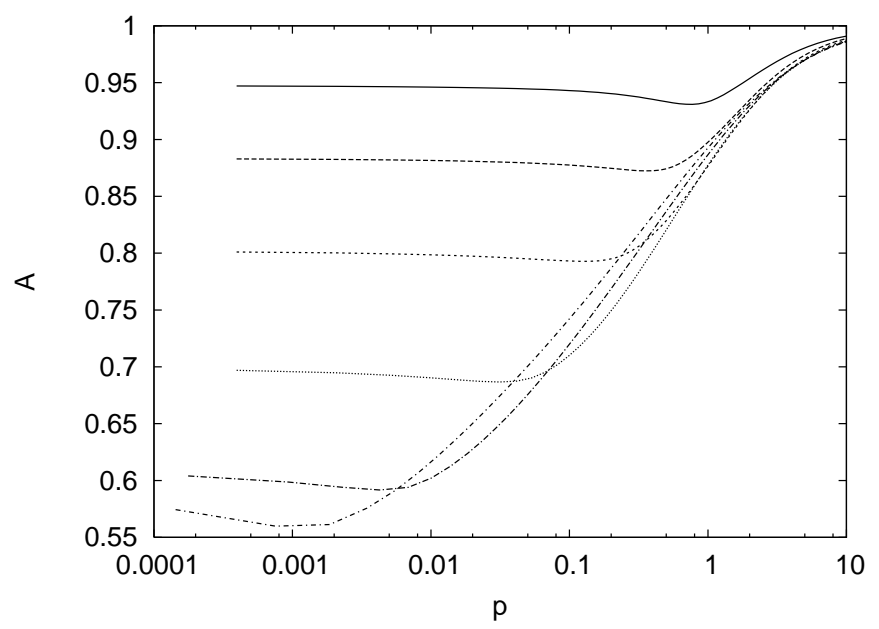

Figure 20: $A$ vs. $p$ for various $N_{f}(\mathrm{~L})$. These curves are obtained in the CBC truncation and correspond to $N_{f}=1,2,3,4,5$, and 5.75 from top to bottom. 


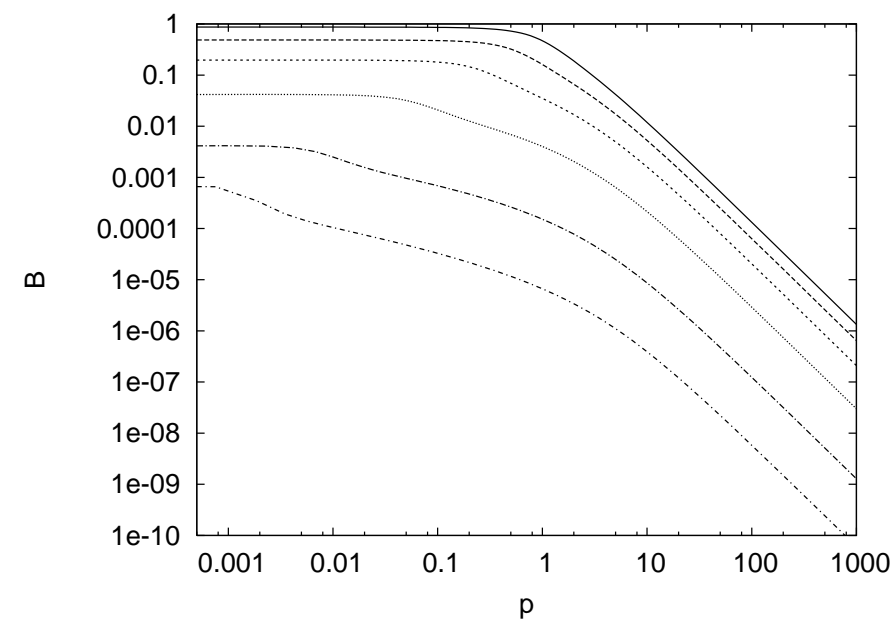

Figure 21: $B$ vs. $p$ for various $N_{f}(\mathrm{R})$. These curves are obtained in the CBC truncation and correspond to $N_{f}=1,2,3,4,5$, and 5.75 from top to bottom.

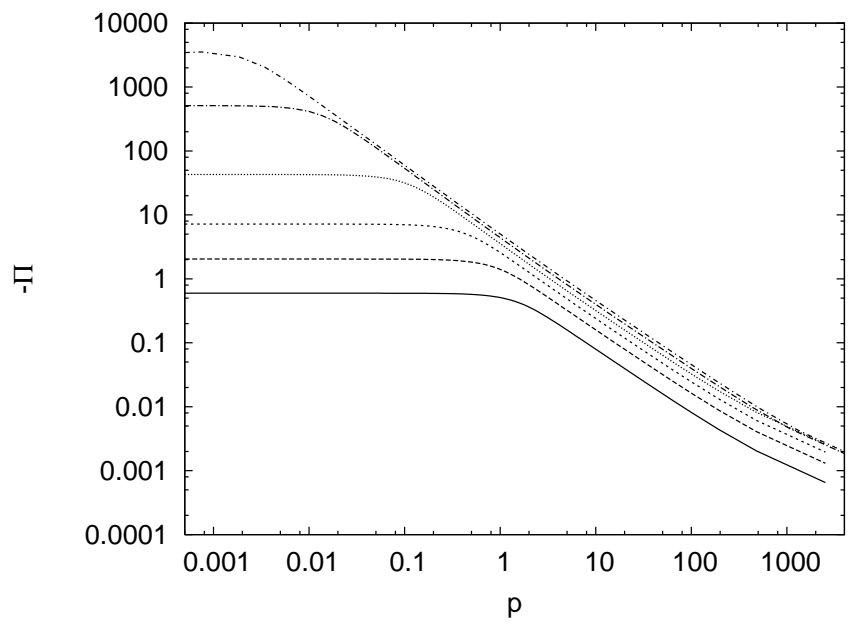

Figure 22: Vacuum polarization $\left(\Pi=\frac{F}{k^{2}}\right)$ vs. momentum for various $N_{f}$. These curves are obtained in the CBC truncation and correspond to $N_{f}=1,2,3,4,5$, and 5.75 from bottom to top. 
The study of large $N_{f}$ QED3 by Appelquist [41] motivates the following behavior of $B(0)$ :

$$
B(0) \propto N_{f} e^{\frac{-2 \pi}{\sqrt{N_{\star}^{A} / N_{f}-1}}}
$$

with $N_{\star}^{A}=\frac{64}{\pi^{2}}$.

We shall fit the condensates obtained in various truncations with the following Ansätz:

$$
\langle\bar{\psi} \psi\rangle\left(N_{f}\right)=a N_{f} e^{\frac{-2 \pi}{\sqrt{N_{\star} / N_{f}-1}}}
$$

The best-fit parameters are found to be:

$$
\begin{aligned}
a & =1.8 \\
N_{\star}[R L] & =1.10 N_{\star}^{A} \\
N_{\star}[C B C] & =1.00 N_{\star}^{A} \\
N_{\star}[B C] & =N_{\star}[C P]=1.21 N_{\star}^{A} \\
N_{\star}^{A}=\frac{64}{\pi^{2}} &
\end{aligned}
$$




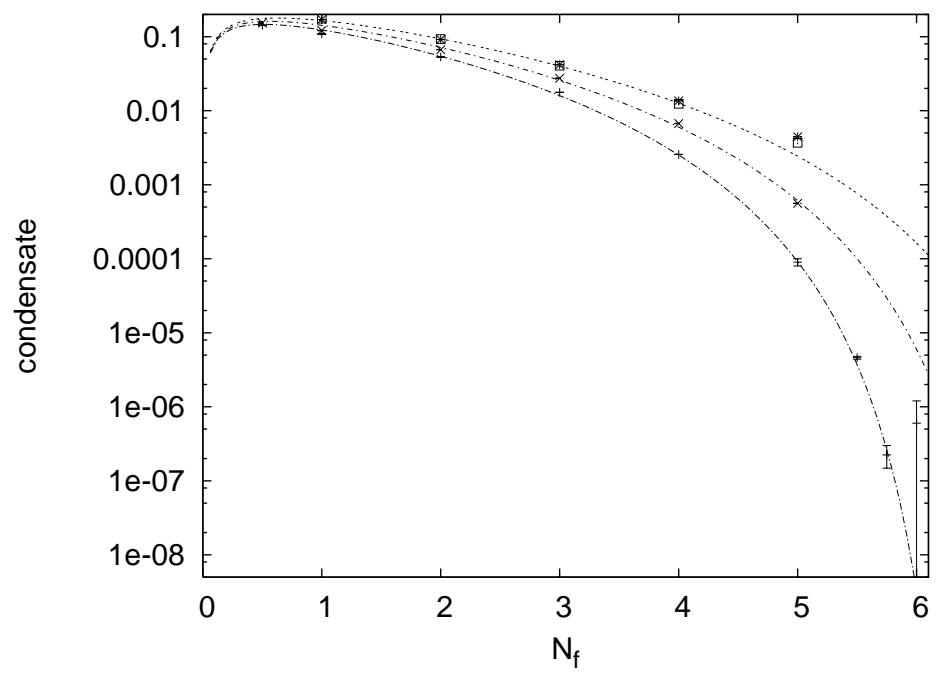

Figure 23: The condensate for (top to bottom) BC, CP, RL, and CBC models, with fit functions (described in the text).

We remark on two important aspects of the chiral symmetry breaking in QED3.

Firstly, a large ratio of scales is dynamically generated as $N_{f}$ changes. This property is an important clue for the construction of BSM models in order to explain the large mass hierarchies of the Standard Model fermions. In fact, the mass drops from $O(\alpha)$ at $N_{f}=1$ to $10^{-8} \alpha$ at $N_{f}=6$. This becomes the major hindrance for the lattice computation as extremely large lattices is needed to achieve the continuum limit, especially for low mass scale.

Secondly, we observe that generally there is a rapid drop in the value of condensate near $N_{\star}$. However, the order parameter may or may not reach zero, in the case it does, we have a genuine phase transition: a chiral restoration; in the case it does not, the condensate is only exponentially suppressed [50]: we have a crossover. We shall briefly discuss the condition of the two scenarios in the next section. 


\subsubsection{NOTES ON CHIRAL PHASE RESTORATION QED3 is confining in}

the sense that the quenched theory has a non-zero string tension [51], this property persists if the photon is dressed by massive fermions. This fact can be illustrated easily with a perturbative calculation.

Firstly, we consider the quenched case. For Landau gauge, the time component of bare photon propagator reads

$$
D_{00}\left[k^{0}=0, \vec{k}\right]=\frac{1}{\vec{k}^{2}}
$$

The corresponding potential in configuration space is defined as

$$
\begin{aligned}
V(r) & =g^{2} \int \frac{d^{2} k}{(2 \pi)^{2}} e^{-i \vec{k} \cdot \vec{r}} \frac{1}{\overrightarrow{k^{2}}} \\
& =\frac{\alpha}{\pi} \int d k k \frac{1}{k^{2}} 2 \pi j_{0}[k r]
\end{aligned}
$$

This integral is infrared divergent ${ }^{5}$, instead we consider

$$
\frac{d V(r)}{d r}=\frac{\alpha}{\pi} \int d k d \phi(k)(-i k \cos [\phi]) \frac{1}{k^{2}} e^{-i k r \cos [\phi]},
$$

\footnotetext{
${ }^{5}$ If we pick the following regularization scheme:

$$
V(r)=\frac{\alpha}{\pi} \int d k \frac{1}{k+\lambda} 2 \pi j_{0}[k r]
$$

Formally, we have:

$$
\int d k \frac{1}{k+\lambda} j_{0}[k r] \rightarrow-j_{0}[r \lambda]\left[\ln \left[\frac{r}{2}\right]+\ln [\lambda]\right]+\frac{\pi}{2} H_{0}[\lambda r]-F^{1,0}\left[1,-\frac{1}{4} r^{2} \lambda^{2}\right]
$$

where $H_{0}$ is the Struve function of the zeroth order, $F$ is the regularized hypergeometric function.

We expand in order of $\lambda$ and obtain $\bar{V}(r)=V(r)-V\left(r_{0}\right)=-2 \alpha \ln r+\ldots$ We can easily pick another regularization scheme:

$$
V(r)=\frac{\alpha}{\pi} \int d k k \frac{1}{k^{2}+\lambda^{2}} 2 \pi j_{0}[k r]
$$

The integral is formally $V(r)=\frac{\alpha}{\pi} \int d k k_{\frac{1}{k^{2}+\lambda^{2}}} 2 \pi j_{0}[k r]=2 \alpha K_{0}[\lambda r]$, where $K$ is the modified Bessel function of the second kind. Expanding in order of $\lambda$ again gives $V(r)=-2 \alpha \ln r+\ldots$. As expected, the definition of $\bar{V}$ is regularization independent.
} 
the integral is then perfectly finite and is given by

$$
\frac{d V(r)}{d r} \rightarrow \frac{-2 \alpha}{r}
$$

and we obtain the logarithmic confining potential in the quenched case:

$$
\bar{V}[r]=-2 \alpha \ln [\alpha r]+C
$$

The theory loses its confining property if the potential is dressed by massless fermions, as we will now illustrate.

The perturbative expression of $F$ for massless fermions is given by

$$
F_{M=0}^{P}=-\alpha \frac{\pi}{4} k
$$

The potential becomes:

$$
V(r) \rightarrow \frac{\alpha}{\pi} \int d k k \frac{1}{k^{2}+\alpha \frac{\pi}{4} k} 2 \pi j_{0}[k r]
$$

The integral in this case is finite.

For long distance behavior, we focus on the small $k$ region. Observe that the polarization term dominates and the large $r$ behavior is of the form $\frac{1}{r}$. (formally the $\alpha$ cancels and $\left.V \rightarrow \frac{8}{\pi r}\right)$

If we are interested in the small $r$ region, we need to look at the large $k$ behavior, which is the original bare photon $k^{2}$ piece. The resultant configuration space potential of course behaves as $-\ln [r]$. Note that $\alpha$ is the only scale in the problem, and hence small $r$ and large $r$ refers to $r \alpha<<1$ and $r \alpha>>1$ respectively.

Hence we see that massless fermions in the theory completely screen the confining potential ${ }^{6}$.

\footnotetext{
${ }^{6}$ Formally the integral is given by
} 
However, if the fermions are massive, the confining properties are restored. Consider the perturbative polarization for massive fermions:

$$
F_{M}^{P}=-2 \alpha \int_{0}^{1} d x \frac{x(1-x) k^{2}}{\sqrt{M^{2}+x(1-x) k^{2}}}
$$

Inspecting of the large $k$ (small $r$ ) behavior of the above expression, we can immediately infer that the potential is confining, only that the effective coupling is different by a factor $\frac{1}{1+\frac{\alpha}{3 M}}$ for the present case.

Hence, the massive fermion loops, in the infrared limit, serve only to rescale $\alpha$, and the potential remains confining.

The key idea is that whether the theory is confining depends crucially on the infrared behavior of the vacuum polarization, more precisely, the limit of $\frac{F}{k^{2}}$ : if it's finite (dressed with massive fermions) or zero (quenched calculation), the theory is confining; if it tends to infinity (dressed by massless fermions), the theory is not confining.

For a confining theory, it can be argued that once DCSB occurs for one $N_{f}$, it occurs for all $N_{f}$, as the changing of number of flavors amounts to the rescaling of the coupling. It follows that no chiral phase restoration will occur. The mass is only exponentially suppressed, as suggested by some authors [50]. However, Fischer et al. and Maris [46, 44] suggested the feedback from the fermion propagator functions onto the vacuum polarization can be important. By employing the full fermion propagator in the symmetric phase, i.e massless propagators, in the dressing of the ring, phase restoration can be recovered. Our numerical study generally agrees with this observation, and our results on the critical number of flavors are fairly close to those obtained by the latter authors ${ }^{7}$.

$$
\int d k \frac{1}{k+\lambda} j_{0}[k r] \rightarrow-j_{0}[r \lambda]\left[\ln \left[\frac{r}{2}\right]+\ln [\lambda]\right]+\frac{\pi}{2} H_{0}[\lambda r]-F^{1,0}\left[1,-\frac{1}{4} r^{2} \lambda^{2}\right]
$$

where $\lambda=\frac{\alpha \pi}{4}, H_{0}$ is the Struve function of the zeroth order, $F$ is the regularized hypergeometric function. The expansions in $\lambda r$ and $\frac{1}{\lambda r}$ give the two limits described.

${ }^{7}$ Observe how the infrared limit of $\frac{F}{k^{2}}$ develops into infinity as we approach the critical number of flavors in Figure 22. 


\subsubsection{THE COLEMAN-HILL CONSTRAINT}

Last, but not least, we look at the Coleman-Hill constraint on the various truncation. Recall that the Coleman-Hill theorem is motivated as a diagnostic for truncation accuracy. As the constraint is based on gauge invariance, it is reasonable to expect that a Ward identity preserving truncation (such as $\mathrm{BC}$ and $\mathrm{CP}$ ) will perform better than an arbitrary truncation (such as RL). The result below shows that it is indeed the case:

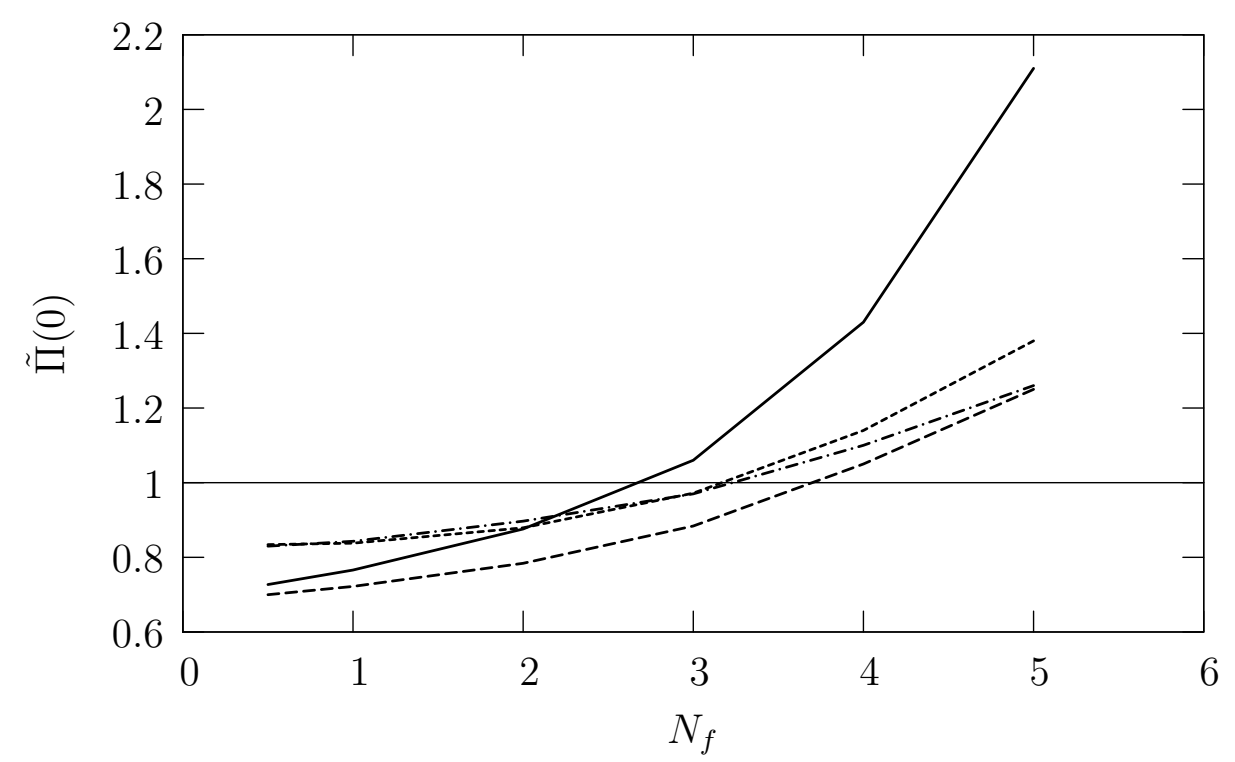

Figure 24: $\tilde{\Pi}(0)$ vs. $N_{f}$ for various models. At $N_{f}=5$, from top to bottom, these are: RL, $\mathrm{CBC}, \mathrm{CP}, \mathrm{BC}$.

\subsubsection{NUMERICAL ASIDES}

4.6.5.1 INTERPOLATOR The numerical study presented here is performed by discretizing the Schwinger-Dyson equations on a momentum grids. The occurrences of the "off-grids" quantities in the integral equations necessitates the use of interpolators. It is essential to minimize the interpolator dependence for the solutions obtained.

As an illustration, we consider the computation of ring function $F[k]$ in the rainbow ladder truncation scheme, for a given model fermion propagator: 


$$
\begin{aligned}
& B[k]=\frac{0.5}{1+k^{2}} \\
& A[k]=1-\frac{0.3}{1+k^{2}} .
\end{aligned}
$$

The evaluation of the ring function involves the integral of $B[k], A[k]$, and hence involves the use of interpolators. For simplicity, we display the results of the computation using two different interpolators:

- closest grid: the off-grid quantity is approximated by its value on the closest momentum grid.

- 4-point difference: the off-grid quantity is obtained by Taylor expansion around the closest grid up to second order, the derivatives are obtained by 4-point difference formula.

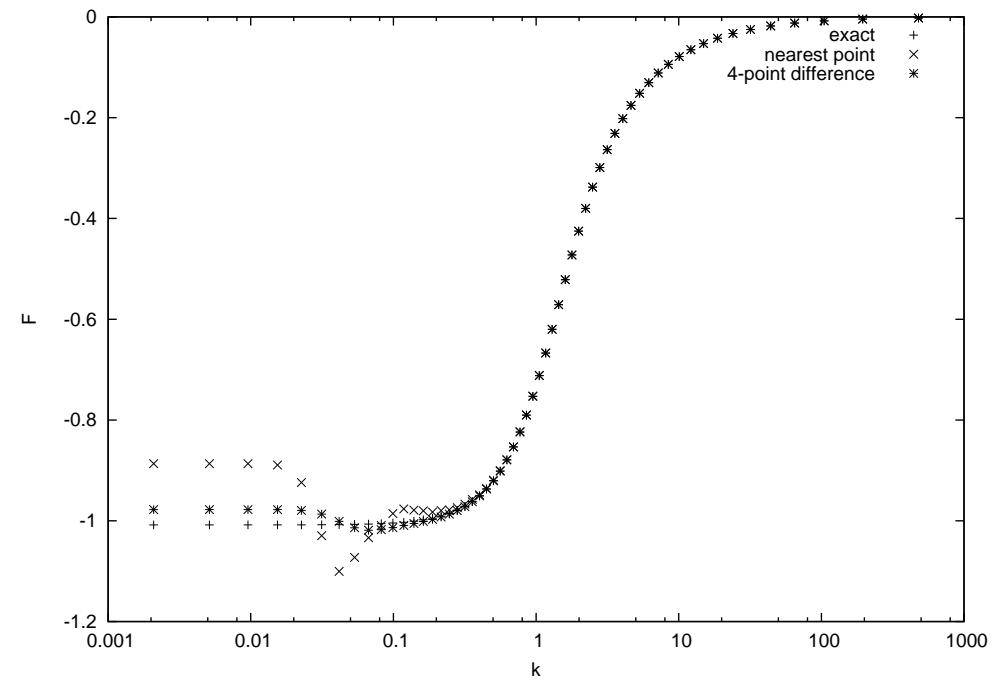

Figure 25: The computation of $F$ for modeled $B, A$ under various interpolating schemes.

It is apparent that the utilization of an improved interpolator scheme leads to a more reliable result in the computation. 
4.6.5.2 NUMERICAL METHODS Various algorithms are employed to solve the coupled integral equations in the study, which can be understood in the generic form of $\vec{X}=\vec{F}[\vec{X}]$. The main strategies include:

- natural iteration:

$$
X^{(n+1)}=F\left[X^{(n)}\right]
$$

- implicit iteration:

$$
X^{(n)}=F\left[X^{(n+1)}\right]
$$

- iteration with over-relaxation:

$$
X^{(n+1)}=(1-\omega) X^{(n)}+\omega F\left[X^{(n)}\right]
$$

- Newton-Raphson iteration:

$$
X^{(n+1)}=\left(1-\frac{\delta F}{\delta X}\left[X^{(n)}\right]\right)^{-1}\left(F\left[X^{(n)}\right]-X^{(n)}\right)
$$

- minimization:

$$
\min \left\|X^{(n)}-F\left[X^{(n)}\right]\right\|
$$


We find that natural iteration works well for all truncations except for BC and CP. Minimization is always stable, but can be quite time-consuming. Unfortunately, it can easily converge to one of the many local minima instead of the targeted global minimum. Extra care is required to select a suitable initial guess for the case of interest.

Mixed methods can be developed to deal with the feedback mechanism within the equations. For example, one can solve the fermionic part of the gap equations with an assumed form of the polarization by means of minimization, the resultant fermions 2-point functions can then be used to update the polarization, iterating this procedure allows one to enjoy both the stability of the minimization method while taking the advantage of the faster computation of the gap equations (by not computing the ring grid by grid in the integral). Of course, such a method will suffer from more intense interpolating effects, and hence it is essential to check the consistency of a given algorithm by comparing the results with the other methods.

\subsection{CONCLUSIONS}

We have studied dynamical parity and chiral symmetry breaking in QED3 within 2-spinor formalism. Schwinger-Dyson equations with various truncations, including the Ward identity preserving $\mathrm{BC}$ and $\mathrm{CP}$ vertex. The Coleman-Hill theorem is employed to check the robustness of the truncations.

Parity violating solutions are found for $\eta \lesssim 0.4$, while no solution can be found for the case of maximal violation $(\eta=1)$. It is interesting to note that the possibility of any parityviolating solution is at odds with the traditional view [52], this point certainly deserves further investigation.

Large ratios of mass scales are dynamically generated as one varies $N_{f}$. Critical $N_{f}$ for chiral restoration are reasonably close to that obtained by Appelquist et al., which is given by $N_{\star}^{A}=\frac{64}{\pi^{2}}$.

Coleman-Hill theorem is used as a diagnostic for truncation accuracy. It verifies our intuition that truncations which obey the Ward identity should perform better than those 
that are not. 


\subsection{QED3 AT FINITE TEMPERATURE AND DENSITY}

In this chapter ${ }^{1}$, we extend our study of QED3 to finite temperature and density. This generalization presents several challenges:

First, with the full frequency and momentum dependent non-perturbative photon propagators, the usual tricks to evaluate the Matsubara sums is no longer applicable. We must resort to direct numerical summation which greatly complicates our numerical study.

Second, at finite temperature, new infrared divergences arise in the fermion self energy. In the usual 4-spinor formalism, the divergence originates from the absence of the magnetic mass, which in turn is inferred from the Ward identity. Nevertheless, we shall argue that physical observables should be infrared safe.

Third, one needs to face the problem of non-perturbative renormalization at finite temperature. In principle, finite temperature effects should induce no new UV divergences so that one can simply renormalize at zero temperature. We shall see that the numerical implementation of this is highly non-trivial.

Keeping these issues in mind, we attempt a full field-theoretical calculation for QED3 at finite temperature. Our goal is to map out the phase structure and study the chiral phase transition.

\footnotetext{
${ }^{1}$ The research in this chapter is published in "Endemic infrared divergences in QED3 at finite temperature" [53].
} 


\subsection{TENSOR STRUCTURES AND POLARIZATION SCALARS IN FINITE TEMPERATURE FORMALISM}

At finite temperature, both the polarization tensor and the photon propagator remain transverse (up to a gauge term), while the presence of a 3 -vector $\hat{n}$ describing the heat bath allows us to define:

$$
k_{\perp}=k-n \frac{k^{2}}{n \cdot k},
$$

one can easily verify that:

$$
\begin{aligned}
k \cdot k_{\perp} & =0 \\
k_{\perp}^{2} & =\frac{n^{2} k^{2}-(n \cdot k)^{2}}{(n \cdot k)^{2}} k^{2},
\end{aligned}
$$

from this $\hat{k_{\perp}}$, one can define a set of six tensors:

$$
\begin{aligned}
& P_{1}=\hat{k_{\perp}^{\mu}} \hat{k_{\perp}^{\nu}} \\
& P_{2}=g^{\mu \nu}-\hat{k^{\mu}} \hat{k^{\nu}}-\hat{k_{\perp}^{\mu}} \hat{k_{\perp}^{\nu}} \\
& P_{3}=i \epsilon^{\mu \nu \rho} \hat{k_{\rho}} \\
& P_{4}=\hat{k_{\perp}^{\mu}} i \epsilon^{\nu \alpha \beta} \hat{k_{\alpha}} \hat{k_{\perp \beta}} \\
& P_{5}=i \epsilon^{\mu \alpha \beta} \hat{k_{\alpha}} \hat{k_{\perp \beta}} \hat{k_{\perp}^{\nu}} \\
& P_{6}=\epsilon^{\mu \alpha \beta} \epsilon^{\nu \alpha^{\prime} \beta^{\prime}} \hat{k_{\alpha}} \hat{k_{\perp \beta}} \hat{k_{\alpha^{\prime}}} \hat{k_{\perp \beta^{\prime}}} .
\end{aligned}
$$

This set of tensors form a closed space, and it is easily verified that

$$
k_{\mu} P_{I}^{\mu \nu}=0,
$$

for all six of them. It is important to note that: 


$$
\begin{aligned}
P_{1}+P_{2} & =g^{\mu \nu}-\hat{k^{\mu}} \hat{k^{\nu}} \\
P_{4} & =P_{1} P_{3} \\
P_{5} & =-P_{3} P_{1} \\
P_{6} & =-P_{5} P_{4} .
\end{aligned}
$$

With these definitions, it is straight forward to work out the following multiplication relations among the basis tensors:

Table 1: Multiplication relations for the set of tensors. The six transverse tensors form a closed space.

\begin{tabular}{|c|cccccc|}
\hline$\cdot$ & $P_{1}$ & $P_{2}$ & $P_{3}$ & $P_{4}$ & $P_{5}$ & $P_{6}$ \\
\hline$P_{1}$ & $P_{1}$ & 0 & $P_{4}$ & $P_{4}$ & 0 & 0 \\
$P_{2}$ & 0 & $P_{2}$ & $P_{3}-P_{4}$ & 0 & $P_{5}$ & $P_{6}$ \\
$P_{3}$ & $-P_{5}$ & $P_{3}+P_{5}$ & $P_{1}+P_{2}$ & $P_{6}$ & $-P_{1}$ & $P_{4}$ \\
$P_{4}=P_{1} P_{3}$ & 0 & $P_{4}$ & $P_{1}$ & 0 & $-P_{1}$ & $P_{4}$ \\
$P_{5}=-P_{3} P_{1}$ & $P_{5}$ & 0 & $-P_{6}$ & $-P_{6}$ & 0 & 0 \\
$P_{6}=-P_{5} P_{4}$ & 0 & $P_{6}$ & $-P_{5}$ & 0 & $P_{5}$ & $P_{6}$ \\
\hline
\end{tabular}

A note in using the table:

To compute $P_{I} \cdot P_{J}$ read from left column for $P_{I}$ and the top row for $P_{J}$, for example, from the table

$$
P_{5} \cdot P_{4}=-P_{6}
$$

while

$$
P_{4} \cdot P_{5}=-P_{1}
$$




\subsection{THE PHOTON PROPAGATOR}

Let's start with the bare photon propagator. The gap equation reads:

$$
D_{\mu \nu}^{(0)}{ }^{-1}=-k^{2}\left[g_{\mu \nu}-\left(1-\xi^{-1}\right) \frac{k_{\mu} k_{\nu}}{k^{2}}\right]-i \mu_{C S} \epsilon_{\mu \nu \sigma} k^{\sigma} .
$$

Inverting gives:

$$
D_{\mu \nu}^{(0)}=\frac{-1}{k^{2}-\mu_{C S}^{2}}\left[g_{\mu \nu}-\frac{k_{\mu} k_{\nu}}{k^{2}}-i \mu_{C S} \epsilon_{\mu \nu \sigma} k^{\sigma}\right]+\xi \frac{k_{\mu} k_{\nu}}{k^{2}} \frac{(-1)}{k^{2}} .
$$

For the full photon propagator, we allow all transverse structures to arise in the vacuum polarization. The gap equation reads:

$$
D_{\mu \nu}^{-1}=D_{\mu \nu}^{(0)}{ }^{-1}+i \Pi_{\mu \nu}
$$

We define:

$$
i \Pi_{\mu \nu}=\Pi_{j} P_{j}
$$

with $j=1,2,3, \ldots, 6$, hence

$$
\begin{aligned}
D_{\mu \nu}^{-1} & =-k^{2}\left(P_{1}+P_{2}\right)+\mu k P_{3}+-\xi^{-1} k^{2} \hat{k} \hat{k}+\Pi_{1} P_{1}+\Pi_{2} P_{2}+\Pi_{3} P_{3}+\Pi_{4} P_{4}+\Pi_{5} P_{5}+\Pi_{6} P_{6} \\
& =\left(-k^{2}+\Pi_{1}\right) P_{1}+\left(-k^{2}+\Pi_{2}\right) P_{2}+\left(\mu k+\Pi_{3}\right) P_{3}+-\xi^{-1} k^{2} \hat{k} \hat{k}+\Pi_{4} P_{4}+\Pi_{5} P_{5}+\Pi_{6} P_{6} .
\end{aligned}
$$

At this stage, it is already obvious that one can define

$$
\tilde{\Pi}_{3}=\Pi_{3}+\mu_{C S} k
$$

which will be seen as the dynamical and bare contribution of the photon mass, respectively. 
Hence our photonic gap equation reads:

$$
D_{\mu \nu}{ }^{-1}=\left(-k^{2}+\Pi_{1}\right) P_{1}+\left(-k^{2}+\Pi_{2}\right) P_{2}+\left(\tilde{\Pi}_{3}\right) P_{3}+-\xi^{-1} k^{2} \hat{k} \hat{k}+\Pi_{4} P_{4}+\Pi_{5} P_{5}+\Pi_{6} P_{6}
$$

Our goal is to compute the form of full photon propagator. To do that, we expand

$$
D=D_{j} P_{j}+C \hat{k} \hat{k}
$$

with $j=1,2,3, \ldots, 6$ to satisfy

$$
\left(D D^{-1}\right)^{\mu \nu}=g^{\mu \nu}=\left(P_{1}+P_{2}+\hat{k} \hat{k}\right)^{\mu \nu} .
$$

With explicit calculation, one reaches the following set of 6 equations:

$$
\begin{array}{r}
\left(-k^{2}+\Pi_{1}\right) D_{1}+\left(\tilde{\Pi}_{3}-\Pi_{5}\right) D_{3}+\left(\tilde{\Pi}_{3}-\Pi_{5}\right) D_{4}=1 \\
\left(-k^{2}+\Pi_{2}\right) D_{2}+\left(\tilde{\Pi}_{3}\right) D_{3}=1 \\
\left(\tilde{\Pi}_{3}\right) D_{2}+\left(-k^{2}+\Pi_{2}\right) D_{3}=0 \\
\left(\tilde{\Pi}_{3}+\Pi_{4}\right) D_{1}-\left(\tilde{\Pi}_{3}\right) D_{2}+\Pi_{6} D_{3}+\left(-k^{2}+\Pi_{2}+\Pi_{6}\right) D_{4}=0 \\
\Pi_{5} D_{2}+\left(\Pi_{2}-\Pi_{1}\right) D_{3}+\left(-k^{2}+\Pi_{1}\right) D_{5}-\left(\tilde{\Pi}_{3}-\Pi_{5}\right) D_{6}=0 \\
\Pi_{6} D_{2}+\left(\Pi_{4}\right) D_{3}-\left(\tilde{\Pi}_{3}+\Pi_{4}\right) D_{5}+\left(-k^{2}+\Pi_{2}+\Pi_{6}\right) D_{6}=0 .
\end{array}
$$

The gauge term is trivially solved:

$$
C=\frac{-\xi}{k^{2}}
$$

Another set of conventions that is commonly used in the literature reads: 


$$
\begin{aligned}
P_{1} & =P_{L} \\
\Pi_{1} & =k^{2} \Pi_{L} \\
P_{2} & =P_{\perp} \\
\Pi_{2} & =k^{2} \Pi_{\perp} .
\end{aligned}
$$

Also, to connect our previous discussion at zero temperature, we note that

$$
\begin{aligned}
F & \longrightarrow-\Pi_{1}=-\Pi_{2} \\
k \mathcal{F} & \longrightarrow \Pi_{3}
\end{aligned}
$$

In the current notation, all the П's have the dimension of $k^{2}$.

We employ the following strategy to solve the above system of equations:

Use the second and the third equation to solve for $D_{2}, D_{3}$, then the first and fourth for $D_{1}, D_{4}$, and finally fifth and sixth to reach for $D_{5}, D_{6}$.

The final results are: 


$$
\begin{aligned}
D_{1}= & \frac{-1}{k^{2}-\Pi_{1}-\frac{\left(\tilde{\Pi}_{3}+\Pi_{4}\right)\left(\tilde{\Pi}_{3}-\Pi_{5}\right)}{k^{2}-\Pi_{2}-\Pi_{6}}} \\
D_{2}= & \frac{-1}{k^{2}-\Pi_{2}-\frac{\tilde{\Pi}_{3}{ }^{2}}{k^{2}-\Pi_{2}}} \\
D_{3}= & \frac{\tilde{\Pi}_{3}}{k^{2}-\Pi_{2}} D_{2} \\
= & \frac{\tilde{\Pi}_{3}}{k^{2}-\Pi_{2}} \frac{-1}{k^{2}-\Pi_{2}-\frac{\tilde{\Pi}_{3}^{2}}{k^{2}-\Pi_{2}}} \\
D_{4}= & \frac{\tilde{\Pi}_{3}+\Pi_{4}}{k^{2}-\Pi_{2}-\Pi_{6}} D_{1}-\frac{\tilde{\Pi}_{3}}{k^{2}-\Pi_{2}} D_{2} \\
D_{5}= & \frac{-D_{1} D_{2}}{k^{2}-\Pi_{2}-\Pi_{6}}\left[\left(k^{2}-\Pi_{2}\right) \Pi_{5}+\tilde{\Pi}_{3}\left(\Pi_{2}-\Pi_{1}-\Pi_{6}\right)-\right. \\
& \left.\frac{\tilde{\Pi}_{3}}{k^{2}-\Pi_{2}}\left(\Pi_{6}\left(\Pi_{2}-\Pi_{1}\right)+\Pi_{4}\left(\tilde{\Pi}_{3}-\Pi_{5}\right)\right)\right] \\
D_{6}= & \frac{\Pi_{6} D_{2}+\Pi_{4} D_{3}-\left(\tilde{\Pi}_{3}+\Pi_{4}\right) D_{5}}{k^{2}-\Pi_{2}-\Pi_{6}} \\
C= & \frac{-\xi}{k^{2}} .
\end{aligned}
$$

The results presented are completely general, further simplification is possible if one works in a particular representation or imposes further assumptions.

\subsubsection{QED3 IN 4-SPINOR REPRESENTATION}

Within the 4 -spinor representation, $\Pi_{j}(j=3,4,5,6)$ are not dynamically generated, hence we have

$$
\tilde{\Pi}_{3}=\mu_{C S} k
$$

and the above $D_{j}$ 's reduce to: 


$$
\begin{aligned}
D_{1} & =\frac{-1}{k^{2}-\Pi_{1}-\frac{\left(\tilde{\Pi}_{3}\right)^{2}}{k^{2}-\Pi_{2}}} \\
D_{2} & =\frac{-1}{k^{2}-\Pi_{2}-\frac{\left(\tilde{\Pi}_{3}\right)^{2}}{k^{2}-\Pi_{2}}} \\
D_{3} & =\frac{\tilde{\Pi}_{3}}{k^{2}-\Pi_{2}} D_{2} \\
D_{4} & =\frac{\tilde{\Pi}_{3}}{k^{2}-\Pi_{2}}\left[D_{1}-D_{2}\right] \\
D_{5} & =-D_{4} \\
D_{6} & =\frac{-\tilde{\Pi}_{3}}{k^{2}-\Pi_{2}} D_{5} \\
C & =\frac{-\xi}{k^{2}} .
\end{aligned}
$$

\subsubsection{QED3 IN 2-SPINOR REPRESENTATION}

Using the 2-spinor representation, $\Pi_{j}(j=3,4,5,6)$ are dynamically generated. In fact, at one loop, $\Pi_{3}$ is dynamically generated, it corresponds to a topological photon mass term that breaks parity dynamically.

Whether or not $\Pi_{3}$ vanishes depends crucially on the structure of the vacuum: if parity is broken, it is non-zero; on the other hand, there exists systems of parity respecting solutions which make $\Pi_{3}=0$. This will be discussed below.

In the absence of $\tilde{\Pi}_{3}$, the above $D_{j}$ 's reduce to: 


$$
\begin{aligned}
D_{1} & =\frac{-1}{k^{2}-\Pi_{1}+\frac{\Pi_{4} \Pi_{5}}{k^{2}-\Pi_{2}-\Pi_{6}}} \\
D_{2} & =\frac{-1}{k^{2}-\Pi_{2}} \\
D_{3} & =0 \\
D_{4} & =\frac{\Pi_{4}}{k^{2}-\Pi_{2}-\Pi_{6}} D_{1} \\
D_{5} & =\frac{\Pi_{5}}{k^{2}-\Pi_{2}-\Pi_{6}} D_{1} \\
D_{6} & =\left[\frac{\Pi_{6}}{\Pi_{5}} \frac{k^{2}-\Pi_{1}}{k^{2}-\Pi_{2}}-\frac{\Pi_{4}}{k^{2}-\Pi_{2}}\right] D_{5} \\
C & =\frac{-\xi}{k^{2}}
\end{aligned}
$$

\subsection{ENDEMIC INFRARED DIVERGENCE IN QED3}

We shall turn to the study of the two especially important form factors for the polarization tensor: $\Pi_{1}$ and $\Pi_{2}$.

$\Pi_{1}$ is responsible for the electric screening, we shall see the matter part of $\Pi_{1}[0,0]$, defined as the electric mass square $m_{\mathrm{el}}^{2}$, is non-zero for QED3, while the corresponding magnetic mass $\Pi_{2}[0,0]=m_{m a g}^{2}$, is zero. This is a general non-perturbative statement derived from the Ward identities. The absence of the magnetic mass is the origin of the problem of infrared divergence for QED3 at finite temperature.

This problem is absent in the zero temperature version of the theory: the finite temperature theory is more divergent at zero Matsubara frequency. Previous attempts to address this issue include the explicit inclusion of an infrared cutoff [54], or assuming that higher order corrections would remove the divergence [55]. Many authors even try to evade the problem by neglecting all the spatial components of the photon propagators $[56,57,58]$, i.e.

$$
D_{\mu \nu}[\omega, \vec{k}] \longrightarrow D_{00}[\omega, \vec{k}]
$$


We shall argue that the problem of infrared divergence is endemic to QED3 at finite temperature. However, observables are finite and the theory is expected to be well-defined. The crucial observation is that the gauge term has the same form of infrared divergence, i.e. $\int d^{2} k 1 / k^{2}$. Hence the infrared divergence can be "gauged away", in other words, physical observables should be independent of this infrared divergence.

There is another rescue for the infrared divergence in QED3 in the 2-spinor formalism. There, $\Pi_{3}$ is dynamically generated, and hence may provide a natural infrared regulator for the various $D_{j}$ 's. However one has to be careful since the system of parity-breaking solution seems to be unstable and difficult to obtain. The case requires separate consideration.

\subsubsection{PERTURBATIVE STUDY OF THE POLARIZATION TENSOR}

We shall turn to explicit calculations of $\Pi_{1}, \Pi_{2}$ and $\Pi_{3}$ in perturbation theory. Recall that we define:

$$
i \Pi^{\mu \nu}=\Pi_{j} P_{j}^{\mu \nu}
$$

At one loop, only $\Pi_{1}, \Pi_{2}$ and $\Pi_{3}$ are generated. (To be more specific, for QED3 in 4-spinor, only $\Pi_{1}$ and $\Pi_{2}$, while in 2-spinor version, $\Pi_{3}$ is also dynamically generated, the use of BallChu vertex does not help to generate $\Pi_{4,5,6}$, but it includes their contribution non-trivially.)

For $P_{1}, P_{2}$ and $P_{3}$, their traces do not mix, hence it's easy to extract $\Pi_{1,2,3}$ from the $\Pi_{\mu \nu}$ (note that if $\Pi_{6}$ is generated, one needs to be more careful, for example $\operatorname{Tr}\left[P_{2} P_{6}\right]=\operatorname{Tr}\left[P_{6}\right]=$ 1 hence $\Pi_{2} \neq \operatorname{Tr}\left[P_{2} i \Pi\right]$.)

With the condition above, we obtain: (explicit calculation shows the best place to start is from $P_{2}$ )

$$
\begin{aligned}
& \Pi_{2}=\operatorname{Tr}\left[P_{2} i \Pi\right] \\
& \Pi_{1}=\operatorname{Tr}\left[P_{1} i \Pi\right]=g_{\mu \nu} i \Pi^{\mu \nu}-\Pi_{2} \\
& \Pi_{3}=\frac{1}{2} \operatorname{Tr}\left[P_{3} i \Pi\right]=\frac{1}{2} \epsilon_{\mu \nu \rho} \hat{k}^{\rho} \Pi^{\mu \nu} .
\end{aligned}
$$


Note that the order of $\mu \nu$ is altered to absorb the minus sign from the product of two $i$ 's to

extract $\Pi_{3}$ Also, as we will see, $\mathcal{F}=\frac{\Pi_{3}}{k}$ would be a more stable quantity to study. If we pick $n=[1,0,0]$, and perform the calculation at one loop, we have

$$
\begin{aligned}
& \Pi_{1}=-g^{2} \frac{1}{\beta} \sum_{\omega_{n}^{\prime}} \int \frac{d^{2} l}{(2 \pi)^{2}} \frac{1}{L_{1}^{2}-B_{1}^{2}} \frac{1}{L_{2}^{2}-B_{2}^{2}} 8\left[A_{1} A_{2}\left[\overrightarrow{l_{1}} \cdot \overrightarrow{l_{2}}-\frac{\left(\overrightarrow{l_{1}} \cdot \vec{k}\right)\left(\overrightarrow{l_{2}} \cdot \vec{k}\right)}{\vec{k}^{2}}\right]+B_{1} B_{2}\right] \\
& \Pi_{2}=g^{2} \frac{1}{\beta} \sum_{\omega_{n}^{\prime}} \int \frac{d^{2} l}{(2 \pi)^{2}} \frac{1}{L_{1}^{2}-B_{1}^{2}} \frac{1}{L_{2}^{2}-B_{2}^{2}} 4\left[L_{1}^{0} L_{2}^{0}+A_{1} A_{2}\left[\overrightarrow{l_{1}} \cdot \overrightarrow{l_{2}}-2 \frac{\left(\overrightarrow{l_{1}} \cdot \vec{k}\right)\left(\overrightarrow{l_{2}} \cdot \vec{k}\right)}{\vec{k}^{2}}\right]-B_{1} B_{2}\right] \\
& \frac{\Pi_{3}}{k}=\frac{-g^{2}}{2 k^{2}} \frac{1}{\beta} \sum_{\omega_{n}^{\prime}} \int \frac{d^{2} l}{(2 \pi)^{2}} \frac{1}{L_{1}^{2}-B_{1}^{2}} \frac{1}{L_{2}^{2}-B_{2}^{2}} 4\left[B_{2} L_{1} \cdot k-B_{1} L_{2} \cdot k\right],
\end{aligned}
$$

the perturbative expression can be obtained when we set

$$
\begin{gathered}
B=M \\
A=1 .
\end{gathered}
$$

To obtain the electric and magnetic screening mass, we shall take the limit of $k_{3}=0$ and $|\vec{k}| \rightarrow 0$ of $\Pi_{1}$ and $\Pi_{2}$ respectively. The explicit expression becomes:

$$
\begin{array}{r}
\Pi_{1}[0,0]=-g^{2} \frac{1}{\beta} \sum_{\omega_{n}^{\prime}} \int \frac{d^{2} l}{(2 \pi)^{2}} \frac{1}{\left[\omega_{n}^{\prime 2}+\epsilon_{l}^{2}\right]^{2}} 8\left[l^{2}\left(1-\cos ^{2}[\phi]\right)+M^{2}\right] \\
\left.\Pi_{2}[0,0]=g^{2} \frac{1}{\beta} \sum_{\omega_{n}^{\prime}} \int \frac{d^{2} l}{(2 \pi)^{2}} \frac{1}{\left[\omega_{n}^{\prime 2}+\epsilon_{l}^{2}\right]^{2}} 4\left[-\left[\omega_{n}^{\prime 2}+\epsilon_{l}^{2}\right]\right]+2 l^{2}\left(1-\cos ^{2}[\phi]\right)\right] .
\end{array}
$$

Also, we obtain the topological photon mass by taking the above limit for $\mathcal{F}=\frac{\Pi_{3}}{k}$

$$
\mathcal{F}[0,0]=-\frac{g^{2}}{2} \frac{1}{\beta} \sum_{\omega_{n}^{\prime}} \int \frac{d^{2} l}{(2 \pi)^{2}} \frac{1}{\left[\omega_{n}^{\prime 2}+\epsilon_{l}^{2}\right]^{2}} 4[M]
$$

or 


$$
\begin{aligned}
& \Pi_{1}[0,0]=-\alpha \frac{1}{\beta} \sum_{\omega_{n}^{\prime}} \int d l(l) 8 \frac{1}{\left[\omega_{n}^{\prime 2}+\epsilon_{l}^{2}\right]^{2}}\left[\epsilon_{l}^{2}+M^{2}\right] \\
& \Pi_{2}[0,0]=\alpha \frac{1}{\beta} \sum_{\omega_{n}^{\prime}} \int d l(l) 8\left[\frac{-1}{\left[\omega_{n}^{\prime 2}+\epsilon_{l}^{2}\right]}+\frac{\epsilon_{l}^{2}-M^{2}}{\left[\omega_{n}^{\prime 2}+\epsilon_{l}^{2}\right]^{2}}\right] \\
& \mathcal{F}[0,0]=-\alpha \frac{1}{\beta} \sum_{\omega_{n}^{\prime}} \int d l(l) 4 \frac{1}{\left[\omega_{n}^{\prime 2}+\epsilon_{l}^{2}\right]^{2}}[M] .
\end{aligned}
$$

We can compute the Matsubara sum analytically using the following formula:

$$
\frac{1}{\beta} \sum_{\omega_{n}^{\prime}} \frac{1}{\omega_{n}^{\prime 2}+\epsilon^{2}}=\frac{1}{2 \epsilon}[1-2 n]=\frac{1}{2 \epsilon} \tanh \left[\frac{1}{2} \beta \epsilon\right]
$$

and

$$
\frac{1}{\beta} \sum_{\omega_{n}^{\prime}} \frac{1}{\left[\omega_{n}^{\prime 2}+\epsilon^{2}\right]^{2}}=\frac{\tanh \left[\frac{1}{2} \beta \epsilon\right]-\frac{1}{2} \beta \epsilon\left[1-\tanh ^{2}\left[\frac{1}{2} \beta \epsilon\right]\right.}{4 \epsilon^{3}} .
$$

(generated easily from the first one by differentiating with respect to $\epsilon$ )

The matter piece of the $\Pi_{j}$ 's are obtained by subtracting the corresponding $T \rightarrow 0$ limit of the sum, technically, it amounts to replacing the Matsubara sum back to the integral over euclidean $k^{3}$ :

$$
\frac{1}{\beta} \sum_{\omega_{n}^{\prime}} \rightarrow \int \frac{d k^{3}}{2 \pi}
$$

with $k^{3}$ replacing $\omega_{n}^{\prime}$

Finally we reach:

$$
\Pi_{1}^{\mathrm{mat}}[0,0]=-\alpha T\left[\int_{x_{0}}^{\infty} d x \frac{1}{x^{2}}\left(x^{2}+x_{0}^{2}\right)\left[2\left(\tanh \left[\frac{1}{2} x\right]-1\right)-x\left[1-\tanh ^{2}\left[\frac{1}{2} x\right]\right]\right]\right.
$$

with 


$$
\begin{gathered}
x=\beta \epsilon \\
x_{0}=\beta M
\end{gathered}
$$

which can be computed analytically:

$$
m_{\mathrm{el}}^{2}=\Pi_{1}^{\mathrm{mat}}[0,0]=8 \alpha T\left[\frac{-x_{0} e^{x_{0}}}{1+e^{x_{0}}}+\ln \left[e^{x_{0}}+1\right]\right]
$$

with $x_{0}=\beta M$

Note that at $M \rightarrow 0$, we obtain the famous result:

$$
m_{\mathrm{el}}^{2}=8 \ln 2 \alpha T
$$

Hence, there is a well defined electric mass in our system, it regulates the infrared divergence for $P_{1}$ piece. (the longitudinal piece)

Now we perform the same calculation for the transverse piece $\Pi_{2}$ :

$$
\Pi_{2}^{\text {mat }}[0,0]=-\alpha T\left[\int_{x_{0}}^{\infty} d x\left[2 \frac{x^{2}+x_{0}^{2}}{x^{2}}\left(\tanh \left[\frac{1}{2} x\right]-1\right)+\frac{x^{2}-x_{0}^{2}}{x}\left[1-\tanh ^{2}\left[\frac{1}{2} x\right]\right]\right]\right.
$$

with

$$
\begin{gathered}
x=\beta \epsilon \\
x_{0}=\beta M
\end{gathered}
$$

which is analytically zero!

With gauge symmetry, it can be shown that the magnetic mass vanishes for all orders.

Hence, the transverse piece still has the problem of infrared divergence in the absence of the topological photon mass, i.e $\tilde{\Pi}_{3}=0$.

Lastly, and crucially, we perform the same calculation for $\mathcal{F}=\frac{\Pi_{3}}{k}$ : 


$$
\mathcal{F}^{\text {mat }}[0,0]=-\alpha x_{0} \int_{x_{0}}^{\infty} d x \frac{1}{x^{2}}\left[\left(\tanh \left[\frac{1}{2} x\right]-1\right)-\frac{1}{2} x\left[1-\tanh ^{2}\left[\frac{1}{2} x\right]\right]\right]
$$

with

$$
\begin{gathered}
x=\beta \epsilon \\
x_{0}=\beta M,
\end{gathered}
$$

which, analytically, is given by

$$
\mathcal{F}^{\text {mat }}[0,0]=-\alpha\left[\tanh \left[\frac{x_{0}}{2}\right]-1\right]
$$

Note that for $\mathcal{F}$, there is actually a vacuum piece given by:

$$
\mathcal{F}^{\mathrm{vac}}[0,0]=-\alpha \frac{M}{|M|}
$$

combining the two results gives

$$
\mathcal{F}^{\text {full }}[0,0]=-\alpha\left[\tanh \left[\frac{M}{2 T}\right]\right]
$$

which is ill-defined at $M \rightarrow 0$ and $T \rightarrow 0$, but is otherwise an valid expression. 


\subsubsection{ABSENCE OF MAGNETIC MASS IN QED3}

It is useful to review the arguments used in showing that the magnetic mass vanishes for all orders $[59,60]$.

Consider the polarization tensor:

$$
\Pi_{\mu \nu}(n, q) \sim e^{2} T \sum_{\nu} \int d^{2} \ell \operatorname{tr}\left[\gamma_{\mu} S(\ell) \Gamma_{\nu}(q+\ell, \ell) S(q+\ell)\right] .
$$

From Ward identity, we have

$$
\Gamma_{\nu}(\ell, \ell)=\frac{\partial S^{-1}(\ell)}{\partial \ell_{\nu}}
$$

Noticing that:

$$
\frac{\partial S^{-1}}{\partial \ell_{\nu}}=-S^{-1} \frac{\partial S}{\partial \ell_{\nu}} S^{-1}
$$

we finally reach:

$$
\Pi_{\mu \nu}(0, q \rightarrow 0) \sim-e^{2} T \sum_{\nu} \int d^{2} \ell \operatorname{tr}\left[\gamma_{\mu} \frac{\partial S}{\partial q_{\nu}}\right]
$$

Notice that the spatial part $(i, j)$ of the tensor vanishes due to complete derivative, which is exactly the contribution to magnetic mass. 


\subsubsection{ENDEMIC INFRARED DIVERGENCE}

We are now in position to understand the existence of the infrared divergence problem in the gap equations of QED3.

Consider

$$
S_{p}^{-1}=\not p-\Sigma
$$

From the gap equation, we obtain:

$$
-\Sigma=g^{2} \frac{1}{\beta} \sum \int \frac{d^{2} l}{(2 \pi)^{2}} \Gamma^{\mu}[l, p] S_{l} \gamma^{\nu} D_{\mu \nu}[p-l] .
$$

We define $\eta=p-l$, the relevant infrared divergence comes from $\eta \rightarrow 0$ limit in the integral, i.e

$$
\begin{aligned}
-\Sigma_{d i v} & =g^{2} \frac{1}{\beta} \sum \int_{d i v} \frac{d^{2} \vec{\eta}}{(2 \pi)^{2}} \Gamma^{\mu}[p, p] S_{p} \gamma^{\nu} D_{\mu \nu}[\eta] \\
& =g^{2} \frac{1}{\beta} \Gamma^{\mu}[p, p] S_{p} \gamma^{\nu} \int_{d i v} \frac{d^{2} \vec{\eta}}{(2 \pi)^{2}} D_{\mu \nu}[\eta],
\end{aligned}
$$

where we focus on $\eta^{0}=0$ part of the sum for it is the source of infrared divergence.

It is now clear that the key to understanding the infrared divergence is in the study of the integral

$$
\int_{d i v} \frac{d^{2} \vec{\eta}}{(2 \pi)^{2}} D_{\mu \nu}[\eta]
$$

Recall $D[k]=D_{j} P_{j}$ and the definition of $k_{\perp}$ as

$$
k_{\perp}=k-\frac{n k^{2}}{n \cdot k}
$$

then 


$$
\begin{aligned}
k_{\perp}^{2} & =\frac{n^{2} k^{2}-(n \cdot k)^{2}}{(n \cdot k)^{2}} k^{2} \\
k_{\perp}^{-2} & =\frac{1}{k^{2}} \frac{(n \cdot k)^{2}}{n^{2} k^{2}-(n \cdot k)^{2}} .
\end{aligned}
$$

We pick $n=[1,0,0]$ and study $k^{0}=0$ limit to study the IR divergence limit of the tensor, that is, we pick $n \cdot k=0$, we obtain the following: (the integral is integrating over $d^{2} \vec{k}$, remember $k^{0}=0$ with the current choice of $n$ )

$$
\begin{aligned}
& \int \hat{k} \hat{k}=\frac{1}{2}[g-n n] \\
& \int P_{1}=\int n n \\
& \int P_{2}=\int \frac{1}{2}[g-n n] \\
& \int P_{3}=0 \\
& \int P_{4}=0 \\
& \int P_{5}=0 \\
& \int P_{6}=\int \frac{1}{2}[g-n n],
\end{aligned}
$$

with these limits, one obtain

$$
\int_{d i v} \frac{d^{2} \vec{\eta}}{(2 \pi)^{2}} D_{\mu \nu}[\eta]=\int_{d i v} \frac{d^{2} \vec{\eta}}{(2 \pi)^{2}}\left[n n D_{1}+\frac{1}{2}[g-n n]\left[D_{2}+D_{6}+-\frac{\xi}{\eta^{2}}\right]\right]_{\mu \nu} .
$$

Hence, we have the expression:

$$
-\Sigma_{d i v}=g^{2} \frac{1}{\beta} \Gamma^{\mu}[p, p] S_{p} \gamma^{\nu} \int_{d i v} \frac{d^{2} \vec{\eta}}{(2 \pi)^{2}}\left[n n D_{1}+\frac{1}{2}[g-n n]\left[D_{2}+D_{6}+-\frac{\xi}{\eta^{2}}\right]\right]_{\mu \nu}
$$


As we have motivated, for those $D_{j}$ 's that are proportional to $\frac{1}{\eta^{2}}$ similar to the gauge term, we will have an infrared divergence.

We have already shown that $D_{1}$ is infrared safe, due to the existence of the electric mass, let's focus on $D_{2}$ and $D_{6}$.

Explicitly, for QED3 in 4-spinor without $\Pi_{3}$ :

$$
\begin{aligned}
& D_{1} \longrightarrow \frac{-1}{\eta^{2}-\Pi_{1}[\eta]} \\
& D_{2} \longrightarrow \frac{-1}{\eta^{2}-\Pi_{2}[\eta]} \\
& D_{6} \longrightarrow 0
\end{aligned}
$$

We have seen that $\Pi_{2}$ does not have the magnetic mass, hence, we can write

$$
\Pi_{2}[\eta] \rightarrow c_{2} \eta^{2}
$$

hence we have (note that $\eta^{2}=-\vec{\eta}^{2}$ )

$$
\int_{d i v} \frac{d^{2} \vec{\eta}}{(2 \pi)^{2}} D[\eta] \rightarrow \int_{d i v} \frac{d^{2} \vec{\eta}}{(2 \pi)^{2}} \frac{1}{2}[g-n n]\left[\frac{1}{1-c_{2}} \frac{1}{\vec{\eta}^{2}}+\frac{\xi}{\vec{\eta}^{2}}\right]
$$

Finally we get:

$$
-\Sigma_{d i v}=\alpha \frac{1}{\beta} \Gamma^{\mu}[p, p] S_{p} \gamma^{\nu}[g-n n]_{\mu \nu}\left[-\ln \left[\epsilon_{I R}\right]\right]\left[\frac{1}{1-c_{2}}+\xi\right]
$$

note that we regulate the integral and define $\int d l \frac{1}{l}=-\ln \left[\epsilon_{I R}\right]$.

A similar study in 2-spinor formalism, defining $\Pi_{6}[\eta] \rightarrow c_{6} \eta^{2}$, leads to

$$
-\Sigma_{d i v}=\alpha \frac{1}{\beta} \Gamma^{\mu}[p, p] S_{p} \gamma^{\nu}[g-n n]_{\mu \nu}\left[-\ln \left[\epsilon_{I R}\right]\right]\left[\frac{1}{1-c_{2}}+\frac{c_{6}}{\left(1-c_{2}\right)\left(1-c_{2}-c_{6}\right)}+\xi\right]
$$

The key observation to resolve the issue is to recognize that the gauge term itself has the same form of infrared divergence as the ones discussed above. Therefore, for physical quantities to be gauge independent, they must be independent of this infrared divergence. In principle, 
we can regulate the various infrared divergences freely and be confident that the infrared divergences can be "gauged away".

Alternatively, the existence of $\tilde{\Pi}_{3}$ and a parity breaking solution can also help to rescue all $D_{j}$ 's, leaving only the gauge term to be infrared divergent.

\subsection{SCHWINGER-DYSON EQUATIONS}

For simplicity, we shall confine our analysis of the gap equations in 4-spinor formalism, where parity is not dynamically broken. Moreover, we shall employ the rainbow ladder truncation in our study.

\subsubsection{THE GAP EQUATIONS}

5.4.1.1 FERMIONS Starting with the Ansätz:

$$
S^{-1}=A_{0}[p]\left(i \omega_{n}+\mu\right) \gamma^{0}-A[p] \vec{p} \cdot \vec{\gamma}-B[p]
$$

The gap equations read

$$
\begin{aligned}
& A_{0}[p]\left(i \omega_{n}+\mu\right) \gamma^{0}-A[p] \vec{p} \cdot \vec{\gamma}-B[p]=A_{0}[p]\left(i \omega_{n}+\mu\right) \gamma^{0}-\vec{p} \cdot \vec{\gamma}-m+ \\
& g^{2} \frac{1}{\beta} \sum_{\omega_{n}^{\prime}} \int \frac{d^{2} l}{(2 \pi)^{2}} \gamma^{\mu} \frac{1}{A_{0}[l]\left(i \omega_{n}^{\prime}+\mu\right) \gamma^{0}-A[l] \vec{l} \cdot \vec{\gamma}-B[l]} \gamma^{\nu} D_{\mu \nu}[p-l],
\end{aligned}
$$

we reach: 


$$
\begin{aligned}
B[p]= & m+g^{2} \frac{1}{\beta} \sum_{\omega_{n}^{\prime}} \int \frac{d^{2} l}{(2 \pi)^{2}} \frac{1}{A_{0}[l]^{2}\left(\omega_{n}^{\prime}-i \mu\right)^{2}+A[l]^{2} \vec{l}^{2}+B[l]^{2}}\left[B_{l} g_{\mu \nu} D^{\mu \nu}\right] \\
A[p]=1+ & g^{2} \frac{1}{\beta} \sum_{\omega_{n}^{\prime}} \int \frac{d^{2} l}{(2 \pi)^{2}} \frac{-1}{A_{0}[l]^{2}\left(\omega_{n}^{\prime}-i \mu\right)^{2}+A[l]^{2} \overrightarrow{l^{2}}+B[l]^{2}} \frac{1}{\vec{p}^{2}}\left[p^{i} L_{\beta}\left(D^{i \beta}+D^{\beta i}\right)\right. \\
& \left.\quad-p^{i} L^{i} g_{\mu \nu} D^{\mu \nu}\right] \\
A_{0}[p]=1+ & g^{2} \frac{1}{\beta} \sum_{\omega_{n}^{\prime}} \int \frac{d^{2} l}{(2 \pi)^{2}} \frac{-1}{A_{0}^{2}\left(\omega_{n}^{\prime}-i \mu\right)^{2}+A_{l}^{2} \vec{l}^{2}+B_{l}^{2}}\left[L_{\beta}\left(D^{0 \beta}+D^{\beta 0}\right)-L^{0} g_{\mu \nu} D^{\mu \nu}\right]
\end{aligned}
$$

where

$$
\begin{aligned}
L & :\left[A_{0}\left(i \omega_{n}^{\prime}+\mu\right), A_{l} \vec{l}\right] \\
p & :\left[i \omega_{n}, \vec{p}\right] \\
l & :\left[i \omega_{n}^{\prime}, \vec{l}\right] \\
D^{\mu \nu} & =D^{\mu \nu}[p-l]
\end{aligned}
$$

As have been discussed in the previous sections, the photon propagator takes the form

$$
\begin{aligned}
D_{\mu \nu}[k] & =\frac{1}{k^{2}+F} P_{L_{\mu \nu}}+\frac{1}{k^{2}+G} P_{T \mu \nu}-\frac{\xi}{k^{2}} \frac{k_{\mu} k_{\nu}}{k^{2}} \\
& =\frac{1}{k^{2}+F}-\left[g_{\mu \nu}-\frac{k_{\mu} k_{\nu}}{k^{2}}\right]+\frac{F-G}{\left(k^{2}+F\right)\left(k^{2}+G\right)} P_{T \mu \nu}-\frac{\xi}{k^{2}} \frac{k_{\mu} k_{\nu}}{k^{2}}
\end{aligned}
$$

with

$$
\begin{gathered}
P_{0 \nu}^{T}=P_{\nu 0}^{T}=0 \\
P_{i j}^{T}=-g_{i j}-\frac{k_{i} k_{j}}{\vec{k}^{2}} \\
P_{\mu \nu}^{T}+P_{\mu \nu}^{L}=-\left[g_{\mu \nu}-\frac{k_{\mu} k_{\nu}}{k^{2}}\right] .
\end{gathered}
$$

We shall go through the derivation of $F$ and $G$, the finite temperature polarization scalar functions, in the next section. 


\subsubsection{PHOTONS}

$$
D_{\mu \nu}^{-1}[k]=D_{\mu \nu}^{(0)^{-1}}[k]+i g^{2} \operatorname{Tr}\left[\gamma_{\mu} S \Gamma_{\nu} S\right]
$$

and under the ladder approximation, we have:

$$
D_{\mu \nu}^{-1}[k]=D_{\mu \nu}^{(0)^{-1}}[k]+i g^{2} \operatorname{Tr}\left[\gamma_{\mu} S \gamma_{\nu} S\right]
$$

it is useful to define $\Pi^{\mu \nu}[k]$ such that:

$$
D_{\mu \nu}{ }^{-1}[k]=D_{\mu \nu}^{(0)^{-1}}[k]+i \Pi_{\mu \nu}[k]
$$

with

$$
\Pi^{\mu \nu}[k]=i g^{2} \frac{1}{\beta} \sum_{\omega_{n}^{\prime}} \int \frac{d^{2} l}{(2 \pi)^{2}} \frac{1}{L_{1}^{2}-B_{1}^{2}} \frac{1}{L_{2}^{2}-B_{2}^{2}} \operatorname{Tr}\left[\gamma^{\mu}\left(L / 1+B_{1}\right) \gamma^{\nu}\left(L_{2}+B_{2}\right)\right]
$$

performing the trace calculation we reach:

$$
\Pi^{\mu \nu}[k]=i g^{2} \frac{1}{\beta} \sum_{\omega_{n}^{\prime}} \int \frac{d^{2} l}{(2 \pi)^{2}} \frac{1}{L_{1}^{2}-B_{1}^{2}} \frac{1}{L_{2}^{2}-B_{2}^{2}} 4\left[\left(L_{1}^{\mu} L_{2}^{\nu}+L_{1}^{\nu} L_{2}^{\mu}-g^{\mu \nu} L_{1} \cdot L_{2}\right)+g^{\mu \nu} B_{1} B_{2}\right]
$$

where

$$
\begin{aligned}
L_{1}^{\mu} & =\left[A_{0}\left(i \omega_{n}^{\prime}+\mu\right), A_{l} \overrightarrow{l_{1}}\right] \\
L_{2}^{\mu} & =\left[A_{0}\left(i \omega_{n}^{\prime}-i \nu_{n}+\mu\right), A_{2} \overrightarrow{l_{2}}\right] \\
k & =\left[i \nu_{n}, \vec{k}\right]
\end{aligned}
$$

and 


$$
\begin{aligned}
& \overrightarrow{l_{1}}=\vec{l} \\
& \overrightarrow{l_{2}}=\vec{l}-\vec{k}
\end{aligned}
$$

Now we focus on the various tensor properties of the photon propagator:

$$
D_{\mu \nu}^{(0)}[k]=\frac{-\left[g_{\mu \nu}-(1-\xi) \frac{k_{\mu} k_{\nu}}{k^{2}}\right]}{k^{2}}
$$

One can also work out the inverse bare photon propagator:

$$
D_{\mu \nu}^{(0)^{-1}}[k]=-k^{2}\left[g_{\mu \nu}-\frac{k_{\mu} k_{\nu}}{k^{2}}\right]-\frac{1}{\xi} k_{\mu} k_{\nu}
$$

such that

$$
D_{\mu \alpha}^{(0)^{-1}} D^{(0)^{\alpha \nu}}=g_{\mu}^{\nu}
$$

Now we define

$$
i \Pi^{\mu \nu}=F P_{L}^{\mu \nu}+G P_{T}^{\mu \nu}
$$

with

$$
P_{\mu \nu}^{T}+P_{\mu \nu}^{L}=-\left[g_{\mu \nu}-\frac{k_{\mu} k_{\nu}}{k^{2}}\right]
$$

$P_{\mu \nu}^{T}$ satisfies:

$$
P_{0 \nu}^{T}=P_{\nu 0}^{T}=0
$$




$$
P_{i j}^{T}=-g_{i j}-\frac{k_{i} k_{j}}{\vec{k}^{2}}
$$

One can check that

$$
\begin{gathered}
P_{T}^{\mu \alpha} P_{\alpha \nu}^{T}=-P_{T_{\nu}^{\mu}}^{\mu} \\
P_{L}^{\mu \alpha} P_{\alpha \nu}^{L}=-P_{L_{\nu}^{\mu}}^{\mu} \\
P_{L}^{\mu \alpha} P_{\alpha \nu}^{T}=0
\end{gathered}
$$

also observes that

$$
\begin{aligned}
& k_{\mu} P_{T}^{\mu \nu}=0 \\
& k_{\mu} P_{L}^{\mu \nu}=0
\end{aligned}
$$

yet another useful result is:

$$
\begin{aligned}
& P_{T_{\mu}^{\mu}}^{\mu}=-(d-2) \\
& P_{L_{\mu}^{\mu}}^{\mu}=-1
\end{aligned}
$$

Then, a direct substitution gives

$$
D_{\mu \nu}^{-1}[k]=\left(k^{2}+F\right) P_{\mu \nu}^{L}+\left(k^{2}+G\right) P_{\mu \nu}^{T}-\frac{1}{\xi} k_{\mu} k_{\nu}
$$

inverting gives: 


$$
\begin{aligned}
D_{\mu \nu}[k] & =\frac{1}{k^{2}+F} P_{L \mu \nu}+\frac{1}{k^{2}+G} P_{T \mu \nu}-\frac{\xi}{k^{2}} \frac{k_{\mu} k_{\nu}}{k^{2}} \\
& =\frac{1}{k^{2}+F}-\left[g_{\mu \nu}-\frac{k_{\mu} k_{\nu}}{k^{2}}\right]+\frac{F-G}{\left(k^{2}+F\right)\left(k^{2}+G\right)} P_{T \mu \nu}-\frac{\xi}{k^{2}} \frac{k_{\mu} k_{\nu}}{k^{2}}
\end{aligned}
$$

From the definition of $\Pi^{\mu \nu}$ :

$$
i \Pi^{\mu \nu}=F P_{L}^{\mu \nu}+G P_{T}^{\mu \nu}
$$

one can fish out $F$ and $G$ :

$$
G=\frac{1}{d-2}\left[\delta_{i j}-\frac{k_{i} k_{j}}{\vec{k}^{2}}\right] i \Pi^{i j}
$$

and

$$
F=-\left(g_{\mu \nu}-\frac{k_{\mu} k_{\nu}}{k^{2}}\right) i \Pi^{\mu \nu}-(d-2) G
$$

note that

$$
k_{\mu} \Pi^{\mu \nu}=0 .
$$

In QED3, we finally reach:

$$
\begin{gathered}
G=\left[\delta_{i j}-\frac{k_{i} k_{j}}{\vec{k}^{2}}\right] i \Pi^{i j} \\
F=-i g_{\mu \nu} \Pi^{\mu \nu}-G
\end{gathered}
$$

with the form of $\Pi^{\mu \nu}$ above, we have: 


$$
\begin{aligned}
G\left[\nu_{n} \vec{k}\right]= & -g^{2} \frac{1}{\beta} \sum_{\omega_{n}^{\prime}} \int \frac{d^{2} l}{(2 \pi)^{2}} \frac{1}{L_{1}^{2}-B_{1}^{2}} \frac{1}{L_{2}^{2}-B_{2}^{2}} 4\left[L_{1}^{0} L_{2}^{0}+A_{1} A_{2} \overrightarrow{l_{1}} \cdot \overrightarrow{l_{2}}-B_{1} B_{2}\right. \\
& \left.-\frac{2 A_{1} A_{2}\left(\overrightarrow{l_{1}} \cdot \vec{k}\right)\left(\overrightarrow{l_{2}} \cdot \vec{k}\right)}{\vec{k}^{2}}\right] \\
F\left[\nu_{n} \vec{k}\right]= & g^{2} \frac{1}{\beta} \sum_{\omega_{n}^{\prime}} \int \frac{d^{2} l}{(2 \pi)^{2}} \frac{1}{L_{1}^{2}-B_{1}^{2}} \frac{1}{L_{2}^{2}-B_{2}^{2}} 4\left[-L_{1} \cdot L_{2}+3 B_{1} B_{2}\right]-G_{\nu_{n} \vec{k}} \\
= & g^{2} \frac{1}{\beta} \sum_{\omega_{n}^{\prime}} \int \frac{d^{2} l}{(2 \pi)^{2}} \frac{1}{L_{1}^{2}-B_{1}^{2}} \frac{1}{L_{2}^{2}-B_{2}^{2}} 4(2)\left[A_{1} A_{2}\left(\overrightarrow{l_{1}} \cdot \overrightarrow{l_{2}}-\frac{\overrightarrow{l_{1}} \cdot \vec{k} \overrightarrow{l_{2}} \cdot \vec{k}}{\vec{k}^{2}}\right)+B_{1} B_{2}\right]
\end{aligned}
$$

with

$$
\begin{aligned}
L_{1}^{\mu} & =\left[A_{0}\left(i \omega_{n}^{\prime}+\mu\right), A_{l} \overrightarrow{l_{1}}\right] \\
L_{2}^{\mu} & =\left[A_{0}\left(i \omega_{n}^{\prime}-i \nu_{n}+\mu\right), A_{2} \overrightarrow{l_{2}}\right] \\
k & =\left[i \nu_{n}, \vec{k}\right]
\end{aligned}
$$

and

$$
\begin{aligned}
& \overrightarrow{l_{1}}=\vec{l} \\
& \overrightarrow{l_{2}}=\vec{l}-\vec{k}
\end{aligned}
$$

This is the ultimate form of the two polarization scalars at finite temperature of QED3. 


\subsubsection{A NOTE FOR ZERO TEMPERATURE AND DENSITY}

At zero temperature and density, one can show that

$$
\Pi^{\mu \nu} \propto-\left[g^{\mu \nu}-\frac{k^{\mu} k^{\nu}}{k^{2}}\right]
$$

and hence

$$
\begin{gathered}
F=G \\
i \Pi^{\mu \nu}=-\left[g^{\mu \nu}-\frac{k^{\mu} k^{\nu}}{k^{2}}\right] F
\end{gathered}
$$

or

$$
F=-\frac{1}{2}\left[g^{\mu \nu}-\eta \frac{k^{\mu} k^{\nu}}{k^{2}}\right] i \Pi_{\mu \nu} .
$$

As discussed in section 4.3.3.2, we can exploit the freedom of transversality and pick $\eta=3$, so that the expression is automatically finite and renormalized.

With the explicit expression of $\Pi_{\mu \nu}$, one can construct $F[k ; B, A]$

$$
\begin{aligned}
F= & -i \frac{1}{2}\left[g_{\mu \nu}-3 \frac{k_{\mu} k_{\nu}}{k^{2}}\right] g^{2} \int \frac{d^{3} l}{(2 \pi)^{3}} \frac{1}{A_{1}^{2} l_{1}^{2}-B_{1}^{2}} \frac{1}{A_{2}^{2} l_{2}^{2}-B_{2}^{2}} \operatorname{Tr}[I]\left[A _ { 1 } A _ { 2 } \left[l_{1}^{\mu} l_{2}^{\nu}+\right.\right. \\
& \left.\left.l_{2}^{\mu} l_{1}^{\nu}-g^{\mu \nu} l_{1} \cdot l_{2}\right]+g^{\mu \nu} B_{1} B_{2}\right]
\end{aligned}
$$

where

$$
\begin{aligned}
& l_{1}^{\mu}=l^{\mu} \\
& l_{2}^{\mu}=l^{\mu}-k^{\mu} .
\end{aligned}
$$

A very useful limit to study is the perturbative result: 
Setting:

$$
\begin{gathered}
B=M \\
A=1 .
\end{gathered}
$$

we would have: (Minkowski space result)

$$
F=G=-\frac{1}{2} \operatorname{Tr}[I] \frac{g^{2}}{4 \pi}\left(-2 k^{2}\right) \int_{0}^{1} d \alpha \alpha(1-\alpha) \frac{1}{\sqrt{M^{2}-\alpha(1-\alpha) k^{2}}}
$$

\subsection{ASPECTS OF ELECTRIC SCREENING}

In section 5.3, we have defined the electric screening mass $m_{\mathrm{el}}$ as the zero momentum limit of $\Pi_{1}$ and presented its perturbative limit. It turns out that the non-perturbative computation of this quantity is far from trivial.

\subsubsection{DEFINITION}

Recall that the electric screening mass is defined by:

$$
m_{\mathrm{el}}^{2}=\Pi_{1}^{\mathrm{mat}}[0,0]
$$

where the matter part of $\Pi_{1}$ is obtained by subtracting the corresponding zero-temperature piece away from the full expression.

In the non-perturbative case, $\Pi_{1}$ is a functional of the full fermion propagator (within the current truncation scheme). The notion of "subtracting the zero-temperature piece" leads to the following confusion:

$$
\Pi_{1}{ }^{R}\left[B, A, A_{0}\right](0,0) \stackrel{?}{=} \Pi_{1}\left[B, A, A_{0}\right](0,0)-\Pi_{1}^{T=0}\left[B, A, A_{0}\right](0,0) .
$$


Given the full fermion propagator, $\Pi_{1}\left[B, A, A_{0}\right]$ reads

$$
\Pi_{1}\left[\nu_{n} \vec{k}\right]=g^{2} \frac{1}{\beta} \sum_{\omega_{n}^{\prime}} \int \frac{d^{2} l}{(2 \pi)^{2}} \frac{1}{L_{1}^{2}-B_{1}^{2}} \frac{1}{L_{2}^{2}-B_{2}^{2}} 4(2)\left[A_{1} A_{2}\left(\overrightarrow{l_{1}} \cdot \overrightarrow{l_{2}}-\frac{\overrightarrow{l_{1}} \cdot \vec{k} \overrightarrow{l_{2}} \cdot \vec{k}}{\vec{k}^{2}}+B_{1} B_{2}\right]\right.
$$

with

$$
\begin{aligned}
L_{1}^{\mu} & =\left[A_{0}\left(i \omega_{n}^{\prime}+\mu\right), A_{l} \overrightarrow{l_{1}}\right] \\
L_{2}^{\mu} & =\left[A_{0}\left(i \omega_{n}^{\prime}-i \nu_{n}+\mu\right), \overrightarrow{A_{2}} \overrightarrow{l_{2}}\right] \\
k & =\left[i \nu_{n}, \vec{k}\right] .
\end{aligned}
$$

The tricky part is the subtraction of zero temperature piece. Naively, one would be tempted to form the following expression:

$$
\Pi_{1}^{T=0}\left[\nu_{n} \vec{k}\right]=g^{2} \int \frac{d l^{3}}{2 \pi} \int \frac{d^{2} l}{(2 \pi)^{2}} \frac{1}{L_{1}^{2}-B_{1}^{2}} \frac{1}{L_{2}^{2}-B_{2}^{2}} 4(2)\left[A_{1} A_{2}\left(\overrightarrow{l_{1}} \cdot \overrightarrow{l_{2}}-\frac{\overrightarrow{l_{1}} \cdot \vec{k} \overrightarrow{l_{2}} \cdot \vec{k}}{\vec{k}^{2}}+B_{1} B_{2}\right]\right.
$$

with

$$
\begin{aligned}
L_{1}^{\mu} & =\left[A_{0}\left(i l^{3}\right), A_{l} \overrightarrow{l_{1}}\right] \\
L_{2}^{\mu} & =\left[A_{0}\left(i l^{3}-i \nu_{n}\right), A_{2} \overrightarrow{l_{2}}\right] \\
k & =\left[i \nu_{n}, \vec{k}\right],
\end{aligned}
$$

that is, replace the above Matsubara sum back to an integral over Euclidean $l^{3}$.

Of course, the above expression is intuitively true in the perturbative limit. However, we observe the following two discrepancies in this definition of $\Pi^{T=0}$.

Firstly, the full fermion propagator is defined only on the discrete Matsubara frequency grids:

$$
\begin{array}{r}
B\left[\omega_{n}, \vec{l}\right], A\left[\omega_{n}, \vec{l}\right], A_{0}\left[\omega_{n}, \vec{l}\right] \\
\omega_{n}=\frac{(2 n+1) \pi}{\beta} .
\end{array}
$$


In order to compute the integral version of the expression, one has to perform an analytic continuation for the full fermionic propagator.

The need for analytic continuation introduces the second layer of complexity: what limit do we analytically continue to?

If we consider the functions that "connect" all points on the Matsubara frequency grids, a deeper inspection would indicate that such a continuation does not correspond to the solution at zero temperature, which violates what we set out to do, namely, renormalization at zero temperature.

The correct expression for our purpose is given by:

$$
\Pi_{1}^{R}\left[B, A, A_{0}\right](0,0)=\Pi_{1}\left[B, A, A_{0}\right](0,0)-\Pi_{1}^{T=0}\left[B^{T=0}, A^{T=0}, A_{0}^{T=0}=A^{T=0}\right](0,0)
$$

where $B^{T=0}, A^{T=0}, A_{0}^{T=0}=A^{T=0}$ are the solutions to the gap equation at zero temperature.

Given that we have already solved the systems at zero temperature, as detailed in the last chapter, it may seem that the computation would be straight forward. This is unfortunately not the case. Recall that the expression of $\Pi_{1}$ alone is divergent, while the finite, renormalized $\Pi_{1}^{R}$ depends on the cancellation of two divergences of the two full fermion propagators. However, in the process of obtaining the solutions through iteration, the intermediate trial solutions fail to honor this quality. At the present time, we can only proceed by imposing further simplification, for example: setting the electric screening mass to its perturbative expression. Even under this simplification, the gap equations still admit feedback between photonic and fermionic sectors. To be concrete, recall the renormalized electric polarization scalar $\Pi_{1}$ is given by:

$$
\begin{aligned}
\Pi_{1}{ }^{R}\left[B, A, A_{0}\right]\left(\omega_{n}, \vec{k}\right)= & \Pi_{1}\left[B, A, A_{0}\right]\left(\omega_{n}, \vec{k}\right)-\Pi_{1}^{T=0}\left[B^{T=0}, A^{T=0}, A_{0}^{T=0}=A^{T=0}\right](0,0) \\
= & {\left[\Pi_{1}\left[B, A, A_{0}\right]\left(\omega_{n}, \vec{k}\right)-\Pi_{1}\left[B, A, A_{0}\right](0,0)\right]+} \\
& {\left[\Pi_{1}\left[B, A, A_{0}\right](0,0)-\Pi_{1}^{T=0}\left[B^{T=0}, A^{T=0}, A_{0}^{T=0}=A^{T=0}\right](0,0)\right] } \\
= & {\left[\Pi_{1}\left[B, A, A_{0}\right]\left(\omega_{n}, \vec{k}\right)-\Pi_{1}\left[B, A, A_{0}\right](0,0)\right]+m_{\mathrm{el}}^{R} . }
\end{aligned}
$$

It is clear that even if one replaces $m_{\mathrm{el}}^{R} \longrightarrow m_{\mathrm{el}}^{P}$, the system of the integral equations is still strongly coupled. 


\subsection{NUMERICS AND RESULTS}

The massless, perturbative limit of the electric screening mass at finite temperature is given in section 5.3.1. The correspond sum at finite density and temperature can be analogously computed, the result reads:

$$
\Pi_{1 \mathrm{mat}}^{P}[0,0]=m_{\mathrm{el}}^{P}=2 \alpha T\left\{\left[2 \ln \left[2 \cosh \frac{x_{0}-x_{\mu}}{2}\right]-x_{0} \tanh \left[\frac{x_{0}-x_{\mu}}{2}\right]\right]+x_{\mu} \rightarrow-x_{\mu}\right\},
$$

with

$$
\begin{aligned}
& x_{0}=\beta M \\
& x_{\mu}=\beta \mu .
\end{aligned}
$$

For our present study, we will pick the massless limit:

$$
m_{\mathrm{el}}^{P}[T, \mu]=8 \alpha T\left\{\ln \left[e^{\frac{x_{\mu}}{2}}+e^{-\frac{x_{\mu}}{2}}\right]\right\}
$$

Employing this expression in place of the full electric mass, we set out to compute the phase map for the fully quenched system of QED3 ${ }^{2}$. The finite temperature expression for the condensate reads:

$$
\langle\bar{\psi} \psi\rangle=\frac{1}{\beta} \sum_{\omega_{n}^{\prime}} \int \frac{d^{2} k^{\prime}}{2 \pi)^{2}}(\operatorname{Tr}[I]) \frac{-B}{A^{0}\left(i \omega_{n}^{\prime}+\mu\right)^{2}-A^{2}{k^{\prime}}^{2}-B^{2}} .
$$

where $\operatorname{Tr}[I]=4$ for 4 -spinor representation.

The major numerical techniques employed are similar to the respective zero temperature study, only that the Matsubara frequencies and the spatial momentums have to be treated as independent degrees of freedom, which taxes heavily on the computer resources required for a self-consistent calculation. The results below represent months of numerical efforts.

\footnotetext{
${ }^{2}$ We shall again work in Landau gauge, the infrared divergence from the magnetic polarization scalar is regulated by an infrared cutoff $\epsilon_{I R}$. The energy scale is measured in unit of $\alpha$.
} 


\subsubsection{QUENCHED CASE}

For the quenched calculation, we neglect the contribution from the photon polarization. The fermion self-energy equation should be regulated by an infrared cutoff, which we choose to be $\epsilon_{I R}=0.1^{3}$. Shown below is the phase map for the quenched system of QED3 at finite temperature and density:

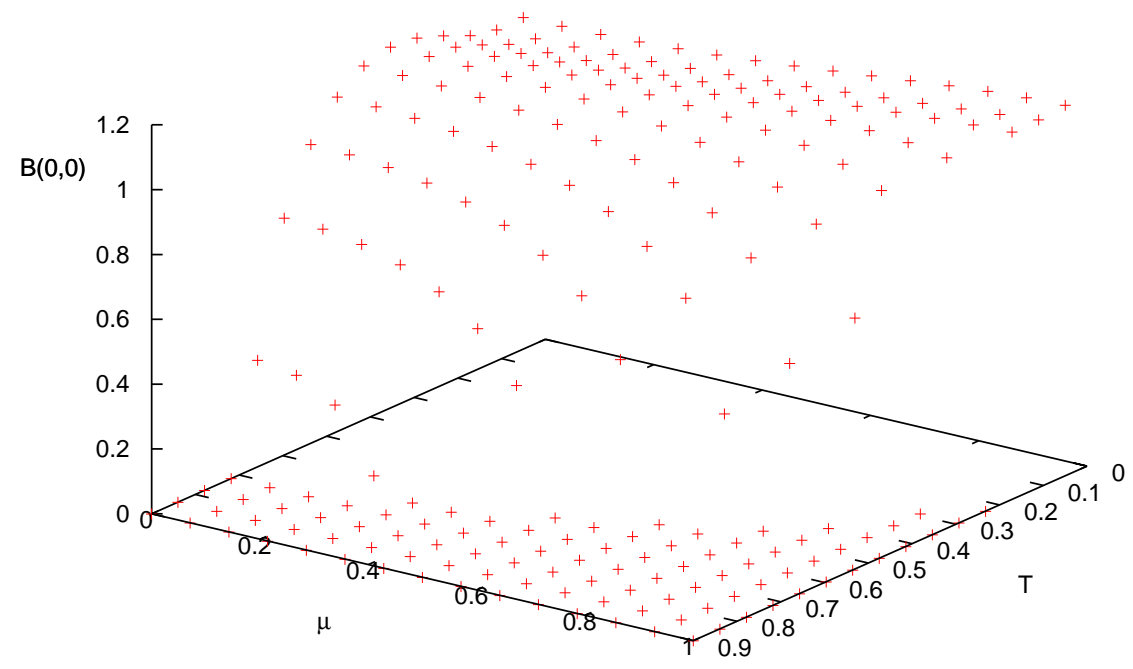

Figure 26: Phase diagram for quenched QED3 in RL approximation. The phase transitions are 2nd order in nature. $\epsilon_{I R}=0.1$

\footnotetext{
${ }^{3}$ The infrared regulator enters the expression of propagator for photon as $\frac{1}{k^{2}+\epsilon_{I R}}$. It is measured in units of $\alpha^{2}$
} 


\subsubsection{UNQUENCHED CASE}

Our first step of unquenching the gap equations involve the use of static, massless and perturbative expression for the ring, namely:

$$
\Pi_{1}^{R}\left[\omega_{n}, k\right]=m_{\mathrm{el}}^{P}=2 \alpha T\left\{\left[2 \ln \left[2 \cosh \frac{x_{0}-x_{\mu}}{2}\right]-x_{0} \tanh \left[\frac{x_{0}-x_{\mu}}{2}\right]\right]+x_{\mu} \rightarrow-x_{\mu}\right\} .
$$

The resulting phase map is shown below:

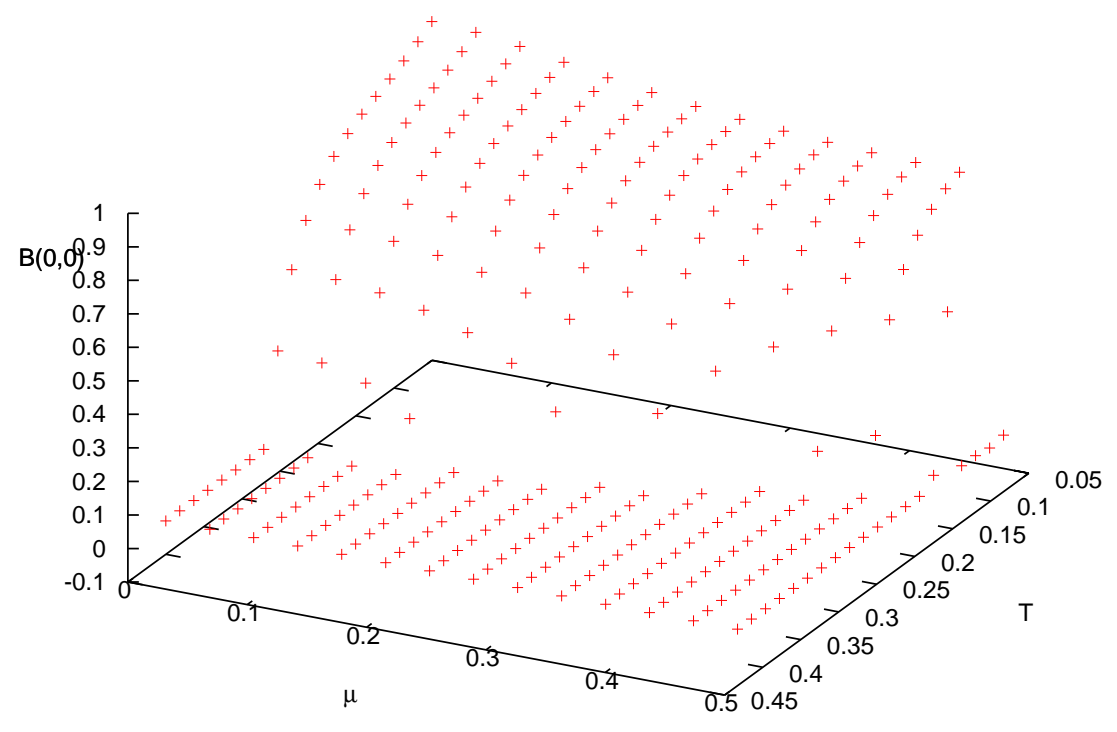

Figure 27: Phase diagram for QED3 with static perturbative ring. The phase transitions are 2 nd order in nature. $\epsilon_{I R}=0.1$ 
We can improve the situation by including the momentum dependent non-perturbative contributions, renormalized in the simplified limit motivated in the previous section:

$$
\begin{aligned}
\Pi_{1}{ }^{R}\left[B, A, A_{0}\right]\left(\omega_{n}, \vec{k}\right)= & \Pi_{1}\left[B, A, A_{0}\right]\left(\omega_{n}, \vec{k}\right)-\Pi_{1}^{T=0}\left[B^{T=0}, A^{T=0}, A_{0}^{T=0}=A^{T=0}\right](0,0) \\
= & {\left[\Pi_{1}\left[B, A, A_{0}\right]\left(\omega_{n}, \vec{k}\right)-\Pi_{1}\left[B, A, A_{0}\right](0,0)\right]+} \\
& {\left[\Pi_{1}\left[B, A, A_{0}\right](0,0)-\Pi_{1}^{T=0}\left[B^{T=0}, A^{T=0}, A_{0}^{T=0}=A^{T=0}\right](0,0)\right] } \\
= & {\left[\Pi_{1}\left[B, A, A_{0}\right]\left(\omega_{n}, \vec{k}\right)-\Pi_{1}\left[B, A, A_{0}\right](0,0)\right]+m_{\mathrm{el}}^{R} }
\end{aligned}
$$

with the simplification

$$
m_{\mathrm{el}}^{R} \approx m_{\mathrm{el}}^{P}
$$

This represents our most sophisticated truncation for the system, showing below is the phase map for such system:

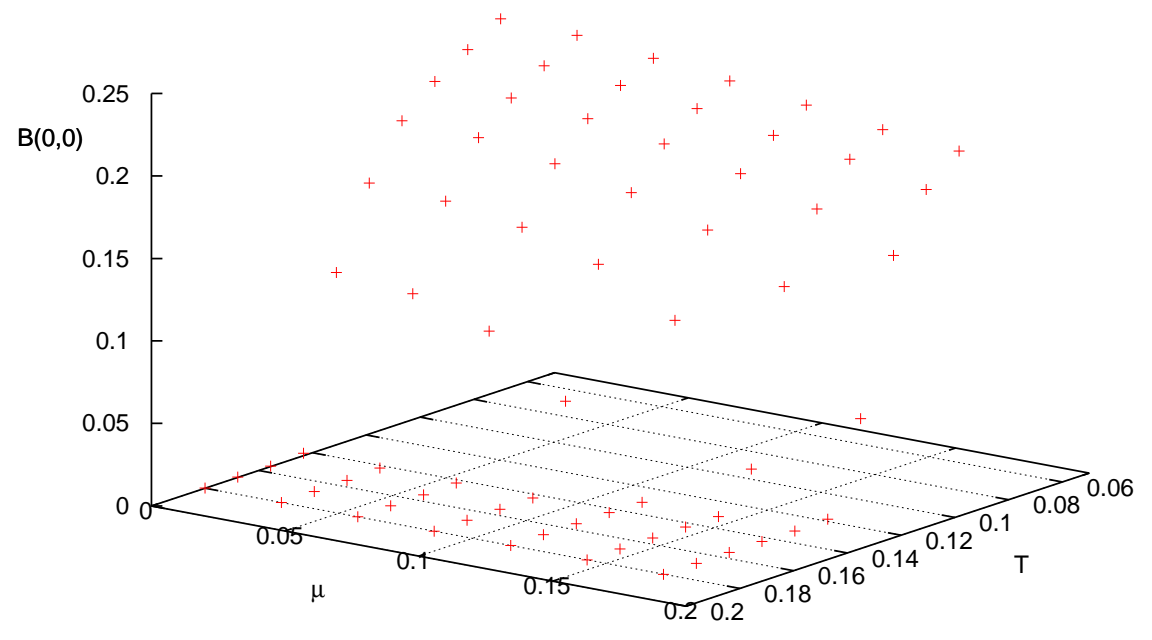

Figure 28: Phase diagram for QED3 in RL approximation. The phase transitions are 2nd order in nature. $\epsilon_{I R}=0.1$ 
We observe a second order phase transition for the range of $\mu$ we studied.

It is worth remembering that the expected gauge invariance can only be imperfectly realized in the above cases. The reasons are as follow: firstly, the rainbow ladder truncation employed here does not respect the Ward identity: the transversality of the polarization tensor is at stake because of the non-ideal truncation. Also, the simplification used in the non-perturbative renormalization is expected to worsen the situation. Last, with any truncation, even the gauge invariant one, the solution obtained would differ from the true solution of the system. One possible diagnostic is the Coleman-Hill theorem, generalized naturally to finite temperature. Progress along this line of thought is underway.

\subsection{CONCLUSIONS}

We have extended the study of dynamical chiral symmetry breaking in QED3 to finite temperature and density.

The theory contains an endemic infrared divergence in the fermion self energy, which is absent in the zero temperature version. The origin of this infrared divergence is closely related to the absence of magnetic mass, true to all orders. However, with the observation that the divergence takes the same form as the pure gauge term, we argue that physical quantities should be safe from this infrared divergence.

The method of non-perturbative renormalization for the electric screening mass is proposed. Unfortunately, implementation of the full expression to the system of coupled integral equations presents serious numerical challenges. We discuss one further simplification as the temporary resort, namely, the use of the perturbative expression for the screening mass, while the momentum dependent part remains fully non-perturbative. An unquenched calculation, with Matsubara frequency and momentum dependent photonic and fermionic propagators, is then attempted.

The phase map for the chiral symmetry breaking in the simplified case is presented. It is found that the phase transitions are second order. The study demonstrates the possibility of solving a full Schwinger Dyson systems at finite temperature and density. However, 
gauge invariance can only be imperfectly realized due to the use of the rainbow ladder truncation and the simplification made in the non-perturbative renormalization. Moreover, from experience, the phase structure can change drastically if one considers the full nonperturbative electric mass, such a study is currently underway. 


\subsection{CONCLUSIONS}

In this thesis we have studied various confinement models at finite temperature and density, focusing on the aspects of dynamical chiral and parity symmetry breaking. Schwinger Dyson equations, under various degrees of truncations, are employed as the tool to extract nonperturbative information.

We started by investigating two types of static confinement models motivated by Coulomb gauge QCD: contact and linear potentials.

For the contact potential, we present the phase map for both the bare and ring versions. The bare contact model exhibits many interesting features such as the existence of tricritical point and the approximate linear scaling of $T_{c}, \mu_{c}$ with the coupling. The numerical values of the constituent mass, condensate, critical temperature and chemical potential can be brought into rough agreement with the physical expectations in QCD by an appropriate choice of parameters. However, the incorporation of polarization effects drastically affects the phase structure. The most prominent effects are the substantial drop in the critical temperature (ruining phenomenology) and the dominance of first order phase transition.

For the linear potential, both the AAL prescription for the bare potential and the ring approximation yield a chiral phase transition, contrary to the expectation of Davis and Matheson. Especially, given the fact that large $N_{c}$ limit tends to suppress quark loop effects, one can argue the bare linear potential studied here is an implementation of the large $N_{c}$ scenario. Our results then support the idea of quarkyonic matter argued by Pisarski and

McLerran [37]: the existence of confining but chirally symmetric phase. The summation of ring diagrams is motivated as the resolution of the infrared divergence problem in the linear potential. The numerical values for the constituent mass, condensate, critical temperature and density can attain reasonable QCD limit if the string tension $b$ is increased to $1.8 \mathrm{GeV}$. 
Unfortunately this is at odds with the well-established quark model phenomenology and lattice data, which requires $b=0.2 \mathrm{GeV}$. Therefore, it appears that naive static potential model fail to describe the thermal properties of QCD.

We then turned our attention to the study of QED3.

For the case of zero temperature QED3, we studied dynamical parity and chiral symmetry breaking within 2-spinor formalism. Schwinger-Dyson equations with various truncations, including the Ward identity preserving BC and CP vertex. The Coleman-Hill theorem is employed to check the robustness of the truncations.

Parity violating solutions are found for $\eta \lesssim 0.4$, while no solution can be found for the case of maximal violation $(\eta=1)$. It is interesting to note that the possibility of any parityviolating solution is at odds with the traditional view [52], this point certainly deserves further investigation.

Large ratios of mass scales are dynamically generated as one varies $N_{f}$. Critical $N_{f}$ for chiral restoration are reasonably close to that obtained by Appelquist et al., which is given by $N_{\star}^{A}=\frac{64}{\pi^{2}}$.

The Coleman-Hill theorem is used as a diagnostic for truncation accuracy. It verifies the intuition that truncations which obey the Ward identity should perform better than those that are not.

The study is extended to finite temperature and density. The theory contains an endemic infrared divergence in the fermion self energy, which is absent in the zero temperature version. The origin of this infrared divergence is closely related to the absence of magnetic mass, true to all orders. However, with the observation that the divergence takes the same form as the pure gauge term, we argue that physical quantities should be safe from this infrared divergence.

The method of non-perturbative renormalization for the electric screening mass is proposed. However, implementation of the full expression to the system of coupled integral equations presents serious numerical challenges. We discuss one further simplification as the temporary resort, namely, the use of the perturbative expression for the screening mass, while the momentum dependent part remains fully non-perturbative. An unquenched calculation, with Matsubara frequency and momentum dependent photonic and fermionic propagators, 
is then attempted.

The phase map for the chiral symmetry breaking in the simplified case is presented. It is found that the phase transitions are second order. The study demonstrates the possibility of solving a full Schwinger Dyson systems at finite temperature and density. However, gauge invariance can only be imperfectly realized due to the use of the rainbow ladder truncation and the simplification made in the non-perturbative renormalization. Moreover, from experience, the phase structure can change drastically if one considers the full nonperturbative electric mass, such a study is currently underway.

In studying the various models, we have employed a diversity of truncation schemes for the Schwinger Dyson equations. One may reasonably question whether any truncation made can be justified. After all, in a non-perturbative formulation, one does not have a controlled expansion for physical quantity in series of a small parameter. Here we try to address this issue in several levels.

Within the framework of Schwinger Dyson equations, one can study a specific problem using different truncations and check for internal consistencies. One can also design some benchmark tests (like the Coleman-Hill theorem) to gauge the efficacy of the method.

Another important justification is by cross-checking with alternative non-perturbative methods. By studying a given model (like QED3) in parallel with other approaches (like $1 / N$ expansion, lattice, etc), one can gain confidence in the method when the results obtained are consistent with one another.

Finally, we stress that the ultimate judge on the merit of a calculation scheme should be based on the comparison of its predictions with physical experiments. This reflects our pragmatic stance on the issue, and in fact, on doing theoretical physics in general. 
APPENDIX A

\section{CONVENTIONS}

\section{A.1 METRICS AND GAMMA MATRICES}

Our convention for the signature of the metric is $(1,-\vec{I})$

$$
g^{\mu \nu}=\left(\begin{array}{cccc}
1 & 0 & 0 & 0 \\
0 & -1 & 0 & 0 \\
0 & 0 & -1 & 0 \\
0 & 0 & 0 & -1
\end{array}\right)
$$

The Pauli matrices are defined by

$$
\begin{gathered}
\sigma_{x}=\left(\begin{array}{ll}
0 & 1 \\
1 & 0
\end{array}\right) \\
\sigma_{y}=\left(\begin{array}{cc}
0 & -i \\
i & 0
\end{array}\right) \\
\sigma_{z}=\left(\begin{array}{cc}
1 & 0 \\
0 & -1
\end{array}\right) .
\end{gathered}
$$


The gamma matrices are defined as

$$
\begin{gathered}
\gamma^{0}=\left(\begin{array}{cc}
0 & \vec{I} \\
\vec{I} & 0
\end{array}\right) \\
\vec{\gamma}=\left(\begin{array}{cc}
0 & \vec{\sigma} \\
-\vec{\sigma} & 0
\end{array}\right)
\end{gathered}
$$

They satisfy the Dirac algebra

$$
\left\{\gamma^{\mu}, \gamma^{\nu}\right\}=2 g^{\mu \nu}
$$

Note that $\gamma_{5}$ can be defined for 4 -spinor space as

$$
\gamma_{5}=\left(\begin{array}{cc}
\vec{I} & 0 \\
0 & -\vec{I}
\end{array}\right)
$$

satisfying

$$
\left\{\gamma^{\mu}, \gamma_{5}\right\}=0
$$




\section{A.2 EUCLIDEAN SPACE CONVENTIONS}

To convert from Minkowski space to Euclidean space, we write

$$
\begin{aligned}
& x^{0} \longrightarrow-i x_{4} \\
& k^{0} \longrightarrow i k_{4},
\end{aligned}
$$

such that

$$
\begin{aligned}
\int_{\text {Minkowski }} d^{4} x f\left[x^{0}, \vec{x}\right] & \longrightarrow-i \int_{\text {Euclidean }} d^{4} x_{E} f\left[-i x_{4}, \vec{x}\right] \\
\int_{\text {Minkowski }} d^{4} k f\left[k^{0}, \vec{k}\right] & \longrightarrow i \int_{\text {Euclidean }} d^{4} k_{E} f\left[i k_{4}, \vec{k}\right] \\
a \cdot b & \longrightarrow-a_{E} \cdot b_{E}
\end{aligned}
$$




\section{APPENDIX B}

\section{INTRODUCTION TO FUNCTIONAL METHODS IN QFT}

Functional methods prove to be essential in the study of QFT [1, 3, 61]. Not only does it provides an economical formalism for deriving familiar results, such as the Schwinger-Dyson equations, it also yield valuable insight into the study of subtle properties like collective phenomena and symmetry in QFT.

A functional maps a function in function space (the infinite dimensional space with each "point" being a function on space time) into a c-number. As we move from function to function, we get a different c-number through the functional, this motivates the definition of functional differentiation

$$
\frac{\delta F[f[x]]}{\delta f(x)}=\lim _{\epsilon \rightarrow 0} \frac{F[f[x]+\epsilon]-F[x]}{\epsilon}
$$

In particular, note that

$$
\frac{\delta \phi_{x}}{\delta \phi_{y}}=\delta[x-y]
$$




\section{B.1 CENTRAL IDENTITIES OF QFT}

We define delta-functional $\delta\left[\phi-\phi_{0}\right]$ to be $\prod_{x} \delta\left[\phi_{x}-\phi_{0 x}\right]$ so that

$$
\int D \phi F[\phi] \delta\left[\phi-\phi_{0}\right]=F\left[\phi_{0}\right]
$$

for any functional $F[\phi]$.

We also note the relation

$$
\delta\left[\phi_{0}\right]=\int D A e^{i \int A \phi_{0}}
$$

Next, we turn to functional integral involving quadratics. The results of Gaussian integral in calculus have their counterparts in the Gaussian functional integral:

$$
\begin{aligned}
\int d x e^{-a x^{2} \pm b x} & =\sqrt{\frac{\pi}{a}} e^{\frac{b^{2}}{4 a}} \\
\int d z d z^{\star} e^{-a|z|^{2}} & =\frac{\pi}{a} \\
\int D \phi e^{i\left[\int \phi D \phi+j \phi\right]} & =\sqrt{\frac{\pi^{\infty}}{(-i)^{\infty} \operatorname{Det}[D]}} e^{-i \frac{1}{4} \int j D^{-1} j} \\
\int D \phi^{\star} D \phi e^{i\left[\int \phi^{\star} D \phi+j^{\star} \phi+j \phi^{\star}\right]} & =\frac{\pi^{\infty}}{(-i)^{\infty} \operatorname{Det}[D]} e^{-i \int j^{\star} D^{-1} j}
\end{aligned}
$$

While for fermionic functional integral, one gets

$$
\int D \bar{\psi} D \psi e^{i \int \bar{\psi} M \psi+\bar{\eta} \psi+\bar{\psi} \eta}=i^{N(N+2)} \operatorname{Det}[M] e^{-i \int \bar{\eta} M^{-1} \eta}
$$

where $N$ is the dimension of the functional space, usually infinite

For all practical calculations, we will drop all uninteresting constant factors and use the following: 


$$
\begin{aligned}
\int D \phi e^{i\left[\int \phi D \phi+j \phi\right]} & =\sqrt{\frac{1}{\operatorname{Det}[D]}} e^{-i \frac{1}{4} \int j D^{-1} j} \\
\int D \phi^{\star} D \phi e^{i\left[\int \phi^{\star} D \phi+j^{\star} \phi+j \phi^{\star}\right]} & =\frac{1}{\operatorname{Det}[D]} e^{-i \int j^{\star} D^{-1} j} \\
\int D \bar{\psi} D \psi e^{i \int \bar{\psi} M \psi+\bar{\eta} \psi+\bar{\psi} \eta} & =\operatorname{Det}[M] e^{-i \int \bar{\eta} M^{-1} \eta}
\end{aligned}
$$

A useful identity to handle determinant is

$$
\operatorname{Det}[M]=e^{T r \ln M}
$$

\section{B.2 FUNCTIONAL APPROACH TO FREE FIELD THEORY}

We shall illustrate the essence of functional methods with free fields calculations. Of course the results should be familiar, nevertheless we take this chance to clarify our notation of the various N-point functions.

\section{B.2.1 SCALARS}

The Lagrangian density is given by

$$
\mathcal{L}=\frac{1}{2}(\partial \phi)^{2}-\frac{1}{2} m^{2} \phi^{2} .
$$

From this, one can construct the generating functional

$$
Z[j]=\int D \phi e^{i \int-\frac{1}{2} \phi\left(\partial^{2}+m^{2}\right) \phi+j \phi}
$$

Realizing $D=-\frac{1}{2}\left(\partial^{2}+m^{2}\right)$ and applying B.8: 


$$
Z[j]=\sqrt{\frac{1}{\operatorname{Det}[D]}} e^{i \frac{1}{2} \int j \frac{1}{\partial^{2}+m^{2}} j}
$$

The generating functional for connected Green's function is defined as $W=-i \ln Z$. One obtains

$$
W[j]=\frac{1}{2} \int j \frac{1}{\partial^{2}+m^{2}} j+\text { Constant }
$$

Finally the generating functional for 1PI Green's function is defined as $\Gamma=W-\int j[\phi] \phi$, where $j[\phi]=\frac{\delta W}{\delta j}$. In this case, we can invert $\phi$ from $j[\phi]$ easily

$$
\phi=\frac{1}{\partial^{2}+m^{2}} j .
$$

Therefore, one reaches an exact expression for $\Gamma[\phi]$ :

$$
\Gamma[\phi]=-\frac{1}{2} \int \phi\left(\partial^{2}+m^{2}\right) \phi=\int \mathcal{L} .
$$

Performing the functional differentiation in this case is trivial:

$$
\begin{aligned}
(-i)^{2} \frac{\delta^{2}}{\delta j \delta j} \frac{Z[j]}{\left.Z[0]\right|_{j \rightarrow 0}} & =\frac{-i}{\partial^{2}+m^{2}} \delta \leftrightarrow \frac{i}{p^{2}-m^{2}} \\
\frac{\delta^{2} W}{\delta j \delta j} & =\frac{1}{\partial^{2}+m^{2}} \delta \leftrightarrow \frac{-1}{p^{2}-m^{2}} \\
\frac{\delta^{2} \Gamma}{\delta \phi \delta \phi} & =-\left(\partial^{2}+m^{2}\right) \delta \leftrightarrow p^{2}-m^{2}
\end{aligned}
$$

In the standard notation [5], one associates

$$
\langle 0|T\{\phi, \phi\}| 0\rangle=D_{F}=\int \frac{d^{4} p}{(2 \pi)^{4}} \frac{i}{p^{2}-m^{2}+i \epsilon} e^{-i p \cdot x} .
$$

Defining $\langle 0|T\{\phi, \phi\}| 0\rangle=i \Delta_{F}$, we identify 


$$
\begin{aligned}
\left.(-i)^{2} \frac{\delta^{2}}{\delta j \delta j} \frac{Z[j]}{Z[0]}\right|_{j \rightarrow 0} & =i \Delta_{F} \\
\frac{\delta^{2} W}{\delta j \delta j} & =-\Delta_{F} \\
\frac{\delta^{2} \Gamma}{\delta \phi \delta \phi} & =\Delta_{F}^{-1}
\end{aligned}
$$

\section{B.2.2 FERMIONS}

Now we turn to the illustration with free fermions. To account for the anti-commuting nature of the fermionic fields, one introduces the Grassmann numbers satisfying

$$
\left\{\eta, \eta^{\prime}\right\}=0
$$

while they are commuting with normal c-number.

In particular, we observe that $\eta^{2}=0$, hence, a Taylor expansion of a Grassmannian function $F[\eta]$ can only contain two terms

$$
F[\eta]=a+b \eta
$$

where $a, b$ are c-numbers.

The table for the Grassmannian integration is short, in fact, it has only two entries

$$
\begin{aligned}
& \int d \eta 1=0 \\
& \int d \eta \eta=1
\end{aligned}
$$

consistent with the condition

$$
\int d \eta \frac{d}{d \eta} F[\eta]=0
$$

With these properties, one can derive the result 


$$
\int D \bar{\psi} D \psi e^{i \int \bar{\psi} M \psi+\bar{\eta} \psi+\bar{\psi} \eta}=\operatorname{Det}[M] e^{-i \int \bar{\eta} M^{-1} \eta}
$$

Consider the free fermion Lagrangian

$$
\mathcal{L}=\bar{\psi}(i \not \partial-m) \psi
$$

With B.8, one immediately obtains

$$
Z=\int D \bar{\psi} D \psi e^{i \int \mathcal{L}+\bar{\eta} \psi+\bar{\psi} \eta}=\operatorname{Det}[i \not \partial-m] e^{-i \int \bar{\eta} \frac{1}{(i \bar{\phi}-m)} \eta}
$$

A straight forward calculation gives

$$
\begin{gathered}
W=-i \ln Z=-\int \bar{\eta} \frac{1}{(i \not \partial-m)} \eta+C \\
\Gamma=S[\psi, \bar{\psi}]=\int \bar{\psi}(i \not \partial-m) \psi .
\end{gathered}
$$

Again, the usual convention for the propagator reads

$$
\langle 0|T\{\psi, \bar{\psi}\}| 0\rangle=D_{F}=\int \frac{d^{4} p}{(2 \pi)^{4}} \frac{i}{\not p-m+i \epsilon} e^{-i p \cdot x}=i \Delta_{F} .
$$

We identify

$$
\begin{aligned}
\left.(-i)^{2} \frac{\delta^{2}}{\delta j \delta j} \frac{Z[j]}{Z[0]}\right|_{j \rightarrow 0} & =i \Delta_{F} \\
\frac{\delta^{2} W}{\delta \bar{\eta} \delta \eta} & =\Delta_{F} \\
\frac{\delta^{2} \Gamma}{\delta \bar{\psi} \delta \psi} & =-\Delta_{F}^{-1} .
\end{aligned}
$$




\section{B.2.3 GAUGE FIELDS}

The abelian Yang-Mills Lagrangian density reads

$$
\mathcal{L}_{Y M}=-\frac{1}{4} F^{2}=\frac{1}{2} A_{\mu}\left(\partial^{2} g^{\mu \nu}-\partial^{\mu} \partial^{\nu}\right) A^{\nu} .
$$

Writing $\hat{O}=\frac{1}{2}\left(\partial^{2} g^{\mu \nu}-\partial^{\mu} \partial^{\nu}\right)$, one may attempt to proceed as usual to define

$$
Z[j]=\int D A e^{i \int \frac{1}{2} A_{\mu}\left(\partial^{2} g^{\mu \nu}-\partial^{\mu} \partial^{\nu}\right) A^{\nu}+j \cdot A}=\sqrt{\frac{1}{\operatorname{Det}[\hat{O}]}} e^{-i \frac{1}{2} \int j \frac{1}{\partial^{2} g^{\mu \nu}-\partial^{\mu} \partial^{\nu}} j} .
$$

However, due to the existence of zero eigenvalues for $\hat{O}$, the inverse is ill-defined. In fact, the inverse formally diverges. The divergence has its origin in gauge symmetry and we will expand on the topic of gauge fixing in section B.3. For our present purpose, we just mention the procedure of covariant gauge fixing involve the addition of the term $-\frac{1}{2 \xi}\left(\partial_{\mu} A^{\mu}\right)^{2}$ to $\mathcal{L}_{Y M}$ and hence the generating functional becomes

$$
Z[j]=\int D A e^{i \int \frac{1}{2} A_{\mu}\left(\partial^{2} g^{\mu \nu}-\left(1-\xi^{-1}\right) \partial^{\mu} \partial^{\nu}\right) A^{\nu}+j \cdot A}=Z[0] e^{-i \frac{1}{2} \int j \frac{1}{\partial^{2} g^{\mu \nu}-\left(1-\xi^{-1}\right) \partial^{\mu} \partial^{\nu}}} .
$$

Now the inverse is well defined and one can work out the solution

$$
\left[\partial^{2} g^{\mu \rho}-\partial^{\mu} \partial^{\rho}\left(1-\xi^{-1}\right)\right] \int \frac{d^{4} k}{(2 \pi)^{4}} e^{-i k \cdot x} \frac{-\left[g_{\rho \nu}-(1-\xi) \frac{k_{\rho} k_{\nu}}{k^{2}}\right]}{k^{2}}=g_{\nu}^{\mu} \delta
$$

Denote

$$
D_{\mu \nu}^{A}=\int \frac{d^{4} k}{(2 \pi)^{4}} e^{-i k \cdot x} \frac{-\left[g_{\mu \nu}-(1-\xi) \frac{k_{\mu} k_{\nu}}{k^{2}}\right]}{k^{2}},
$$

and again $\langle 0|T\{A, A\}| 0\rangle=i D_{A}$, we make the connections

$$
\begin{aligned}
\left.(-i)^{2} \frac{\delta^{2}}{\delta j \delta j} \frac{Z[j]}{Z[0]}\right|_{j \rightarrow 0} & =i D_{A} \\
\frac{\delta^{2} W}{\delta j \delta j} & =-D_{A} \\
\frac{\delta^{2} \Gamma}{\delta A \delta A} & =D_{A}{ }^{-1} .
\end{aligned}
$$




\section{B.3 GAUGE FIXING FOR YANG-MILLS THEORY}

The need for gauge fixing for Yang-Mills theory stems from the gauge symmetry. To see this, consider the generating functional for the abelian case

$$
Z=\int D A e^{i \int \mathcal{L}[\mathcal{A}]}
$$

where $\mathcal{L}$ is invariant under the gauge transformation $A \rightarrow A+\partial \alpha$. Then we can split the functional integral into

$$
Z=\int D A^{\prime} D \alpha\left[A^{\prime}\right] e^{i \int \mathcal{L}\left[\mathcal{A}^{\prime}\right]},
$$

with various $A^{\prime}$ not related by the gauge transformation, corresponding to all physically distinct configurations, while $\alpha$ being all possible gauge transformation. The integrand is $\alpha$-independent as dictated by gauge symmetry, it is now clear that

$$
Z=\int D A^{\prime} e^{i \int \mathcal{L}\left[\mathcal{A}^{\prime}\right]} \int D \alpha\left[A^{\prime}\right]
$$

is divergent due to the unphysical integral of gauge volume $\int D \alpha$.

Gauge fixing is the selection of a representative element $A^{\prime}$ from the orbit of all configurations related by the gauge transformation (the gauge orbit), it is performed by the gauge condition

$$
G\left[A^{\prime}\right]=0 .
$$




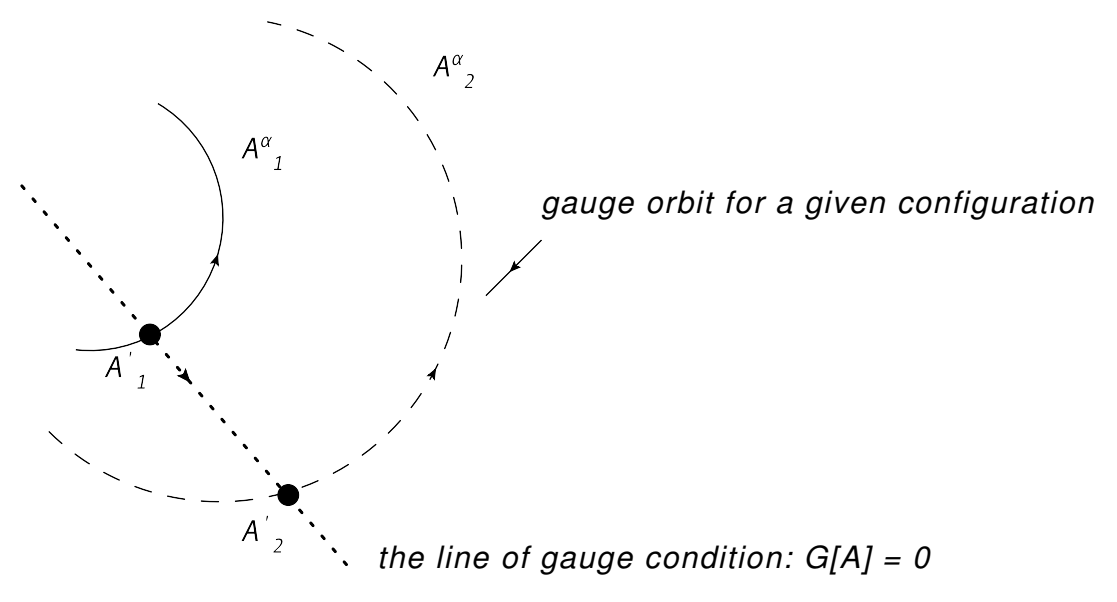

Figure 29: Illustration of gauge orbits and gauge condition: The gauge condition selects one representative element from each gauge orbit.

Making use of the delta functional and the relation

$$
\delta[\alpha-\alpha[G]]=\delta[G]\left|\operatorname{Det}\left[\frac{\delta G[\alpha]}{\delta \alpha}\right]\right|,
$$

one obtains the refined version of the generating functional

$$
\begin{aligned}
Z_{G F} & =\int D A^{\prime} D \alpha \delta[\alpha-\alpha[G]] e^{i \int \mathcal{L}\left[\mathcal{A}^{\prime}\right]} \\
& =\int D A \delta[G]\left|\operatorname{Det}\left[\frac{\delta G}{\delta \alpha}\right]\right| e^{i \int \mathcal{L}[\mathcal{A}]} .
\end{aligned}
$$

The above implementation of gauge fixing is just a restatement to the Faddeev-Popov procedure.

Some famous gauge fixing conditions are:

- Coulomb gauge: $G[A]=\vec{\nabla} \cdot \vec{A}$

- Lorentz gauge: $G[A]=\partial_{\mu} A^{\mu}$

- General $\xi$-gauge: $G[A]=\partial_{\mu} A^{\mu}-c_{\xi}$ 
In employing the $\xi$-gauge, it is helpful to consider a slightly modified version of the generating functional

$$
\begin{aligned}
Z_{G F} & =\int D A D c[x] \delta\left[\partial_{\mu} A^{\mu}-c[x]\right]\left|\operatorname{Det}\left[\frac{\delta G}{\delta \alpha}\right]\right| e^{-i \frac{1}{2 \xi} \int c^{2}} e^{i \int \mathcal{L}[\mathcal{A}]} \\
& =\int D A \operatorname{Det}\left[\partial^{2}\right] e^{i \frac{1}{2} \int A\left[\partial^{2} g^{\mu \nu}-\partial^{\mu} \partial^{\nu}\left(1-\xi^{-1}\right)\right] A}
\end{aligned}
$$

where on the second line, we have explicitly used the Lagrangian of the abelian Yang-Mills theory. Also, $\operatorname{Det}\left[\partial^{2}\right]$ in this case is independent of the field $A$ and hence may be dropped for practical calculations.

\section{B.3.1 COULOMB GAUGE IN QED}

We now review the process of gauge fixing in Coulomb gauge QED. The absence of the term $\partial_{t} A^{0}$ in the Lagrangian means that $A^{0}$ is non-dynamical, and can be functionally integrated away, as we shall see, this leads to the explicit appearance of the Coulomb potential in the Lagrangian.

$$
\mathcal{L}_{Q E D}=\bar{\psi}(i \not \supset-m-g \not) \psi-\frac{1}{4} F^{2}
$$

where $F_{\mu \nu}=\partial_{\mu} A_{\nu}-\partial_{\nu} A_{\mu}$.

The proper generating functional is given by

$$
Z=\int D \bar{\psi} D \psi D A \delta[\vec{\nabla} \cdot A] \operatorname{Det}\left[-\vec{\nabla}^{2}\right] e^{i \int \bar{\psi}(\not \not \supset-m-g \AA) \psi-\frac{1}{4} F^{2}} .
$$

Noting the definition of electric field and magnetic field

$$
\begin{aligned}
E^{i} & =F^{i 0}=\partial^{i} A^{0}-\partial_{t} A^{i} \\
B^{i} & =-\frac{1}{2} \epsilon^{i j k} F_{j k},
\end{aligned}
$$

one can work out 


$$
-\frac{1}{4} F^{2}=\frac{1}{2}\left(\vec{E}^{2}-\vec{B}^{2}\right)
$$

Focus on the term

$$
e^{i \int \frac{1}{2} F^{i 0} F^{i 0}}=\int D \vec{\Pi} e^{i \int-\frac{1}{2} \Pi^{i} \Pi^{i}+\Pi^{i} F^{i 0}}
$$

the generating functional is rewritten as

$$
\begin{aligned}
& Z=\int D \bar{\psi} D \psi D \vec{A} D A^{0} D \vec{\Pi} \delta[\vec{\nabla} \cdot A] \operatorname{Det}\left[-\vec{\nabla}^{2}\right] \\
& e^{i \int \bar{\psi}(i \not \partial-m) \psi+g \bar{\psi} \vec{\gamma} \psi \cdot \vec{A}+\vec{\Pi} \cdot\left[\partial_{t} \vec{A}\right]-\frac{1}{2} \vec{B}^{2}-\frac{1}{2} \vec{\Pi}^{2}+\left[-\vec{\Pi} \cdot \vec{\nabla}-g \bar{\psi} \gamma^{0} \psi\right] A^{0} .}
\end{aligned}
$$

One can remove the explicit $A^{0}$-degree of freedom by the functional integration:

$$
\int D A^{0} e^{i \int\left[-\vec{\Pi} \cdot \vec{\nabla}-g \bar{\psi} \gamma^{0} \psi\right] A^{0}}=\delta\left[\vec{\nabla} \cdot \vec{\Pi}-g \bar{\psi} \gamma^{0} \psi\right]
$$

Rewriting $\vec{\Pi}$ as

$$
\vec{\Pi}=-\vec{\nabla} \phi+\overrightarrow{\Pi^{\perp}}
$$

where $\vec{\nabla} \cdot \overrightarrow{\Pi^{\perp}}=0$. Note that $\frac{1}{2} \vec{\Pi}^{2}=\frac{1}{2} \vec{\Pi}^{2}+\frac{1}{2} \phi\left(-\vec{\nabla}^{2}\right) \phi$.

The functional integral of $\vec{\Pi}$ can be decomposed into

$$
D \vec{\Pi} \longrightarrow D \vec{\Pi}^{\perp} D \phi
$$

so the related delta functional becomes

$$
\operatorname{Det}\left[-\vec{\nabla}^{2}\right] \delta\left[\vec{\nabla} \cdot \vec{\Pi}-g \bar{\psi} \gamma^{0} \psi\right] \longrightarrow \operatorname{Det}\left[-\vec{\nabla}^{2}\right] \delta\left[-\vec{\nabla}^{2} \phi-g \bar{\psi} \gamma^{0} \psi\right]=\delta\left[\phi-\frac{-1}{\vec{\nabla}^{2}}\left(g \bar{\psi} \gamma^{0} \psi\right)\right]
$$

Performing all the delta functional integration, one obtains 


$$
Z=\int D \bar{\psi} D \psi D \overrightarrow{\Pi^{\perp}} D \overrightarrow{A^{\perp}} e^{i \int \bar{\psi} i \gamma^{0} \partial_{t} \psi+\overrightarrow{\Pi^{\perp}} \cdot\left[-\partial_{t} \overrightarrow{A^{\perp}}\right]-\mathcal{H}_{\text {Coulomb }}}
$$

with

$$
\mathcal{H}_{\text {Coulomb }}=\bar{\psi}(-i \vec{\gamma} \cdot \vec{\nabla}+m) \psi-g \bar{\psi} \vec{\gamma} \psi \cdot \overrightarrow{A^{\perp}}+\frac{1}{2}{\overrightarrow{\Pi^{\perp}}}^{2}+\frac{1}{2} \vec{B}^{2}+\frac{1}{2} g^{2}\left(\bar{\psi} \gamma^{0} \psi\right) \frac{-1}{\vec{\nabla}^{2}}\left(\bar{\psi} \gamma^{0} \psi\right) .
$$

Equivalently, in terms of Lagrangian:

$$
Z=\int D \bar{\psi} D \psi D \overrightarrow{A^{\perp}} e^{i \int \mathcal{L}_{\text {Coulomb }}}
$$

where

$$
\mathcal{L}_{\text {Coulomb }}=\bar{\psi}(i \not \supset-m) \psi+g \bar{\psi} \vec{\gamma} \psi \cdot \overrightarrow{A^{\perp}}+\frac{1}{2}\left(\partial_{t}{\overrightarrow{A^{\perp}}}^{2}\right)^{2}-\frac{1}{2}\left(\vec{\nabla} \times \overrightarrow{A^{\perp}}\right)^{2}-\frac{1}{2} g^{2}\left(\bar{\psi} \gamma^{0} \psi\right) \frac{-1}{\vec{\nabla}^{2}}\left(\bar{\psi} \gamma^{0} \psi\right) .
$$

Here, we have $\bar{\psi}, \psi$ and $\overrightarrow{A^{\perp}}$ as the fields degrees of freedom. Note that $\frac{-1}{\vec{\nabla}^{2}}$ has the spatial representation $\frac{1}{4 \pi\left|\vec{x}-\overrightarrow{x^{\prime}}\right|}$.

As advertised, the Coulomb potential makes its appearance explicitly in the Lagrangian, and hence will appear in any tree level calculations. 


\section{B.3.2 COULOMB GAUGE IN QCD}

We now turn to the discussion of Coulomb gauge fixing in QCD $[22,62]$. The non-abelian nature of the theory introduces several complications. Firstly, the Faddeev-Popov determinant is no longer field independent. In other gauge, this is usually handled by the introduction of auxiliary ghost fields. As we shall see, being a physical gauge, there is no need to introduce the ghost degrees of freedom in Coulomb gauge. Again, one integrates away $A^{0}$ and obtains a generalized Coulomb potential in the Lagrangian. We will also discuss the Gribov problem, which originates from the inability of the gauge condition to fix the gauge uniquely.

The Lagrangian density of QCD reads

$$
\mathcal{L}_{Q C D}=\bar{\psi}(i \not \supset-M-g \not) \psi-\frac{1}{4} G^{2}
$$

with

$$
\begin{aligned}
{\left[T^{a}, T^{b}\right] } & =i f^{a b c} T^{c} \\
A & =T^{a} A^{a} \\
G_{\mu \nu}^{a} & =\partial_{\mu} A_{\nu}^{a}-\partial_{\nu} A_{\mu}^{a}-g f^{a b c} A_{\mu}^{b} A_{\nu}^{c} .
\end{aligned}
$$

The Lagrangian is invariant under the gauge transformation:

$$
\begin{aligned}
& \psi \longrightarrow e^{-i g \alpha^{a} T^{a}} \psi \\
& A_{\mu}^{a} \longrightarrow A_{\mu}^{a \prime} \approx A_{\mu}^{a}+\partial_{\mu} \alpha^{a}-g f^{a b c} A_{\mu}^{b} \alpha^{c}
\end{aligned}
$$

It is useful to define the operator

$$
\mathcal{D}_{\mu}^{a c}=\partial_{\mu} \delta^{a c}-g f^{a b c} A_{\mu}^{b},
$$

so that the gauge transformation for photon fields can be conveniently written as 


$$
A_{\mu}^{a \prime} \approx A_{\mu}^{a}+\mathcal{D}_{\mu}^{a c} \alpha_{c}
$$

Again, we define the electric and magnetic fields:

$$
\begin{aligned}
E_{a}^{i} & =G_{a}^{i 0}=\partial^{i} A_{a}^{0}-\partial_{t} A_{a}^{i}-g f_{a b c} A_{b}^{i} A_{c}^{0} \\
B^{i} & =-\frac{1}{2} \epsilon^{i j k} G_{j k}
\end{aligned}
$$

Parallel to the discussion in QED, we note

$$
e^{i \int \frac{1}{2} G_{a}^{i 0} G_{a}^{i 0}}=\int D \vec{\Pi} e^{i \int-\frac{1}{2} \Pi^{i} \Pi^{i}+\Pi^{i} G_{a}^{i 0}}
$$

the generating functional is rewritten as

$$
\begin{aligned}
Z=\int & D \bar{\psi} D \psi D \vec{A} D A^{0} D \vec{\Pi} \delta[\vec{\nabla} \cdot \vec{A}] \operatorname{Det}[-\vec{\nabla} \cdot \overrightarrow{\mathcal{D}}] \\
& e^{i \int \bar{\psi}(i \not \supset-m) \psi+g \bar{\psi} \vec{\gamma} T^{a} \psi \cdot \vec{A}^{a}+\vec{\Pi}^{a} \cdot\left[\partial_{t} \overrightarrow{A^{a}}\right]-\frac{1}{2} \vec{B}^{2}-\frac{1}{2} \vec{\Pi}^{2}+\left[-g \bar{\psi} \gamma^{0} T^{a} \psi+\Pi_{a}^{i} \partial^{i}+g f_{a b c} A_{b}^{i} \Pi_{c}^{i}\right] A_{a}^{0}} .
\end{aligned}
$$

Integrating out $A^{0}$

$$
\int D A^{0} e^{i \int\left[-g \bar{\psi} \gamma^{0} T^{a} \psi+\Pi_{a}^{i} \partial^{i}+g f_{a b c} A_{b}^{i} \Pi_{c}^{i}\right] A^{0}}=\delta\left[\overrightarrow{\mathcal{D}} \cdot \vec{\Pi}-g \bar{\psi} \gamma^{0} T^{a} \psi\right]
$$

Rewriting $\vec{\Pi}$ as

$$
\begin{aligned}
\vec{\Pi} & =-\vec{\nabla} \phi+\overrightarrow{\Pi^{\perp}} \\
\vec{\nabla} \cdot \overrightarrow{\Pi^{\perp}} & =0 \\
\frac{1}{2} \vec{\Pi}^{2} & =\frac{1}{2}{\overrightarrow{\Pi^{\perp}}}^{2}+\frac{1}{2} \phi\left(-\vec{\nabla}^{2}\right) \phi
\end{aligned}
$$

The functional integral of $\vec{\Pi}$ can be decomposed into 


$$
D \vec{\Pi} \longrightarrow D \overrightarrow{\Pi^{\perp}} D \phi .
$$

The related delta functional reads

$$
\operatorname{Det}[-\vec{\nabla} \cdot \mathcal{D}] \delta\left[\overrightarrow{\mathcal{D}} \cdot \Pi-g \bar{\psi} \gamma^{0} T^{a} \psi\right] \longrightarrow \operatorname{Det}[-\vec{\nabla} \cdot \mathcal{D}] \delta\left[-\overrightarrow{\mathcal{D}} \cdot \vec{\nabla} \phi-\left(g \bar{\psi} \gamma^{0} T^{a} \psi+g f_{a b c} A_{i}^{b} \Pi_{c}^{\perp^{i}}\right)\right]
$$

We identify the non-abelian version of the density of the source

$$
\rho_{\text {tot }}^{a}=g \bar{\psi} \gamma^{0} T^{a} \psi+g f_{a b c} A_{i}^{b} \Pi_{c}^{\perp^{i}}
$$

hence the delta functional becomes (within Coulomb gauge)

$$
\operatorname{Det}[-\vec{\nabla} \cdot \mathcal{D}] \delta\left[-\overrightarrow{\mathcal{D}} \cdot \vec{\nabla} \phi-\rho_{t o t}\right]=\delta\left[\phi-\frac{-1}{\vec{\nabla} \cdot \mathcal{D}} \rho_{t o t}\right]
$$

Finally, we reach

$$
Z=\int D \bar{\psi} D \psi D \overrightarrow{\Pi^{\perp}} D \overrightarrow{A^{\perp}} e^{i \int \bar{\psi} i \gamma^{0} \partial_{t} \psi+\Pi_{a}^{\vec{\perp}} \cdot\left[-\partial_{t} \overrightarrow{A_{a}^{\perp}}\right]-\mathcal{H}_{\text {Coulomb }}}
$$

with

$$
\begin{aligned}
\mathcal{H}_{\text {Coulomb }} & =\frac{1}{2}{\overrightarrow{\Pi^{\perp}}}^{2}+\frac{1}{2} \vec{B}^{2}+\bar{\psi}(-i \vec{\gamma} \cdot \vec{\nabla}+m) \psi-g \bar{\psi} \vec{\gamma} \psi \cdot \overrightarrow{A^{\perp}}+\frac{1}{2} g^{2} \rho_{\text {tot }} \frac{1}{\vec{\nabla} \cdot \overrightarrow{\mathcal{D}}}\left(-\vec{\nabla}^{2}\right) \frac{1}{\vec{\nabla} \cdot \overrightarrow{\mathcal{D}}} \rho_{\text {tot }} \\
\rho_{\text {tot }}^{a} & =g \bar{\psi} \gamma^{0} T^{a} \psi+g f_{a b c} A_{i}^{b} \Pi_{c}^{\perp^{i}} .
\end{aligned}
$$

The non-abelian Coulomb gauge condition introduces a generalized Coulomb potential in the Hamiltonian level.

Lastly we turn to a brief discussion of Gribov problem in the context of coulomb gauge [63]. 
For the gauge condition to select the representative uniquely, one commands that the solution for

$$
\vec{\nabla} \cdot \overrightarrow{A^{\alpha}}=0
$$

to be unique.

In $\mathrm{QED}$, it presents no problem as the solution to $-\vec{\nabla}^{2} \alpha=0$ is unique for a given boundary condition.

For the non-abelian case, however, one has

$$
\begin{aligned}
\left(-\vec{\nabla} \cdot \overrightarrow{\mathcal{D}}\left[\overrightarrow{A^{\perp}}\right]\right) \alpha & =0 \\
-\vec{\nabla}^{2} \alpha^{a}-g f_{a b c} \overrightarrow{A^{b}} \cdot \vec{\nabla} \alpha^{c} & =0 .
\end{aligned}
$$

Gribov pointed out the equation admits non-trivial solutions in this case. A resolution suggested by Zwanziger[64] is to restrict the configuration space to the fundamental modular region, which consists of the global minimum of the functional

$$
\int \operatorname{Tr}\left[\overrightarrow{A^{\alpha}} \cdot \overrightarrow{A^{\alpha}}\right]
$$

along a given gauge orbit.

The study of the operator $-\vec{\nabla} \cdot \overrightarrow{\mathcal{D}}$ in Gribov problem is deeply related to the color confinement problem. The Gribov confinement scenario states that the Coulomb potential $\frac{1}{\vec{\nabla} \cdot \overrightarrow{\mathcal{D}}}\left(-\vec{\nabla}^{2}\right) \frac{1}{\vec{\nabla} \cdot \overrightarrow{\mathcal{D}}}$ is long range due to the fact that $\frac{1}{\vec{\nabla} \cdot \overrightarrow{\mathcal{D}}}$ is infrared-enhanced. At large quark separation, the configuration corresponds to zero-eigenvalue mode of $-\vec{\nabla} \cdot \overrightarrow{\mathcal{D}}$ dominates, such configurations are believed to be located near the boundary of fundamental modular region and are related to center vortexes picture of the confinement [65, 66, 23].

This concludes our review on functional methods in QFT. 


\section{APPENDIX C}

\section{NOTES ON PERFORMING MATSUBARA SUM}

We will illustrate the techniques of Matsubara sum through the computation of propagators:

Firstly for bosonic propagator:

$$
G_{k^{0}, \vec{k}}=\frac{1}{k^{0^{2}}-\epsilon_{\vec{k}}^{2}+i \epsilon}
$$

and hence

$$
\begin{aligned}
\int \frac{d k^{0}}{2 \pi} e^{-i k^{0} t} G_{k^{0} \vec{k}} & =\frac{-i}{2 \epsilon_{\vec{k}}} e^{-i \epsilon_{\vec{k}} t} & & t>0 \\
& =\frac{-i}{2 \epsilon_{\vec{k}}} e^{+i \epsilon_{\vec{k}} t} & & t<0
\end{aligned}
$$

Consider

$$
G_{\omega_{n}, \vec{k}}=\frac{1}{\left(i \omega_{n}+\mu\right)^{2}-\epsilon_{\vec{k}}^{2}}
$$

and we are interested in the Matsubara sum:

$$
G_{\vec{k}}[\tau]=\frac{1}{\beta} \sum_{\omega_{n}} \frac{1}{\left(i \omega_{n}+\mu\right)^{2}-\epsilon_{\vec{k}}^{2}} e^{-i \omega_{n} \tau}
$$

where $\omega_{n}=\frac{2 n \pi}{\beta}$ 
We will evaluate the Matsubara sum using the method of pole generation:

The method of pole generation is a trick that helps to evaluate the sum by considering an integral.

consider

$$
\mathcal{I}=\oint \frac{d z}{2 \pi i} n(z) \frac{1}{(z+\mu)^{2}+\epsilon_{\vec{k}}^{2}} e^{-z \tau}
$$

where $n(z)$ has poles at $i \omega_{n}$

The choice of this $n(z)$ is not unique, and must be chosen such that the integral $\mathcal{I}$ gives 0.

If $\tau>0$.

we need to pick:

$$
n(z)=\frac{-1}{e^{-\beta z}-1}
$$

so that the integral $\mathcal{I}$ is zero.

Observing the fact that the integral is given by its residues, we have

$$
0=\mathcal{I}=\frac{-1}{\left.e^{\beta\left(-\epsilon_{\vec{k}}-\mu\right.}\right)-1}\left(\frac{1}{2 \epsilon_{\vec{k}}}\right) e^{-\left(\epsilon_{\vec{k}}-\mu\right) \tau}+\frac{-1}{e^{\beta\left(\epsilon_{\vec{k}}+\mu\right)}-1}\left(\frac{1}{2 \epsilon_{\vec{k}}}\right) e^{\left(\epsilon_{\vec{k}}+\mu\right) \tau}+\sum_{\omega_{n}} \frac{1}{\left(i \omega_{n}+\mu\right)^{2}-\epsilon_{\vec{k}}^{2}}
$$

giving

$$
\sum_{\omega_{n}} \frac{1}{\left(i \omega_{n}+\mu\right)^{2}-\epsilon_{\vec{k}}^{2}} e^{-i \omega_{n} \tau}=\frac{-1}{2 \epsilon_{\vec{k}}}\left[\left(n_{\vec{k}}+1\right) e^{-\left(\epsilon_{\vec{k}}-\mu\right) \tau}+\bar{n}_{\vec{k}} e^{\left(\epsilon_{\vec{k}}+\mu\right) \tau}\right]
$$

with

$$
\begin{aligned}
& n_{\vec{k}}=\frac{1}{e^{\beta\left(E_{\vec{k}}-\mu\right)}-1} \\
& \bar{n}_{\vec{k}}=\frac{1}{e^{\beta\left(E_{\vec{k}}+\mu\right)}-1}
\end{aligned}
$$

On the other hand, if $\tau<0$.

we need to pick $n(z)=\frac{1}{e^{\beta z}-1}$ and the corresponding Matsubara sum is given by 


$$
\sum_{\text {other poles }} \operatorname{Res}\left[n(z) \frac{1}{(z+\mu)^{2}+\epsilon_{\vec{k}}^{2}} e^{-z \tau}\right]
$$

which gives

$$
\sum_{\omega_{n}} \frac{1}{\left(i \omega_{n}+\mu\right)^{2}-\epsilon_{\vec{k}}^{2}} e^{-i \omega_{n} \tau}=\frac{-1}{2 \epsilon_{\vec{k}}}\left[n_{\vec{k}} e^{-\left(\epsilon_{\vec{k}}-\mu\right) \tau}+\left(1+\bar{n}_{\vec{k}}\right) e^{\left(\epsilon_{\vec{k}}+\mu\right) \tau}\right]
$$

The $\tau \rightarrow 0$. limit of the sum is well defined, as we can clearly seen above, however, it is a useful practice to consider

$$
\sum_{\omega_{n}} \frac{1}{\left(i \omega_{n}+\mu\right)^{2}-\epsilon_{\vec{k}}^{2}}
$$

by itself.

In this case, we pick $n(z)=\frac{1}{2} \operatorname{coth}\left[\frac{1}{2} \beta z\right]$, which is bounded everywhere,

$$
\sum_{\omega_{n}} \frac{1}{\left(i \omega_{n}+\mu\right)^{2}-\epsilon_{\vec{k}}^{2}}=\frac{-1}{2 \epsilon_{\vec{k}}}\left[1+n_{\vec{k}}+\bar{n}_{\vec{k}}\right]
$$

It is also essential to observe that at $T, \mu \rightarrow 0$, we can identify

$$
G_{Q F T}(t)=i \lim _{T, \mu \rightarrow 0 .} G_{F T}(\tau=i t)
$$

The three pole generators will give different results if the sum is not well defined. For example, consider the sum

$$
\sum_{\omega_{n}} \frac{\omega_{n}}{\left(\omega_{n}\right)^{2}+\epsilon_{\vec{k}}^{2}}
$$

The sum can be obtained by $\left.i \frac{d}{d \tau} G\right|_{\tau \rightarrow 0^{+}}$or $\left.i \frac{d}{d \tau} G\right|_{\tau \rightarrow 0^{-}}$, the two sums are different, while the third pole generator gives zero, which is the average of the above two. This comes from the fact that the sum is not well defined, as $\frac{d}{d \tau} G$ is not a well defined function at $\tau \rightarrow 0$. However an intuitive answer for the above sum is 0 , and the third pole generator gives a sensible answer by computing the average of the relevant function: 


$$
\frac{1}{2}\left[f\left(\tau \rightarrow 0^{+}\right)+f\left(\tau \rightarrow 0^{-}\right)\right]
$$

The corresponding sum for fermionic frequencies can be constructed similarly, here we list the various useful result for reference:

- bosonic propagator:

$$
\begin{aligned}
& G_{k^{0}, \vec{k}}=\frac{1}{k^{0}-\epsilon_{\vec{k}}^{2}+i \epsilon} \\
& \int \frac{d k^{0}}{2 \pi} e^{-i k^{0} t} G_{k^{0} \vec{k}}=\frac{-i}{2 \epsilon_{\vec{k}}} e^{-i \epsilon_{\vec{k}} t} \quad t>0 \\
& =\frac{-i}{2 \epsilon_{\vec{k}}} e^{+i \epsilon_{\vec{k}} t} \quad t<0 \\
& G_{\omega_{n}, \vec{k}}=\frac{1}{\left(i \omega_{n}+\mu\right)^{2}-\epsilon_{\vec{k}}^{2}} \\
& \sum_{\omega_{n}} \frac{1}{\left(i \omega_{n}+\mu\right)^{2}-\epsilon_{\vec{k}}^{2}} e^{-i \omega_{n} \tau}=\frac{-1}{2 \epsilon_{\vec{k}}}\left[\left(n_{\vec{k}}+1\right) e^{-\left(\epsilon_{\vec{k}}-\mu\right) \tau}+\bar{n}_{\vec{k}} e^{\left(\epsilon_{\vec{k}}+\mu\right) \tau}\right] \quad \tau>0 \\
& =\frac{-1}{2 \epsilon_{\vec{k}}}\left[n_{\vec{k}} e^{-\left(\epsilon_{\vec{k}}-\mu\right) \tau}+\left(\bar{n}_{\vec{k}}+1\right) e^{\left(\epsilon_{\vec{k}}+\mu\right) \tau}\right] \quad \tau<0 \\
& =\frac{-1}{2 \epsilon_{\vec{k}}}\left[1+n_{\vec{k}}+\bar{n}_{\vec{k}}\right] \quad \tau=0
\end{aligned}
$$

with pole generator

$$
\begin{aligned}
n(z)= & \frac{-1}{e^{-\beta z}-1} & & \tau>0 \\
& =\frac{1}{e^{\beta z}-1} & & \tau<0 \\
& =\frac{1}{2} \operatorname{coth}\left[\frac{1}{2} \beta z\right] & & \tau=0
\end{aligned}
$$


- fermionic propagator

$$
S_{k^{0}, \vec{k}}=\frac{1}{\not k-M+i \epsilon}
$$

it is useful to define:

$$
\begin{aligned}
\sum u_{\vec{k}} \bar{u}_{\vec{k}} & =\frac{\epsilon_{\vec{k}} \gamma^{0}-\vec{k} \cdot \vec{\gamma}+M}{2 \epsilon_{\vec{k}}} \\
\sum v_{-\vec{k}} \bar{v}_{-\vec{k}} & =\frac{\epsilon_{\vec{k}} \gamma^{0}+\vec{k} \cdot \vec{\gamma}-M}{2 \epsilon_{\vec{k}}}
\end{aligned}
$$

then

$$
\begin{array}{cc}
\int \frac{d k^{0}}{2 \pi} e^{-i k^{0} t} S_{k^{0} \vec{k}}=-i\left(\sum u_{\vec{k}} \bar{u}_{\vec{k}}\right) e^{-i \epsilon_{\vec{k}} t} & t>0 \\
=-i(-1)\left(\sum v_{-\vec{k}} \bar{v}_{-\vec{k}}\right) e^{i \epsilon_{\vec{k}} t} & t<0 \\
S_{\omega_{n}, \vec{k}}=\frac{1}{\left(i \omega_{n}+\mu\right) \gamma^{0}-\vec{k} \cdot \vec{\gamma}-M} & \\
\sum_{\omega_{n}} \frac{1}{\left(i \omega_{n}+\mu\right) \gamma^{0}-\vec{k} \cdot \vec{\gamma}-M} e^{-i \omega_{n} \tau} & \tau>0 \\
=(-1)\left[\left(1-n_{\vec{k}}\right) e^{-\left(\epsilon_{\vec{k}}-\mu\right) \tau} \sum u_{\vec{k}} \bar{u}_{\vec{k}}+\bar{n}_{\vec{k}} e^{\left(\epsilon_{\vec{k}}+\mu\right) \tau} \sum v_{-\vec{k}} \bar{v}_{-\vec{k}}\right] & \tau<0 \\
=(+1)\left[n_{\vec{k}} e^{-\left(\epsilon_{\vec{k}}-\mu\right) \tau} \sum u_{\vec{k}} \bar{u}_{\vec{k}}+\left(1-\bar{n}_{\vec{k}}\right) e^{\left(\epsilon_{\vec{k}}+\mu\right) \tau} \sum v_{-\vec{k}} \bar{v}_{-\vec{k}}\right] &
\end{array}
$$

with pole generator

$$
\begin{aligned}
n(z) & =\frac{1}{e^{-\beta z}+1} & & \tau>0 \\
& =\frac{-1}{e^{\beta z}+1} & & \tau<0 \\
& =\frac{1}{2} \tanh \left[\frac{1}{2} \beta z\right] & & \tau=0
\end{aligned}
$$


note that our key result is:

$$
\sum_{\omega_{n}} \frac{1}{\left(i \omega_{n}+\mu\right) \gamma^{0}-\vec{k} \cdot \vec{\gamma}-M}=\frac{1}{2}\left(n_{\vec{k}}-\bar{n}_{\vec{k}}\right) \gamma^{0}+(-1)\left(1-n_{\vec{k}}-\bar{n}_{\vec{k}}\right) \frac{-\vec{k} \cdot \vec{\gamma}+M}{2 \epsilon_{\vec{k}}}
$$


APPENDIX D

FINAL FORM OF THE GAP EQUATIONS FOR QED3 AT $T=0$

We record the final form of the gap equations for QED3 in 2-spinor formalism at $T=0$.

\section{D.1 BALL-CHU VERTEX}

- formula for $B[p]$ :

$$
\begin{gathered}
B_{p}=m-i g^{2} \int \frac{d^{3} l}{(2 \pi)^{3}} \frac{-1}{A_{l}^{2} l^{2}-B_{l}^{2}}\left[\tilde{\Omega}_{B 1}+\tilde{\Omega}_{B 2}+\tilde{\Omega}_{B 3}\right] \\
\tilde{\Omega}_{B 1}=\tilde{\Omega}_{B 1 \text { sym }}+\tilde{\Omega}_{B 1 \text { anti-sym }} \\
\tilde{\Omega}_{B 1 \text { sym }}=\Omega_{1} B_{l}\left[\frac{-2}{\left[k^{2}+F\right]-\frac{\left(\mu_{C S}-\mathcal{F}\right)^{2} k^{2}}{\left[k^{2}+F\right]}}-\frac{\xi}{k^{2}}\right] \\
\tilde{\Omega}_{B 1 \text { anti-sym }}=\frac{-2 \Omega_{1} A_{l}}{\left[k^{2}+F\right]-\frac{\left(\mu_{C S-\mathcal{F})^{2} k^{2}}\right.}{\left[k^{2}+F\right]}}
\end{gathered}
$$




$$
\tilde{\Omega}_{B 2}=\tilde{\Omega}_{B 2 s y m}+\tilde{\Omega}_{B 2 a n t i-s y m}
$$

$$
\tilde{\Omega}_{B 2 s y m}=\Omega_{2} B_{l}\left[\frac{-R^{2}+(k \cdot R)^{2} \frac{1}{k^{2}}}{\left[k^{2}+F\right]-\frac{\left(\mu_{C S}-\mathcal{F}\right)^{2} k^{2}}{\left[k^{2}+F\right]}}-\frac{\xi}{k^{2}} \frac{(k \cdot R)^{2}}{k^{2}}\right]
$$

$$
\tilde{\Omega}_{B 2 a n t i-s y m}=\frac{\Omega_{2} A_{l}}{\left[k^{2}+F\right]-\frac{\left(\mu_{C S}-\mathcal{F}\right)^{2} k^{2}}{\left[k^{2}+F\right]}} \frac{\left(\mu_{C S}-\mathcal{F}\right)}{k^{2}+F}\left[(R \cdot k)(R \cdot l)-R^{2}(l \cdot k)\right]
$$

$$
\tilde{\Omega}_{B 3}=\Omega_{3} A_{l}\left[\frac{-l \cdot R+\frac{(l \cdot k)(k \cdot R)}{k^{2}}}{\left[k^{2}+F\right]-\frac{\left(\mu_{C S} \mathcal{F}\right)^{2} k^{2}}{\left[k^{2}+F\right]}}-\frac{\xi}{k^{2}} \frac{(l \cdot k)(k \cdot R)}{k^{2}}\right]
$$

$$
\begin{aligned}
R^{\mu} & =l^{\mu}+p^{\mu} \\
k^{\mu} & =p^{\mu}-l^{\mu} \\
\Omega_{1} & =\frac{A_{p}+A_{l}}{2} \\
\Omega_{2} & =\frac{1}{p^{2}-l^{2}} \frac{A_{p}-A_{l}}{2} \\
\Omega_{3} & =-\frac{1}{p^{2}-l^{2}}\left(B_{p}-B_{l}\right)
\end{aligned}
$$


- formula for $A[p]$ :

$$
\begin{aligned}
& A_{p}=1-i g^{2} \int \frac{d^{3} l}{(2 \pi)^{3}} \frac{-1}{A_{l}^{2} l^{2}-B_{l}^{2}} \frac{-1}{p^{2}}\left[\tilde{\Omega}_{A 1}+\tilde{\Omega}_{A 2}+\tilde{\Omega}_{A 3}\right] \\
& \tilde{\Omega}_{A 1}=\tilde{\Omega}_{A 1 s y m}+\tilde{\Omega}_{A 1 \text { anti-sym }} \\
& \tilde{\Omega}_{A 1 s y m}=\Omega_{1} A_{l}\left[\frac{2 \frac{p \cdot k l \cdot k}{k^{2}}}{\left[k^{2}+F\right]-\frac{\left(\mu_{C S}-\mathcal{F}\right)^{2} k^{2}}{\left[k^{2}+F\right]}}-\frac{\xi}{k^{2}}\left[\frac{2 p \cdot k l \cdot k}{k^{2}}-p \cdot l\right]\right] \\
& \tilde{\Omega}_{\text {A1anti-sym }}=\frac{2 \Omega_{1} B_{l}}{\left[k^{2}+F\right]-\frac{\left(\mu_{C S}-\mathcal{F}\right)^{2} k^{2}}{\left[k^{2}+F\right]}} \frac{(p \cdot k)\left(\mu_{C S}-\mathcal{F}\right)}{k^{2}+F} \\
& \tilde{\Omega}_{A 2}=\tilde{\Omega}_{A 2 s y m}+\tilde{\Omega}_{A 2 a n t i-s y m} \\
& \tilde{\Omega}_{A 2 s y m}=\Omega_{2} A_{l}\left[\frac{-2(p \cdot R)(l \cdot R)+R^{2}(p \cdot l)+(k \cdot R) \frac{[(p \cdot k)(l \cdot R)+(l \cdot k)(p \cdot R)-(R \cdot k)(p \cdot l)]}{k^{2}}}{\left[k^{2}+F\right]-\frac{\left(\mu_{C S}-\mathcal{F}\right)^{2} k^{2}}{\left[k^{2}+F\right]}}-\right. \\
& \left.\frac{\xi}{k^{2}} \frac{(R \cdot k)[(p \cdot k)(l \cdot R)+(l \cdot k)(p \cdot R)-(R \cdot k)(p \cdot l)]}{k^{2}}\right] \\
& \tilde{\Omega}_{A 2 a n t i-s y m}=\frac{\Omega_{2} B_{l}}{\left[k^{2}+F\right]-\frac{\left(\mu_{C S}-\mathcal{F}\right)^{2} k^{2}}{\left[k^{2}+F\right]}} \frac{\left(\mu_{C S}-\mathcal{F}\right)}{k^{2}+F}\left[(p \cdot k) R^{2}-(p \cdot R)(k \cdot R)\right] \\
& \tilde{\Omega}_{A 3}=\tilde{\Omega}_{A 3 s y m}+\tilde{\Omega}_{A 3 a n t i-s y m} \\
& \tilde{\Omega}_{A 3 s y m}=\Omega_{3} B_{l}\left[\frac{-p \cdot R+\frac{(p \cdot k)(k \cdot R)}{k^{2}}}{\left[k^{2}+F\right]-\frac{\left(\mu_{C S}-\mathcal{F}\right)^{2} k^{2}}{\left[k^{2}+F\right]}}-\frac{\xi}{k^{2}} \frac{p \cdot k k \cdot R}{k^{2}}\right]
\end{aligned}
$$




$$
\tilde{\Omega}_{A 3 a n t i-s y m}=\frac{\Omega_{3} A_{l}}{\left[k^{2}+F\right]-\frac{\left(\mu_{C S}-\mathcal{F}\right)^{2} k^{2}}{\left[k^{2}+F\right]}} \frac{\left(\mu_{C S}-\mathcal{F}\right)}{k^{2}+F}[(p \cdot k)(R \cdot l)-(p \cdot R)(l \cdot k)]
$$

$$
\begin{aligned}
R^{\mu} & =l^{\mu}+p^{\mu} \\
k^{\mu} & =p^{\mu}-l^{\mu} \\
\Omega_{1} & =\frac{A_{p}+A_{l}}{2} \\
\Omega_{2} & =\frac{1}{p^{2}-l^{2}} \frac{A_{p}-A_{l}}{2} \\
\Omega_{3} & =-\frac{1}{p^{2}-l^{2}}\left(B_{p}-B_{l}\right)
\end{aligned}
$$

- formula for $F[k]$ :

$$
\begin{gathered}
F[k]=-i \frac{1}{2} g^{2} \int \frac{d^{3} l}{(2 \pi)^{3}} \frac{1}{A_{1}^{2} l_{1}^{2}-B_{1}^{2}} \frac{1}{A_{2}^{2} l_{2}^{2}-B_{2}^{2}}(\operatorname{tr} I)\left[\tilde{\Omega}_{F 1}+\tilde{\Omega}_{F 2}+\tilde{\Omega}_{F 3}\right] \\
\tilde{\Omega}_{F 1}=\Omega_{1} 2 A_{1} A_{2}\left[l_{1} \cdot l_{2}-3 \frac{\left(k \cdot l_{1}\right)\left(k \cdot l_{2}\right)}{k^{2}}\right] \\
\left.+B_{1} B_{2}\left[R^{2}-3 \frac{(k \cdot R)^{2}}{k^{2}}\right]\right] \\
\left.\frac{-3}{k^{2}}(R \cdot k)\left[\left(l_{1} \cdot k\right)\left(l_{2} \cdot R\right)+\left(l_{2} \cdot k\right)\left(l_{1} \cdot R\right)-(R \cdot k)\left(l_{1} \cdot l_{2}\right)\right]\right\} \\
\tilde{\Omega}_{F 3}=\Omega_{3}\left[A_{1} B_{2}\left[\left(l_{1} \cdot R\right)-3 \frac{\left(k \cdot l_{1}\right)(k \cdot R)}{k^{2}}\right]+A_{2} B_{1}\left[\left(l_{2} \cdot R\right)-3 \frac{\left(k \cdot l_{2}\right)(k \cdot R)}{k^{2}}\right]\right]
\end{gathered}
$$




$$
\begin{aligned}
& R^{\mu}=2 l^{\mu}-k^{\mu} \\
& l_{1}{ }^{\mu}=l^{\mu} \\
& l_{2}{ }^{\mu}=l^{\mu}-k^{\mu} \\
& \Omega_{1}=\frac{A_{2}+A_{1}}{2} \\
& \Omega_{2}=\frac{1}{l_{2}^{2}-l_{1}^{2}} \frac{A_{2}-A_{1}}{2} \\
& \Omega_{3}=-\frac{1}{l_{2}^{2}-l_{1}^{2}}\left(B_{2}-B_{1}\right)
\end{aligned}
$$

- formula for $\mathcal{F}[k]$ :

$$
\begin{gathered}
\mathcal{F}=i \frac{1}{2 k^{2}} g^{2} \int \frac{d^{3} l}{(2 \pi)^{3}} \frac{1}{A_{1}^{2} l_{1}^{2}-B_{1}^{2}} \frac{1}{A_{2}^{2} l_{2}^{2}-B_{2}^{2}}(\operatorname{tr} I)\left[\tilde{\Omega}_{\mathcal{F} 1}+\tilde{\Omega}_{\mathcal{F} 2}+\tilde{\Omega}_{\mathcal{F} 3}\right] \\
\tilde{\Omega}_{\mathcal{F} 1}=2 \Omega_{1}\left[A_{1} B_{2} l_{1} \cdot k-A_{2} B_{1} l_{2} \cdot k\right] \\
\tilde{\Omega}_{\mathcal{F} 2}=\Omega_{2}\left\{A_{1} B_{2}\left[R^{2}\left(k \cdot l_{1}\right)-\left(R \cdot l_{1}\right)(R \cdot k)\right]-A_{2} B_{1}\left[R^{2}\left(k \cdot l_{2}\right)-\left(R \cdot l_{2}\right)(R \cdot k)\right]\right\} \\
\tilde{\Omega}_{\mathcal{F} 3}=\Omega_{3} A_{1} A_{2}\left[\left(k \cdot l_{1}\right)\left(R \cdot l_{2}\right)-\left(k \cdot l_{2}\right)\left(R \cdot l_{1}\right)\right] \\
R^{\mu}=2 l^{\mu}-k^{\mu} \\
l_{1}{ }^{\mu}=l^{\mu} \\
l_{2}{ }^{\mu}=l^{\mu}-k^{\mu} \\
\Omega_{1}=\frac{A_{2}+A_{1}}{2} \\
\Omega_{2}=\frac{1}{l_{2}^{2}-l_{1}^{2}} \frac{A_{2}-A_{1}}{2} \\
\Omega_{3}=-\frac{1}{l_{2}^{2}-l_{1}^{2}}\left(B_{2}-B_{1}\right) \\
\end{gathered}
$$




\section{D.2 CURTIS-PENNINGTON VERTEX}

The Ball-Chu vertex only specify the longitudinal part of a vertex. The CP truncation scheme is defined as:

$$
\Gamma_{C . P}^{\nu}[p, q]=\Gamma_{B . C}^{\nu}[p, q]+\frac{A_{q}-A_{p}}{2} \frac{q^{2}+p^{2}}{\left(q^{2}-p^{2}\right)^{2}+\left[M_{q}^{2}+M_{p}^{2}\right]^{2}}\left[\left(q^{2}-p^{2}\right) \gamma^{\nu}-(\not q-\not p)(q+p)^{\nu}\right]
$$

It is useful to define:

$$
\begin{gathered}
\chi_{1}=\frac{A_{q}-A_{p}}{2} \frac{q^{4}-p^{4}}{\left(q^{2}-p^{2}\right)^{2}+\left[M_{q}^{2}+M_{p}^{2}\right]^{2}} \\
\chi_{2}=-\frac{A_{q}-A_{p}}{2} \frac{q^{2}+p^{2}}{\left(q^{2}-p^{2}\right)^{2}+\left[M_{q}^{2}+M_{p}^{2}\right]^{2}} \\
R^{\nu}=(q+p)^{\nu} \\
L^{\nu}=(q-p)^{\nu}
\end{gathered}
$$

such that the $\mathrm{CP}$ vertex reads:

$$
\Gamma_{C . P}^{\nu}[p, q]=\Omega_{1}^{\prime} \gamma^{\nu}+\left[\Omega_{2} R^{\nu} R_{\rho} \gamma^{\rho}+\chi_{2} L_{\rho} \gamma^{\rho} R^{\nu}\right]+\Omega_{3} R^{\nu}
$$

with

$$
\Omega_{1}^{\prime}=\Omega_{1}+\chi_{1}
$$




$$
\Omega_{1}=\frac{A_{q}+A_{p}}{2}
$$

$$
\Omega_{2}=\frac{1}{q^{2}-p^{2}} \frac{A_{q}-A_{p}}{2}
$$

$$
\Omega_{3}=-\frac{1}{q^{2}-p^{2}}\left(B_{q}-B_{p}\right)
$$

$$
\begin{gathered}
\chi_{1}=\frac{A_{q}-A_{p}}{2} \frac{q^{4}-p^{4}}{\left(q^{2}-p^{2}\right)^{2}+\left[M_{q}^{2}+M_{p}^{2}\right]^{2}} \\
\chi_{2}=-\frac{A_{q}-A_{p}}{2} \frac{q^{2}+p^{2}}{\left(q^{2}-p^{2}\right)^{2}+\left[M_{q}^{2}+M_{p}^{2}\right]^{2}}
\end{gathered}
$$

$$
\begin{aligned}
R^{\nu} & =(q+p)^{\nu} \\
L^{\nu} & =(q-p)^{\nu} \\
M & =\frac{B}{A}
\end{aligned}
$$

The corresponding gap equations read: 
- formula for $B[p]$ :

$$
\begin{aligned}
& B_{p}=m-i g^{2} \int \frac{d^{3} l}{(2 \pi)^{3}} \frac{-1}{A_{l}^{2} l^{2}-B_{l}^{2}}\left[\tilde{\Omega}_{B 1}+\tilde{\Omega}_{B 2}+\tilde{\chi}_{B 2}+\tilde{\Omega}_{B 3}\right] \\
& \tilde{\Omega}_{B 1}=\tilde{\Omega}_{B 1 s y m}+\tilde{\Omega}_{B 1 \text { anti-sym }} \\
& \tilde{\Omega}_{B 1 s y m}=\Omega_{1}^{\prime} B_{l}\left[\frac{-2}{\left[k^{2}+F\right]-\frac{\left(\mu_{C S}-\mathcal{F}\right)^{2} k^{2}}{\left[k^{2}+F\right]}}-\frac{\xi}{k^{2}}\right] \\
& \tilde{\Omega}_{\text {B1anti-sym }}=\frac{-2 \Omega_{1}^{\prime} A_{l}}{\left[k^{2}+F\right]-\frac{\left(\mu_{C S}-\mathcal{F}\right)^{2} k^{2}}{\left[k^{2}+F\right]}} \frac{(l \cdot k)\left(\mu_{C S}-\mathcal{F}\right)}{k^{2}+F} \\
& \tilde{\Omega}_{B 2}=\tilde{\Omega}_{B 2 s y m}+\tilde{\Omega}_{B 2 a n t i-s y m} \\
& \tilde{\Omega}_{B 2 s y m}=\Omega_{2} B_{l}\left[\frac{-R^{2}+(k \cdot R)^{2} \frac{1}{k^{2}}}{\left[k^{2}+F\right]-\frac{\left(\mu_{C S}-\mathcal{F}\right)^{2} k^{2}}{\left[k^{2}+F\right]}}-\frac{\xi}{k^{2}} \frac{(k \cdot R)^{2}}{k^{2}}\right] \\
& \tilde{\Omega}_{B 2 a n t i-s y m}=\frac{\Omega_{2} A_{l}}{\left[k^{2}+F\right]-\frac{\left(\mu_{C S}-\mathcal{F}\right)^{2} k^{2}}{\left[k^{2}+F\right]}} \frac{\left(\mu_{C S}-\mathcal{F}\right)}{k^{2}+F}\left[(R \cdot k)(R \cdot l)-R^{2}(l \cdot k)\right] \\
& \tilde{\chi}_{B 2}=\tilde{\chi}_{B 2 s y m}+\tilde{\chi}_{B 2 a n t i-s y m} \\
& \tilde{\chi}_{B 2 s y m}=\chi_{2} B_{l}\left[-\frac{\xi}{k^{2}}(R \cdot k)\right]
\end{aligned}
$$




$$
\begin{aligned}
\tilde{\chi}_{B 2 a n t i-s y m}= & \frac{\chi_{2} A_{l}}{\left[k^{2}+F\right]-\frac{\left(\mu_{C S}-\mathcal{F}\right)^{2} k^{2}}{\left[k^{2}+F\right]}} \frac{\left(\mu_{C S}-\mathcal{F}\right)}{k^{2}+F}\left[k^{2}(R \cdot l)-(R \cdot k)(l \cdot k)\right] \\
\tilde{\Omega}_{B 3}= & \Omega_{3} A_{l}\left[\frac{-l \cdot R+\frac{(l \cdot k)(k \cdot R)}{k^{2}}}{\left[k^{2}+F\right]-\frac{\left(\mu_{C S}-\mathcal{F}\right)^{2} k^{2}}{\left[k^{2}+F\right]}}-\frac{\xi}{k^{2}} \frac{(l \cdot k)(k \cdot R)}{k^{2}}\right] \\
R^{\mu} & =l^{\mu}+p^{\mu} \\
k^{\mu} & =p^{\mu}-l^{\mu} \\
\Omega_{1}^{\prime} & =\Omega_{1}+\chi_{1} \\
\Omega_{1} & =\frac{A_{p}+A_{l}}{2} \\
\Omega_{2} & =\frac{1}{p^{2}-l^{2}} \frac{A_{p}-A_{l}}{2} \\
\Omega_{3} & =-\frac{1}{p^{2}-l^{2}}\left(B_{p}-B_{l}\right) \\
\chi_{1} & =\frac{A_{p}-A_{l}}{2} \frac{p^{4}-l^{4}}{\left(p^{2}-l^{2}\right)^{2}+\left[M_{p}^{2}+M_{l}^{2}\right]^{2}} \\
\chi_{2} & =-\frac{A_{p}-A_{l}}{2} \frac{p^{2}+l^{2}}{\left(p^{2}-l^{2}\right)^{2}+\left[M_{p}^{2}+M_{l}^{2}\right]^{2}} \\
M & =\frac{B}{A}
\end{aligned}
$$

- formula for $A[p]$ :

$$
\begin{gathered}
A_{p}=1-i g^{2} \int \frac{d^{3} l}{(2 \pi)^{3}} \frac{-1}{A_{l}^{2} l^{2}-B_{l}^{2}} \frac{-1}{p^{2}}\left[\tilde{\Omega}_{A 1}+\tilde{\Omega}_{A 2}+\tilde{\chi}_{A 2}+\tilde{\Omega}_{A 3}\right] \\
\tilde{\Omega}_{A 1}=\tilde{\Omega}_{A 1 s y m}+\tilde{\Omega}_{A 1 \text { anti-sym }} \\
\tilde{\Omega}_{A 1 s y m}=\Omega_{1}^{\prime} A_{l}\left[\frac{2 \frac{(p \cdot k)(l \cdot k)}{k^{2}}}{\left[k^{2}+F\right]-\frac{\left(\mu_{C S}-\mathcal{F}\right)^{2} k^{2}}{\left[k^{2}+F\right]}}-\frac{\xi}{k^{2}}\left[\frac{2(p \cdot k)(l \cdot k)}{k^{2}}-p \cdot l\right]\right]
\end{gathered}
$$




$$
\begin{aligned}
& \tilde{\Omega}_{\text {A1anti-sym }}=\frac{2 \Omega_{1}^{\prime} B_{l}}{\left[k^{2}+F\right]-\frac{\left(\mu_{C S}-\mathcal{F}\right)^{2} k^{2}}{\left[k^{2}+F\right]}} \frac{(p \cdot k)\left(\mu_{C S}-\mathcal{F}\right)}{k^{2}+F} \\
& \tilde{\Omega}_{A 2}=\tilde{\Omega}_{A 2 s y m}+\tilde{\Omega}_{A 2 a n t i-s y m} \\
& \tilde{\Omega}_{A 2 s y m}=\Omega_{2} A_{l}\left[\frac{-2(p \cdot R)(l \cdot R)+R^{2}(p \cdot l)+(k \cdot R) \frac{[(p \cdot k)(l \cdot R)+(l \cdot k)(p \cdot R)-(R \cdot k)(p \cdot l)]}{k^{2}}}{\left[k^{2}+F\right]-\frac{\left(\mu_{C S}-\mathcal{F}\right)^{2} k^{2}}{\left[k^{2}+F\right]}}-\right. \\
& \left.\frac{\xi}{k^{2}} \frac{(R \cdot k)[(p \cdot k)(l \cdot R)+(l \cdot k)(p \cdot R)-(R \cdot k)(p \cdot l)]}{k^{2}}\right] \\
& \tilde{\Omega}_{A 2 a n t i-s y m}=\frac{\Omega_{2} B_{l}}{\left[k^{2}+F\right]-\frac{\left(\mu_{C S}-\mathcal{F}\right)^{2} k^{2}}{\left[k^{2}+F\right]}} \frac{\left(\mu_{C S}-\mathcal{F}\right)}{k^{2}+F}\left[(p \cdot k) R^{2}-(p \cdot R)(k \cdot R)\right] \\
& \tilde{\chi}_{A 2}=\tilde{\chi}_{A 2 s y m}+\tilde{\chi}_{A 2 a n t i-s y m} \\
& \tilde{\chi}_{A 2 s y m}=\chi_{2} A_{l}\left[\frac{-(R \cdot l)(k \cdot p)-(R \cdot p)(k \cdot l)+\frac{1}{k^{2}} 2(p \cdot k)(l \cdot k)(R \cdot k)}{\left[k^{2}+F\right]-\frac{\left(\mu_{C S}-\mathcal{F}\right)^{2} k^{2}}{\left[k^{2}+F\right]}}-\right. \\
& \left.\frac{\xi}{k^{2}} \frac{1}{k^{2}}\left[\frac{2(p \cdot k)(R \cdot k)(l \cdot k)}{k^{2}}-(p \cdot l)(R \cdot k)\right]\right] \\
& \tilde{\chi}_{A 2 a n t i-s y m}=\frac{\chi_{2} B_{l}}{\left[k^{2}+F\right]-\frac{\left(\mu_{C S}-\mathcal{F}\right)^{2} k^{2}}{\left[k^{2}+F\right]}} \frac{\left(\mu_{C S}-\mathcal{F}\right)}{k^{2}+F}\left[(p \cdot k)(R \cdot k)-(R \cdot p) k^{2}\right] \\
& \tilde{\Omega}_{A 3}=\tilde{\Omega}_{A 3 s y m}+\tilde{\Omega}_{A 3 a n t i-s y m}
\end{aligned}
$$




$$
\begin{gathered}
\tilde{\Omega}_{A 3 s y m}=\Omega_{3} B_{l}\left[\frac{-p \cdot R+\frac{(p \cdot k)(k \cdot R)}{k^{2}}}{\left[k^{2}+F\right]-\frac{\left(\mu_{C S}-\mathcal{F}\right)^{2} k^{2}}{\left[k^{2}+F\right]}}-\frac{\xi}{k^{2}} \frac{(p \cdot k)(k \cdot R)}{k^{2}}\right] \\
\tilde{\Omega}_{A 3 a n t i-s y m}=\frac{\Omega_{3} A_{l}}{\left[k^{2}+F\right]-\frac{\left(\mu_{C S}-\mathcal{F}\right)^{2} k^{2}}{\left[k^{2}+F\right]}} \frac{\left(\mu_{C S}-\mathcal{F}\right)}{k^{2}+F}[(p \cdot k)(R \cdot l)-(p \cdot R)(l \cdot k)]
\end{gathered}
$$

with

$$
\begin{aligned}
R^{\mu} & =l^{\mu}+p^{\mu} \\
k^{\mu} & =p^{\mu}-l^{\mu} \\
\Omega_{1}^{\prime} & =\Omega_{1}+\chi_{1} \\
\Omega_{1} & =\frac{A_{p}+A_{l}}{2} \\
\Omega_{2} & =\frac{1}{p^{2}-l^{2}} \frac{A_{p}-A_{l}}{2} \\
\Omega_{3} & =-\frac{1}{p^{2}-l^{2}}\left(B_{p}-B_{l}\right) \\
\chi_{1} & =\frac{A_{p}-A_{l}}{2} \frac{p^{4}-l^{4}}{\left(p^{2}-l^{2}\right)^{2}+\left[M_{p}^{2}+M_{l}^{2}\right]^{2}} \\
\chi_{2} & =-\frac{A_{p}-A_{l}}{2} \frac{p^{2}+l^{2}}{\left(p^{2}-l^{2}\right)^{2}+\left[M_{p}^{2}+M_{l}^{2}\right]^{2}} \\
M & =\frac{B}{A}
\end{aligned}
$$

- formula for $F[k]$ :

$$
\begin{gathered}
F=-i \frac{1}{2} g^{2} \int \frac{d^{3} l}{(2 \pi)^{3}} \frac{1}{A_{1}^{2} l_{1}^{2}-B_{1}^{2}} \frac{1}{A_{2}^{2} l_{2}^{2}-B_{2}^{2}}(\operatorname{tr} I)\left[\tilde{\Omega}_{F 1}+\tilde{\Omega}_{F 2}+\tilde{\chi}_{F 2}+\tilde{\Omega}_{F 3}\right] \\
\tilde{\Omega}_{F 1}=\Omega_{1}^{\prime} 2 A_{1} A_{2}\left[l_{1} \cdot l_{2}-3 \frac{\left(k \cdot l_{1}\right)\left(k \cdot l_{2}\right)}{k^{2}}\right]
\end{gathered}
$$




$$
\begin{array}{r}
\tilde{\Omega}_{F 2}=\Omega_{2}\left[A _ { 1 } A _ { 2 } \left\{\left[2\left(l_{1} \cdot R\right)\left(l_{2} \cdot R\right)-\left(l_{1} \cdot l_{2}\right) R^{2}\right]+\right.\right. \\
\left.\frac{-3}{k^{2}}(R \cdot k)\left[\left(l_{1} \cdot k\right)\left(l_{2} \cdot R\right)+\left(l_{2} \cdot k\right)\left(l_{1} \cdot R\right)-(R \cdot k)\left(l_{1} \cdot l_{2}\right)\right]\right\} \\
\left.+B_{1} B_{2}\left[R^{2}-3 \frac{(k \cdot R)^{2}}{k^{2}}\right]\right]
\end{array}
$$

$$
\begin{array}{r}
\tilde{\chi}_{F 2}=\chi_{2}\left[A _ { 1 } A _ { 2 } \left\{\left[-\left(k \cdot l_{1}\right)\left(R \cdot l_{2}\right)-\left(k \cdot l_{2}\right)\left(R \cdot l_{1}\right)+\left(l_{1} \cdot l_{2}\right)(R \cdot k)\right]+\right.\right. \\
\left.\frac{-3}{k^{2}}\left[-2(k \cdot R)\left(k \cdot l_{1}\right)\left(k \cdot l_{2}\right)+\left(l_{1} \cdot l_{2}\right)(R \cdot k) k^{2}\right]\right\} \\
\left.+B_{1} B_{2}[2(R \cdot k)]\right]
\end{array}
$$

$$
\tilde{\Omega}_{F 3}=\Omega_{3}\left[A_{1} B_{2}\left[\left(l_{1} \cdot R\right)-3 \frac{\left(k \cdot l_{1}\right)(k \cdot R)}{k^{2}}\right]+A_{2} B_{1}\left[\left(l_{2} \cdot R\right)-3 \frac{\left(k \cdot l_{2}\right)(k \cdot R)}{k^{2}}\right]\right]
$$

$$
\begin{aligned}
& R^{\mu}=2 l^{\mu}-k^{\mu} \\
& l_{1}{ }^{\mu}=l^{\mu} \\
& l_{2}{ }^{\mu}=l^{\mu}-k^{\mu} \\
& \Omega_{1}^{\prime}=\Omega_{1}+\chi_{1} \\
& \Omega_{1}=\frac{A_{2}+A_{1}}{2} \\
& \Omega_{2}=\frac{1}{l_{2}^{2}-l_{1}^{2}} \frac{A_{2}-A_{1}}{2} \\
& \Omega_{3}=-\frac{1}{l_{2}^{2}-l_{1}^{2}}\left(B_{2}-B_{1}\right) \\
& \chi_{1}=\frac{A_{2}-A_{1}}{2} \frac{l_{2}{ }^{4}-l_{1}{ }^{4}}{\left(l_{2}{ }^{2}-l_{1}{ }^{2}\right)^{2}+\left[M_{2}^{2}+M_{1}^{2}\right]^{2}} \\
& \chi_{2}=-\frac{A_{2}-A_{1}}{2} \frac{l_{2}{ }^{2}+l_{1}{ }^{2}}{\left(l_{2}{ }^{2}-l_{1}{ }^{2}\right)^{2}+\left[M_{2}^{2}+M_{1}^{2}\right]^{2}} \\
& M=\frac{B}{A}
\end{aligned}
$$


- formula for $\mathcal{F}$ :

$$
\begin{gathered}
\mathcal{F}=i \frac{1}{2 k^{2}} g^{2} \int \frac{d^{3} l}{(2 \pi)^{3}} \frac{1}{A_{1}^{2} l_{1}^{2}-B_{1}^{2}} \frac{1}{A_{2}^{2} l_{2}^{2}-B_{2}^{2}}(\operatorname{tr} I)\left[\tilde{\Omega}_{\mathcal{F} 1}+\tilde{\Omega}_{\mathcal{F} 2}+\tilde{\chi}_{\mathcal{F} 2}+\tilde{\Omega}_{\mathcal{F} 3}\right] \\
\tilde{\Omega}_{\mathcal{F} 1}=2 \Omega_{1}^{\prime}\left[A_{1} B_{2} l_{1} \cdot k-A_{2} B_{1} l_{2} \cdot k\right] \\
\tilde{\Omega}_{\mathcal{F} 2}=\Omega_{2}\left\{A_{1} B_{2}\left[R^{2}\left(k \cdot l_{1}\right)-\left(R \cdot l_{1}\right)(R \cdot k)\right]-A_{2} B_{1}\left[R^{2}\left(k \cdot l_{2}\right)-\left(R \cdot l_{2}\right)(R \cdot k)\right]\right\} \\
\tilde{\chi}_{\mathcal{F} 2}=\chi_{2}\left\{A_{1} B_{2}\left[-(R \cdot k)\left(k \cdot l_{1}\right)+\left(R \cdot l_{1}\right) k^{2}\right]-A_{2} B_{1}\left[-(R \cdot k)\left(k \cdot l_{2}\right)+\left(R \cdot l_{2}\right) k^{2}\right]\right\} \\
\tilde{\Omega}_{\mathcal{F} 3}=\Omega_{3} A_{1} A_{2}\left[\left(k \cdot l_{1}\right)\left(R \cdot l_{2}\right)-\left(k \cdot l_{2}\right)\left(R \cdot l_{1}\right)\right]
\end{gathered}
$$

with

$$
\begin{aligned}
& R^{\mu}=2 l^{\mu}-k^{\mu} \\
& l_{1}{ }^{\mu}=l^{\mu} \\
& l_{2}{ }^{\mu}=l^{\mu}-k^{\mu} \\
& \Omega_{1}^{\prime}=\Omega_{1}+\chi_{1} \\
& \Omega_{1}=\frac{A_{2}+A_{1}}{2} \\
& \Omega_{2}=\frac{1}{l_{2}^{2}-l_{1}^{2}} \frac{A_{2}-A_{1}}{2} \\
& \Omega_{3}=-\frac{1}{l_{2}^{2}-l_{1}^{2}}\left(B_{2}-B_{1}\right) \\
& \chi_{1}=\frac{A_{2}-A_{1}}{2} \frac{l_{2}{ }^{4}-l_{1}{ }^{4}}{\left(l_{2}{ }^{2}-l_{1}^{2}\right)^{2}+\left[M_{2}^{2}+M_{1}^{2}\right]^{2}} \\
& \chi_{2}=-\frac{A_{2}-A_{1}}{2} \frac{l_{2}^{2}+l_{1}^{2}}{\left(l_{2}^{2}-l_{1}^{2}\right)^{2}+\left[M_{2}^{2}+M_{1}^{2}\right]^{2}} \\
& M=\frac{B}{A}
\end{aligned}
$$




\section{BIBLIOGRAPHY}

[1] R. Rivers, Path Integral Methods In Quantum Field Theory. Cambridge University Press, 1987.

[2] J. Negele and H. Orland, Quantum Many-Particle Systems. Addison-Wesley, 1988.

[3] B. Hatfield, Quantum Field Theory of Point Particles and Strings. Addison-Wesley, 1992.

[4] A. Zee, Quantum Field Theory in a Nutshell. Princeton University Press, 2003.

[5] M. E. Peskin and D. V. Schroeder, An Introduction to Quantum Field Theory. AddisonWesley, 1995.

[6] J. Kapusta and C. Gale, Finite Temperature Field Theory: Principles and Applications. Cambridge University Press, 2006.

[7] M. L. Bellac, Thermal Field Theory. Cambridge University Press, 1996.

[8] R. Shrock, "Some recent results on models of dynamical electroweak symmetry breaking," Arxiv preprint hep-ph/0703050, 2007.

[9] S. Weinberg, "Implications of dynamical symmetry breaking," Phys. Rev. D, vol. 13, pp. 974-996, Feb 1976.

[10] S. Dimopoulos and L. Susskind, "Mass without scalars," Nucl. Phys. B, vol. 155, no. 1, pp. $237-252,1979$.

[11] B. Holdom, "Raising the sideways scale," Phys. Rev. D, vol. 24, no. 5, pp. 1441-1444, 1981.

[12] C. T. Hill and E. H. Simmons, "Strong dynamics and electroweak symmetry breaking," Phys. Rept., vol. 381, no. 4-6, pp. 235 - 402, 2003.

[13] E. S. Swanson, "QCD beyond the standard model," Acta Phys. Polon. Supp., vol. 3, pp. 991-995, 2010.

[14] V. Koch, "Aspects of chiral symmetry," Int. J. Mod. Phys., vol. E6, pp. 203-250, 1997. 
[15] J. Donoghue, E. Golowich, and B. Holstein, Dynamics of the Standard Model, vol. 2. Cambridge University Press, 1994.

[16] T. E. O. Ericson, B. Loiseau, and A. W. Thomas, "Determination of the pion-nucleon coupling constant and scattering lengths," Phys. Rev. C, vol. 66, p. 014005, Jul 2002.

[17] L. Kadanoff, "More is the same; phase transitions and mean field theories," Journal of Statistical Physics, vol. 137, pp. 777-797, 2009. 10.1007/s10955-009-9814-1.

[18] S. P. Klevansky, "The nambu-jona-lasinio model of quantum chromodynamics," Rev. Mod. Phys., vol. 64, pp. 649-708, Jul 1992.

[19] P. M. Lo and E. S. Swanson, "Confinement models at finite temperature and density," Phys. Rev. D, vol. 81, p. 034030, Feb 2010.

[20] E. V. Shuryak, "Quantum Chromodynamics and the Theory of Superdense Matter," Phys. Rept., vol. 61, pp. 71-158, 1980.

[21] A. Mocsy and P. Petreczky, "Quarkonium melting above deconfinement," J.Phys.G, vol. G35, p. 104154, 2008.

[22] N. H. Christ and T. D. Lee, "Operator ordering and feynman rules in gauge theories," Phys. Rev. D, vol. 22, pp. 939-958, Aug 1980.

[23] A. P. Szczepaniak and E. S. Swanson, "Coulomb gauge QCD, confinement, and the constituent representation," Phys. Rev., vol. D65, p. 025012, 2002.

[24] R. Mattuck, A Guide to Feynman Diagrams in the Many Body Problem (Second Edition). Dover Publications (New York), 1992.

[25] V. Miransky, Dynamical Symmetry Breaking in Quantum Field Theories. World Scientific, 1993.

[26] A. Davis and A. Matheson, "Chiral symmetry breaking at finite temperature in Coulomb gauge QCD," Nucl. Phys., vol. B246, p. 203, 1984.

[27] R. Alkofer, P. A. Amundsen, and K. Langfeld, "Chiral symmetry breaking and pion properties at finite temperatures," Z. Phys., vol. C42, pp. 199-208, 1989.

[28] A. Le Yaouanc, L. Oliver, O. Pene, J. Raynal, M. Jarfi, et al., "Introducing thermal excitations for color potentials," Phys. Rev., vol. D39, p. 924, 1989.

[29] M. Gell-Mann and K. A. Brueckner, "Correlation Energy of an Electron Gas at High Density," Phys. Rev., vol. 106, pp. 364-368, 1957.

[30] M. Stephanov, "QCD phase diagram: An Overview," PoS, vol. LAT2006, p. 024, 2006. 
[31] A. Andronic, D. Blaschke, P. Braun-Munzinger, J. Cleymans, K. Fukushima, L. McLerran, H. Oeschler, R. Pisarski, K. Redlich, C. Sasaki, H. Satz, and J. Stachel, "Hadron production in ultra-relativistic nuclear collisions: Quarkyonic matter and a triple point in the phase diagram of qcd," Nucl. Phys. A, vol. 837, no. 1-2, pp. 65-86, 2010.

[32] G. Odyniec, "RHIC beam energy scan program-experimental approach to the QCD phase diagram," J.Phys.G, vol. G37, p. 094028, 2010.

[33] J. M. Heuser, "The Compressed Baryonic Matter Experiment at FAIR: Progress with feasibility studies and detector developments," Nucl.Phys., vol. A830, pp. 563C-566C, 2009.

[34] P. Petreczky, "Lattice QCD at finite temperature," Nucl.Phys., vol. A785, pp. 10-17, 2007.

[35] J. Wambach, "Recent theoretical developments in the qcd phase diagram," Arxiv preprint arXiv:1101.4760, 2011.

[36] M. A. Stephanov, K. Rajagopal, and E. V. Shuryak, "Signatures of the tricritical point in QCD," Phys. Rev.Lett., vol. 81, pp. 4816-4819, 1998.

[37] L. McLerran and R. D. Pisarski, "Phases of cold, dense quarks at large N(c)," Nucl. Phys., vol. A796, pp. 83-100, 2007.

[38] M. Kloker, The QCD Quark Propagator in Coulomb Gauge and some Implications for Hadronic Physics. PhD thesis, Universitat Tubingen, 2007.

[39] P. M. Lo and E. S. Swanson, "Parity symmetry in qed3," Phys. Rev. D, vol. 83, p. 065006, Mar 2011.

[40] R. D. Pisarski, "Chiral Symmetry Breaking in Three-Dimensional Electrodynamics," Phys. Rev., vol. D29, p. 2423, 1984.

[41] T. W. Appelquist, M. J. Bowick, D. Karabali, and L. Wijewardhana, "Spontaneous Chiral Symmetry Breaking in Three-Dimensional QED," Phys. Rev., vol. D33, p. 3704, 1986.

[42] S. R. Coleman and B. R. Hill, "No More Corrections to the Topological Mass Term in QED in Three-Dimensions," Phys. Lett., vol. B159, p. 184, 1985.

[43] S. Deser, R. Jackiw, and S. Templeton, "Topologically Massive Gauge Theories," Annals Phys., vol. 140, pp. 372-411, 1982.

[44] P. Maris, "The Influence of the full vertex and vacuum polarization on the fermion propagator in QED in three-dimensions," Phys. Rev., vol. D54, pp. 4049-4058, 1996.

[45] H. Politzer, "Effective Quark Masses in the Chiral Limit," Nucl. Phys., vol. B117, p. 397, 1976. 
[46] C. Fischer, R. Alkofer, T. Dahm, and P. Maris, "Dynamical chiral symmetry breaking in unquenched QED(3)," Phys. Rev., vol. D70, p. 073007, 2004.

[47] D. Curtis and M. Pennington, "Truncating the Schwinger-Dyson equations: How multiplicative renormalizability and the Ward identity restrict the three point vertex in QED," Phys. Rev., vol. D42, pp. 4165-4169, 1990.

[48] A. Bashir, A. Raya, S. Sanchez-Madrigal, and C. Roberts, "Gauge invariance of a critical number of flavours in QED3," Few Body Syst., vol. 46, pp. 229-237, 2009.

[49] A. Bashir, A. Raya, I. Cloet, and C. Roberts, "Regarding confinement and dynamical chiral symmetry breaking in QED3," Phys. Rev., vol. C78, p. 055201, 2008.

[50] M. Pennington and D. Walsh, "Masses from nothing: A Nonperturbative study of QED in three-dimensions," Phys.Lett., vol. B253, pp. 246-251, 1991.

[51] C. J. Burden, J. Praschifka, and C. D. Roberts, "Photon polarization tensor in threedimensional quantum electrodynamics," Phys. Rev., vol. D46, pp. 2695-2702, 1992.

[52] C. Vafa and E. Witten, "Parity Conservation in QCD," Phys. Rev. Lett., vol. 53, p. 535, 1984.

[53] P. M. Lo and E. S. Swanson, "Endemic infrared divergences in qed3 at finite temperature," Physics Letters B, vol. 697, no. 2, pp. 164 - 167, 2011.

[54] G. Triantaphyllou, "Gap equation in a finite temperature gauge theory beyond the bare vertex approximation," JHEP, vol. 9903, p. 020, 1999.

[55] D. Lee and G. Metikas, "Infrared divergence in $\operatorname{QED}(3)$ at finite temperature," Int. J. Mod. Phys., vol. A14, pp. 2921-2948, 1999.

[56] N. Dorey and N. Mavromatos, "QED in three-dimension and two-dimensional superconductivity without parity violation," Nucl. Phys., vol. B386, pp. 614-682, 1992.

[57] I. Aitchison and M. Klein-Kreisler, "Effect of wave function renormalization in N flavor QED in three-dimensions at finite temperature," Phys. Rev., vol. D50, pp. 1068-1076, 1994.

[58] M. He, H.-T. Feng, W.-M. Sun, and H.-S. Zong, "Phase Structure of QED3 at Finite Chemical Potential and Temperature," Modern Physics Letters A, vol. 22, pp. 449-456, 2007.

[59] J.-P. Blaizot, E. Iancu, and R. R. Parwani, "On the screening of static electromagnetic fields in hot QED plasmas," Phys. Rev., vol. D52, pp. 2543-2562, 1995.

[60] J. I. Kapusta, "Screening of static qed electric fields in hot qcd," Phys. Rev. D, vol. 46, pp. 4749-4753, Nov 1992. 
[61] E. S. Swanson, "A Primer on Functional Methods and the Schwinger-Dyson Equations," AIP Conf. Proc., vol. 1296, pp. 75-121, 2010.

[62] L. Moyaerts, A Numerical Study of Quantum Forces : Casimir Effect, Vortices and Coulomb Gauge Yang-Mills Theory. PhD thesis, Universitat Tubingen, 2004.

[63] V. N. Gribov, "Quantization of non-abelian gauge theories," Nucl. Phys. B, vol. 139, no. 1-2, pp. 1 - 19, 1978.

[64] D. Zwanziger, "Fundamental modular region, Boltzmann factor, and area law in lattice theory," Nucl. Phys. Proc. Suppl., vol. 34, pp. 198-200, 1994.

[65] J. Greensite, S. Olejnik, and D. Zwanziger, "Coulomb energy, remnant symmetry, and the phases of nonAbelian gauge theories," Phys. Rev., vol. D69, p. 074506, 2004.

[66] E. S. Swanson, "Aspects of confinement: A Brief review," AIP Conf. Proc., vol. 717, pp. 636-645, 2004.

[67] R. Alkofer and L. von Smekal, "The infrared behavior of QCD Green's functions: Confinement, dynamical symmetry breaking, and hadrons as relativistic bound states," Phys. Rept., vol. 353, p. 281, 2001. 